\title{
Substituent Effect Induces Emission Modulation of Stilbene Photoswitches by Spatial Tuning the N/B Electronic Constraints
}

\author{
Guangqian Ji, Nan Wang, Xiaodong Yin, and Pangkuan Chen* \\ Beijing Key Laboratory of Photoelectronic/Electrophotonic Conversion Materials, Key Laboratory of \\ Cluster Science of the Ministry of Education, School of Chemistry and Chemical Engineering, Beijing \\ Institute of Technology of China, Beijing, 102488, China \\ *pangkuan@bit.edu.cn
}

\section{Experimental Section}

Materials and General Methods: All reactions were carried out under nitrogen atmosphere in oven-dried glassware unless otherwise mentioned. Bromobenzylbromide, triphenylphosphine, $n$-butyllithium, $t$-butyllithium, 2 bromobenzaldehyde, diaylamines, $\mathrm{Pd}_{2}(\mathrm{dba})_{3}$, potassium $t$-butoxide, tri- $t$ butylphosphine, 2-bromo-N,N-dimethylaniline, 2-bromoaniline and dimesitylboron fluoride were purchased from Energy Chemical. Tetrahydrofuran, dichloromethane, petroleum ether, methanol and ethyl acetate were purchased from Sinopharm Chemical Reagent Co., Ltd. The chemicals were used without further purification unless otherwise noted. Anhydrous solvents were distilled from commercial solvent with sodium/benzopheneone.

All commercial chemicals were used without further purification. Compounds $\mathbf{a}^{1}, \mathbf{b}^{2}$, $\mathbf{d}^{3}, \mathbf{g}^{4}, \mathbf{h}^{5}, \mathbf{i}^{6}, \mathbf{j}^{4}, \mathbf{3}^{5}$ were prepared using the similar procedures previously described. Those reactions at elevated temperature were performed using oil bath unless otherwise mentioned. ${ }^{1} \mathrm{H}$ NMR spectra were recorded on a $400 \mathrm{MHz}$ Bruker spectrometer. ${ }^{13} \mathrm{C}$ NMR spectra were recorded on the 101 Bruker spectrometers. ${ }^{11} \mathrm{~B}$ NMR spectra were recorded on a $128 \mathrm{MHz}$ Bruker spectrometer. ${ }^{11} \mathrm{~B}$ NMR spectra were acquired with boron-free quartz NMR tubes and the spectra were referenced externally to $\mathrm{BF}_{3} \cdot \mathrm{Et}_{2} \mathrm{O}$ $(\delta=0)$. High resolution mass spectral data were obtained via ESI on an Agilent (Q-TOF $6520)$ analyzer.

UV-visible absorption spectra were recorded on a Cary 300 UV-Vis spectrophotometer. Luminescent spectra were recorded on an Edinburgh Instruments FLS980 or Lengguang Tech F97PrO spectrophotometer. Fluorescent quantum efficiencies were determined using a Hamamatsu C11347-11 Quantaurus-QY spectrometer.

Single crystals 1-4 were obtained by slow evaporation from solutions of $\mathrm{CH}_{2} \mathrm{Cl}_{2} / \mathrm{MeOH}$. X-ray analysis was obtained on a Bruker D8 X-ray single crystal Venture diffractometer. SAINT5.0 and SADABS programs are used for the reduction and absorption correction of crystal data. The resolution and refinement of the crystal 
structure are obtained on the SHELXTL-97 software. Using the direct or Patterson methods, all non-hydrogen source coordinates are obtained by using the differential Fourier method and the least square method. Then the geometric method and the difference value are used. The hydrogen atom coordinates were obtained by Fourier method, and the crystal structure was obtained. The CCDC numbers of 1947373 (1), 1935891 (2) and 1935892 (4) are deposited.

DFT calculations were performed with the Gaussian 09 program. Geometry optimizations and vertical excitations were calculated by means of hybrid density functional B3LYP and CAM-B3LYP with the basis set of 6-31G*. The input files of 2 , $\mathbf{3}$ and $\mathbf{4}$ were generated from the CIF files, and the input file of $\mathbf{1}$ and all the orbital representations were generated with Gaussview 5.0 (scaling radii of $75 \%$, isovalue $=$ 0.02). Excitation data were calculated using TD-DFT (B3LYP/6-311G**). The true minimum of resulting structures was confirmed to be stationary points through vibrational frequency analysis with absorbance of imaginary frequencies. 


\section{Synthetic Procedures}

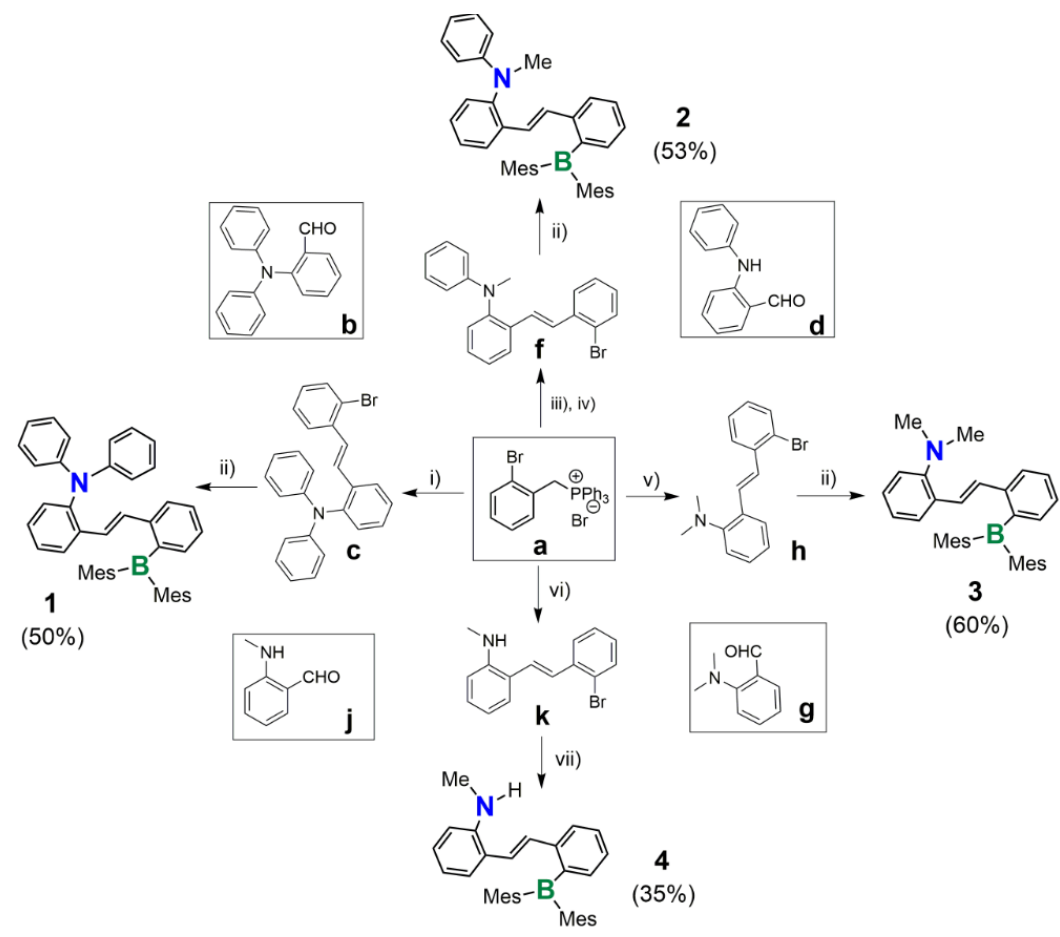

Reagents and conditions: i) $\mathbf{b}$ and $\mathrm{NaH}, \mathrm{THF}, 0{ }^{\circ} \mathrm{C}$ to rt. ii) $n$-BuLi, THF, $-78{ }^{\circ} \mathrm{C}$ for 2 h, then dimesitylboron fluoride (Mes2 $\mathbf{B F}$ ). iii) $\mathbf{d}$ and $\mathrm{NaH}$, THF, $0{ }^{\circ} \mathrm{C}$ to rt. iv) $\mathrm{K}_{2} \mathrm{CO}_{3}$, $\mathrm{CH}_{3} \mathrm{I}$, DMF, rt. v) $\mathbf{g}$ and $\mathrm{NaH}, \mathrm{THF}, 0{ }^{\circ} \mathrm{C}$ to rt. vi) $\mathbf{j}$ and $\mathrm{NaH}$, THF, $0{ }^{\circ} \mathrm{C}$ to rt. vii) $t$ BuLi, THF, $-78{ }^{\circ} \mathrm{C}$ for $2 \mathrm{~h}$, then $\mathbf{M e s}_{2} \mathbf{B F}$. The intermediates $\mathbf{a}, \mathbf{b}, \mathbf{d}, \mathbf{g}, \mathbf{h}, \mathbf{j}$ and compound 3 were prepared via the literature procedures (ref 1-6).

\subsection{Synthesis of a}
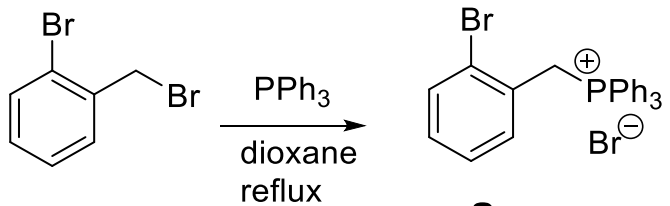

a

Compound a was synthesized via the previously reported procedure ${ }^{1}$.

\subsection{Synthesis of $b$}<smiles>O=Cc1ccccc1Br</smiles><smiles>c1ccc(Nc2ccccc2)cc1</smiles>

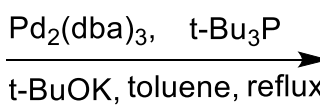<smiles>O=Cc1ccccc1N(c1ccccc1)c1ccccc1</smiles> 
Compound $\mathbf{b}$ was synthesized via the previously reported procedure ${ }^{2}$.

\subsection{Synthesis of $c$}

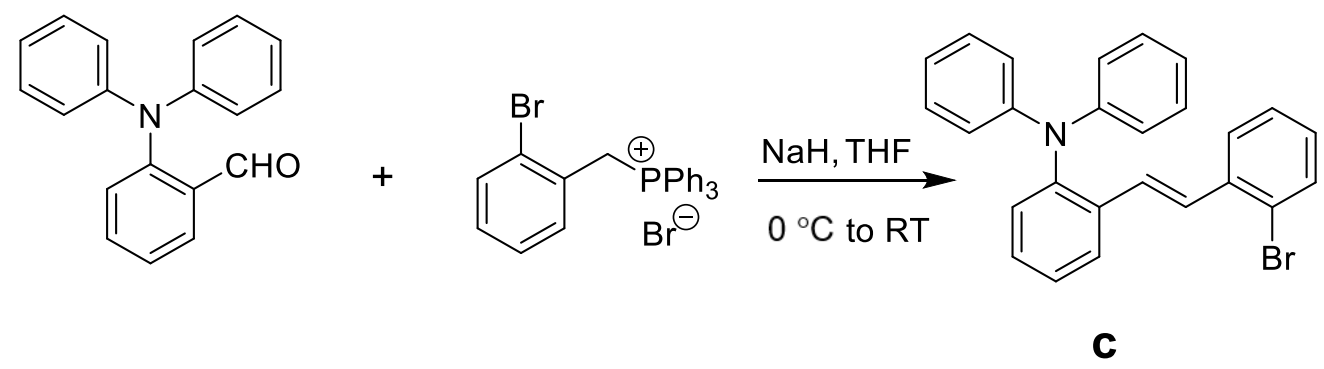

$\mathrm{NaH}$ (120 mg, $5.0 \mathrm{mmol})$ was added to a solution of $\mathbf{a}(1 \mathrm{~g}, 2.0 \mathrm{mmol})$ in anhydrous THF $(15 \mathrm{~mL})$ at $0{ }^{\circ} \mathrm{C}$. After $1 \mathrm{~h}, \mathbf{b}(273 \mathrm{mg}, 1.0 \mathrm{mmol})$ in anhydrous THF $(20 \mathrm{~mL})$ was added by syringe at $0{ }^{\circ} \mathrm{C}$. The reaction mixture was warmed up to room temperature and stirred overnight. The reaction was quenched with a saturated brine and the aqueous layer was extracted with $\mathrm{CH}_{2} \mathrm{Cl}_{2}(20 \mathrm{~mL} \times 3)$. The organic layer was combined and dried over anhydrous $\mathrm{Na}_{2} \mathrm{SO}_{4}$, filtered, and concentrated under reduced pressure. The crude product was purified by column chromatography on silica gel (petroleum ether: $\mathrm{CH}_{2} \mathrm{Cl}_{2}=4:$ 1) to afford compound $\mathbf{c}(362 \mathrm{mg})$ in $85 \%$ yield as a pale green oil. ${ }^{1} \mathrm{H}$ NMR $\left(400 \mathrm{MHz}, \mathrm{CDCl}_{3}\right) \delta 7.52(\mathrm{~d}, J=9.3 \mathrm{~Hz}, 1 \mathrm{H}), 7.25-7.18(\mathrm{~m}, 5 \mathrm{H}), 7.16(\mathrm{~s}, 1 \mathrm{H})$, $7.06-6.99(\mathrm{~m}, 7 \mathrm{H}), 6.92(\mathrm{t}, J=7.3 \mathrm{~Hz}, 3 \mathrm{H}), 6.88-6.84(\mathrm{~m}, 1 \mathrm{H}), 6.57$ (d, $J=12.1 \mathrm{~Hz}$, $1 \mathrm{H}), 6.39(\mathrm{~d}, J=12.1 \mathrm{~Hz}, 1 \mathrm{H}) .{ }^{13} \mathrm{C} \mathrm{NMR}\left(101 \mathrm{MHz}, \mathrm{CDCl}_{3}\right) \delta 148.0,146.0,137.7$, 134.6, 132.6, 131.3, 130.5, 129.5, 129.2, 129.1, 128.9, 128.9, 128.6, 126.9, 125.1, 124.0, 122.3, 121.9. HRMS (ESI-TOF) $\mathrm{m} / \mathrm{z}$ : $[\mathrm{M}+\mathrm{H}]^{+}$calculated for $\mathrm{C}_{26} \mathrm{H}_{21} \mathrm{BrN} 426.0857$, found 426.0852 .

\subsection{Synthesis of 1}<smiles>Brc1ccccc1/C=C/c1ccccc1N(c1ccccc1)c1ccccc1</smiles>

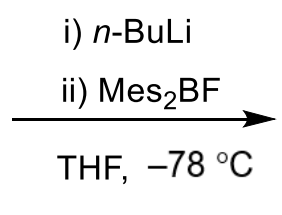<smiles>CC(C)(C)c1ccccc1/C=C/c1ccccc1N(c1ccccc1)c1ccccc1</smiles>

A solution of $n$-BuLi in hexane $(1 \mathrm{~mL}, 1.6 \mathrm{M}, 1.6 \mathrm{mmol})$ was added dropwise by syringe at $-78{ }^{\circ} \mathrm{C}$ to a solution of $\mathbf{c}(213 \mathrm{mg}, 0.5 \mathrm{mmol})$ in anhydrous THF $(10 \mathrm{~mL})$. The mixture was stirred at the same temperature for $2 \mathrm{~h}$. A solution of dimesitylboron fluoride $(161 \mathrm{mg}, 0.6 \mathrm{mmol})$ in anhydrous THF $(5 \mathrm{~mL})$ was added to the reaction mixture using a syringe. The reaction mixture was warmed up to room temperature and stirred overnight. The reaction was quenched with a saturated brine and the aqueous layer was extracted with $\mathrm{CH}_{2} \mathrm{Cl}_{2}(20 \mathrm{~mL} \times 3)$. The organic layer was combined and dried over anhydrous $\mathrm{Na}_{2} \mathrm{SO}_{4}$, filtered, and concentrated under reduced pressure. The crude product was purified by column chromatography on silica gel (petroleum ether: $\left.\mathrm{CH}_{2} \mathrm{Cl}_{2}=8: 1\right)$ to afford compound $1(149 \mathrm{mg})$ in $50 \%$ yield as a pale yellow solid. $\mathrm{m}$. p. $193-195{ }^{\circ} \mathrm{C} .{ }^{1} \mathrm{H}$ NMR $\left(400 \mathrm{MHz}, \mathrm{C}_{6} \mathrm{D}_{6}\right) \delta 7.52(\mathrm{~d}, J=7.7 \mathrm{~Hz}, 1 \mathrm{H}), 7.44(\mathrm{~d}, J=7.4$ 
$\mathrm{Hz}, 1 \mathrm{H}), 7.29(\mathrm{~d}, J=7.1 \mathrm{~Hz}, 1 \mathrm{H}), 6.93(\mathrm{t}, J=7.0 \mathrm{~Hz}, 2 \mathrm{H}), 6.98(\mathrm{~d}, J=8.6 \mathrm{~Hz}, 4 \mathrm{H})$, 6.95-6.89 (m, 6H), $6.84(\mathrm{t}, J=7.0 \mathrm{~Hz}, 1 \mathrm{H}), 6.76-6.73(\mathrm{~m}, 2 \mathrm{H}), 6.63(\mathrm{~d}, J=12 \mathrm{~Hz}$, $\mathrm{HC}=\mathrm{CH}, 1 \mathrm{H}), 6.44(\mathrm{~d}, J=12 \mathrm{~Hz}, \mathrm{HC}=\mathrm{CH}, 1 \mathrm{H}), 6.30(\mathrm{~s}, 4 \mathrm{H}), 2.14(\mathrm{~s}, 12 \mathrm{H}), 2.12(\mathrm{~s}$, $6 \mathrm{H}) .{ }^{13} \mathrm{C}$ NMR (101 MHz, $\left.\mathrm{C}_{6} \mathrm{D}_{6}\right) \delta 148.3,148.1,146.3,143.3,141.6,140.2,138.9$, $135.8,135.7,135.6,135.0,131.4,131.3,131.3,131.0,130.2,129.5,129.3,129.1,129.0$, 128.7, 128.6, 128.5, 127.1, 126.9, 126.0, 125.4,125.3, 124.8, 122.4, 122.3, 121.7, 23.2, 21.0. ${ }^{11} \mathrm{~B}$ NMR (225 MHz, $\left.\mathrm{C}_{6} \mathrm{D}_{6}\right) \delta 77 \mathrm{ppm}$. HRMS MALDI-MS (pos.) m/z: calculated for $\mathrm{C}_{44} \mathrm{H}_{42} \mathrm{BN}\left[\mathrm{M}^{+}\right]$595.3410, found 595.3396.

\subsection{Synthesis of $d$}<smiles>Nc1ccccc1</smiles>

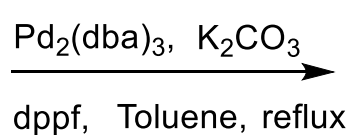<smiles>Cc1ccccc1Nc1ccccc1</smiles>

d

Compound $\mathbf{d}$ was synthesized via the previously reported procedure ${ }^{3}$.

\subsection{Synthesis of $f$}

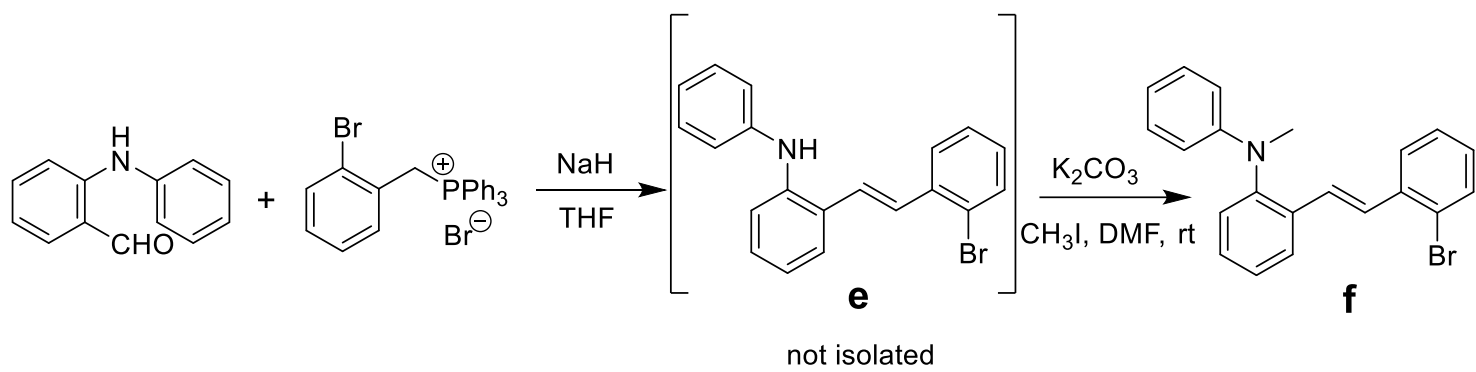

$\mathrm{NaH}$ (120 mg, $5.0 \mathrm{mmol})$ was added to a solution of $\mathbf{a}(1 \mathrm{~g}, 2.0 \mathrm{mmol})$ in anhydrous THF $(15 \mathrm{~mL})$ at $0{ }^{\circ} \mathrm{C}$. After $1 \mathrm{~h}, \mathbf{d}(197 \mathrm{mg}, 1.0 \mathrm{mmol})$ in anhydrous THF (20 mL) was added by syringe at $0{ }^{\circ} \mathrm{C}$. The reaction mixture was warmed up to room temperature and stirred overnight. The reaction mixture was warmed up to room temperature and stirred overnight. The reaction was quenched with a saturated brine and the aqueous layer was extracted with $\mathrm{CH}_{2} \mathrm{Cl}_{2}(20 \mathrm{~mL} \times 3)$. The organic layer was combined and dried over anhydrous $\mathrm{Na}_{2} \mathrm{SO}_{4}$, filtered, and concentrated under reduced pressure. Without further isolation, a solution of resulting compound e in DMF (10 mL) was added to anhydrous $\mathrm{K}_{2} \mathrm{CO}_{3}(690 \mathrm{mg}, 5.0 \mathrm{mmol})$ at room temperature. To the resulting suspension was added $1.2 \mathrm{~mL}$ of $\mathrm{CH}_{3} \mathrm{I}(5.0 \mathrm{mmol})$, and the mixture was heated to $100{ }^{\circ} \mathrm{C}$ and kept stirring until completion of the reaction as determined by TLC. After $8 \mathrm{~h}$, the reaction was quenched with water $(7 \mathrm{~mL})$, and then extracted with $\mathrm{CH}_{2} \mathrm{Cl}_{2}(3 \times$ $10 \mathrm{~mL}$ ). The organic phases were dried over anhydrous $\mathrm{Na}_{2} \mathrm{SO}_{4}$, and the solvent was evaporated under reduced. The resulting mixture was purified by column chromatography on silica gel (petroleum ether: $\mathrm{CH}_{2} \mathrm{Cl}_{2}=4: 1$ ) to afford compound $\mathbf{f}$ ( $218 \mathrm{mg}, 60 \%$ overall yield in two steps) as a pale yellow oil. ${ }^{1} \mathrm{H}$ NMR $(400 \mathrm{MHz}$, $\left.\mathrm{CDCl}_{3}\right) \delta 7.82(\mathrm{~d}, J=9.4 \mathrm{~Hz}, 1 \mathrm{H}), 7.52(\mathrm{~d}, J=8.0 \mathrm{~Hz}, 1 \mathrm{H}), 7.45(\mathrm{~d}, J=16.3 \mathrm{~Hz}, 1 \mathrm{H})$, $7.40(\mathrm{~d}, J=7.9 \mathrm{~Hz}, 1 \mathrm{H}), 7.30(\mathrm{ddd}, J=7.1,4.5,1.9 \mathrm{~Hz}, 2 \mathrm{H}), 7.18(\mathrm{t}, J=8.0 \mathrm{~Hz}, 4 \mathrm{H})$, 
$7.12(\mathrm{~d}, J=16.3 \mathrm{~Hz}, 1 \mathrm{H}), 7.04(\mathrm{t}, J=8 \mathrm{~Hz}, 1 \mathrm{H}), 6.75(\mathrm{t}, J=6.8 \mathrm{~Hz}, 1 \mathrm{H}), 6.66(\mathrm{~d}, J=$ $8.7 \mathrm{~Hz}, 2 \mathrm{H}), 3.22(\mathrm{~s}, 3 \mathrm{H}) .{ }^{13} \mathrm{C} \mathrm{NMR}\left(101 \mathrm{MHz}, \mathrm{CDCl}_{3}\right) \delta 149.6,147.1,137.4,135.4$, 133.0, 129.6, 129.0, 128.8, 128.7, 128.4, 127.7, 127.8, 127.0, 126.9, 126.6, 124.2, 117.7, 114.1, 40.1. HRMS (ESI-TOF) m/z: $[\mathrm{M}+\mathrm{H}]^{+}$calculated for $\mathrm{C}_{21} \mathrm{H}_{29} \mathrm{BrN} 364.0701$, found 364.0694 .

\subsection{Synthesis of 2}

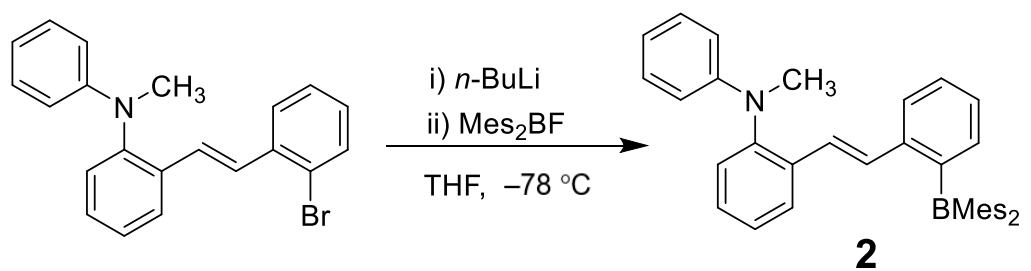

A solution of $n$-BuLi in hexane $(1 \mathrm{~mL}, 1.6 \mathrm{M}, 1.6 \mathrm{mmol})$ was added dropwise by syringe at $-78{ }^{\circ} \mathrm{C}$ to a solution of $\mathbf{f}(182 \mathrm{mg}, 0.5 \mathrm{mmol})$ in anhydrous THF $(10 \mathrm{~mL})$. The mixture was stirred at the same temperature for $2 \mathrm{~h}$. A solution of dimesitylboron fluoride (161 mg, $0.6 \mathrm{mmol})$ in anhydrous THF $(5 \mathrm{~mL})$ was added to the reaction mixture using a syringe. The reaction mixture was warmed up to room temperature and stirred overnight. The reaction was quenched with a saturated brine and the aqueous layer was extracted with $\mathrm{CH}_{2} \mathrm{Cl}_{2}(20 \mathrm{~mL} \times 3)$. The organic layer was combined and dried over anhydrous $\mathrm{Na}_{2} \mathrm{SO}_{4}$, filtered, and concentrated under reduced pressure. The resulting mixture was purified by column chromatography on silica gel (petroleum ether: $\left.\mathrm{CH}_{2} \mathrm{Cl}_{2}=8: 1\right)$ to afford compound $2(141 \mathrm{mg})$ in $53 \%$ yield as a yellow green solid. m. p. $177-180{ }^{\circ} \mathrm{C} .{ }^{1} \mathrm{H}$ NMR $\left(400 \mathrm{MHz}, \mathrm{C}_{6} \mathrm{D}_{6}\right) \delta 7.54(\mathrm{~d}, J=16 \mathrm{~Hz}, \mathrm{HC}=\mathrm{CH}, 1 \mathrm{H})$, $7.45(\mathrm{t}, J=7.1 \mathrm{~Hz}, 2 \mathrm{H}), 7.23(\mathrm{~d}, J=16 \mathrm{~Hz}, \mathrm{HC}=\mathrm{CH}, 1 \mathrm{H}), 7.14-7.07$ (m, 2H), 7.07$6.96(\mathrm{~m}, 5 \mathrm{H}), 6.91(\mathrm{~d}, J=6.6 \mathrm{~Hz}, 1 \mathrm{H}), 6.76(\mathrm{~d}, J=9.3 \mathrm{~Hz}, 5 \mathrm{H}), 6.63(\mathrm{~d}, J=8.7 \mathrm{~Hz}$, $2 \mathrm{H}), 2.87(\mathrm{~s}, 3 \mathrm{H}), 2.16(\mathrm{~s}, 6 \mathrm{H}), 2.13(\mathrm{~s}, 12 \mathrm{H}) .{ }^{13} \mathrm{C} \mathrm{NMR}\left(101 \mathrm{MHz}, \mathrm{C}_{6} \mathrm{D}_{6}\right) \delta 149.7$, $147.3,146.9,143.8,142.7,140.3,139.0,136.3,135.5,131.5,131.4,129.0,128.8,128.7$, $126.9,125.8,125.3,125.0,117.8,114.3,39.5,23.1,21.0 .{ }^{11} \mathrm{~B}$ NMR (225 MHz, C6 6 ) $\delta 78 \mathrm{ppm}$. HRMS MALDI-MS (pos.) m/z: calculated for $\mathrm{C}_{39} \mathrm{H}_{40} \mathrm{BN}\left[\mathrm{M}^{+}\right] 533.3254$, found 533.3244 .

\subsection{Synthesis of $\mathrm{g}$}

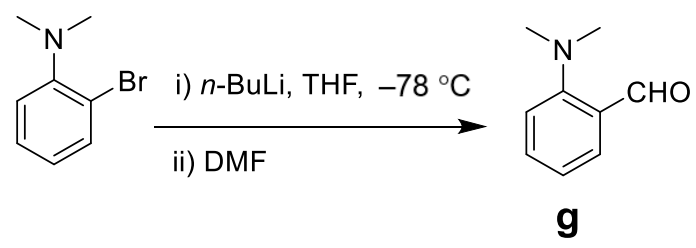

Compound $\mathbf{g}$ was synthesized via the previously reported procedure ${ }^{4}$. 


\subsection{Synthesis of $h$}

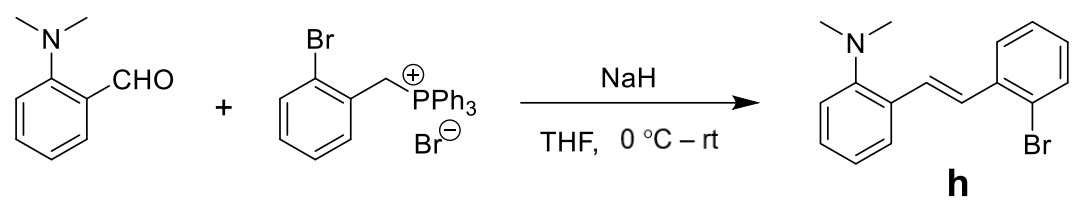

Compound $\mathbf{h}$ was synthesized via the previously reported procedure ${ }^{5}$.

\subsection{Synthesis of 3}<smiles>CN(C)c1ccccc1/C=C/c1ccccc1Br</smiles>

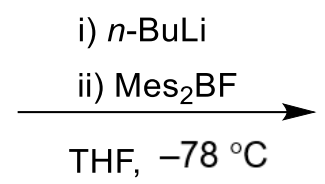<smiles>CN(C)c1ccccc1/C=C/c1ccccc1S(C)(=O)=O</smiles>

3

A solution of $n$-BuLi in hexane $(1 \mathrm{~mL}, 1.6 \mathrm{M}, 1.6 \mathrm{mmol})$ was added dropwise by syringe at $-78{ }^{\circ} \mathrm{C}$ to a solution of $\mathbf{h}(151 \mathrm{mg}, 0.5 \mathrm{mmol})$ in anhydrous THF $(10 \mathrm{~mL})$. The mixture was stirred at the same temperature for $2 \mathrm{~h}$. A solution of dimesitylboron fluoride $(161 \mathrm{mg}, 0.6 \mathrm{mmol})$ in anhydrous THF $(5 \mathrm{~mL})$ was added to the reaction mixture using a syringe. The reaction mixture was warmed up to room temperature and stirred overnight. The reaction mixture was warmed up to room temperature and stirred overnight. The reaction was quenched with a saturated brine and the aqueous layer was extracted with $\mathrm{CH}_{2} \mathrm{Cl}_{2}(20 \mathrm{~mL} \times 3)$. The organic layer was combined and dried over anhydrous $\mathrm{Na}_{2} \mathrm{SO}_{4}$, filtered, and concentrated under reduced pressure. The resulting mixture was purified by column chromatography on silica gel (petroleum ether: $\mathrm{CH}_{2} \mathrm{Cl}_{2}$ $=8: 1)$ to afford compound $\mathbf{3}(141 \mathrm{mg})$ in $60 \%$ yield as a yellow green solid.

The ${ }^{1} \mathrm{H}, \mathrm{C}$ NMR and HRMS spectroscopic data are consistent with those of the previously reported literature compound ${ }^{5}{ }^{11} \mathrm{~B}$ NMR $\left(225 \mathrm{MHz}, \mathrm{C}_{6} \mathrm{D}_{6}\right) \delta 50 \mathrm{ppm}$.

\subsection{Synthesis of $i$}<smiles>Nc1ccccc1Br</smiles><smiles>CCCCC(C)=O</smiles><smiles>CNc1ccc(I)cc1Br</smiles>

Compound $\mathbf{i}$ was synthesized via the previously reported procedure ${ }^{6}$. 


\subsection{Synthesis of $\mathbf{j}$}

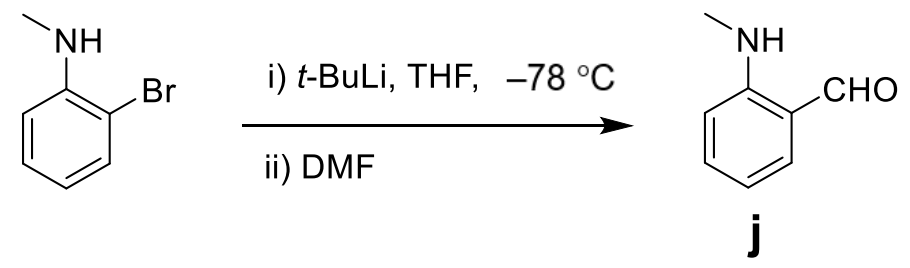

Compound $\mathbf{j}$ was synthesized via the previously reported procedure ${ }^{4}$.

\subsection{Synthesis of $k$}

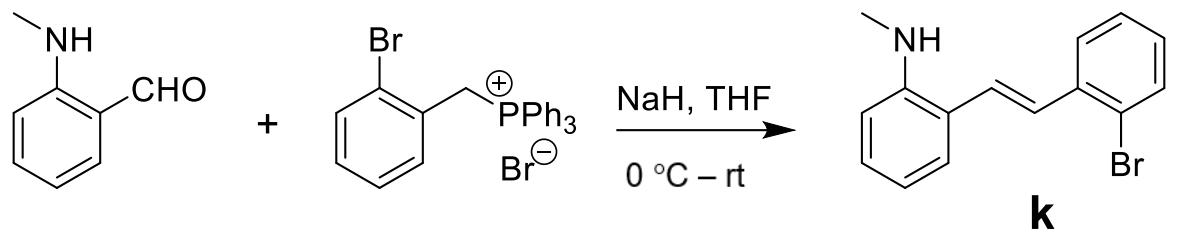

$\mathrm{NaH}(120 \mathrm{mg}, 5.0 \mathrm{mmol})$ was added to a solution of $\mathbf{a}(1 \mathrm{~g}, 2.0 \mathrm{mmol})$ in anhydrous THF $(15 \mathrm{~mL})$ at $0{ }^{\circ} \mathrm{C}$. After $1 \mathrm{~h}, \mathbf{i}(135 \mathrm{mg}, 1.0 \mathrm{mmol})$ in anhydrous THF $(20 \mathrm{~mL})$ was added by syringe at $0{ }^{\circ} \mathrm{C}$. The reaction mixture was warmed to room temperature and stirred overnight. The reaction mixture was warmed up to room temperature and stirred overnight. The reaction was quenched with a saturated brine and the aqueous layer was extracted with $\mathrm{CH}_{2} \mathrm{Cl}_{2}(20 \mathrm{~mL} \times 3)$. The organic layer was combined and dried over anhydrous $\mathrm{MgSO}_{4}$, filtered, and concentrated under reduced pressure. The resulting mixture was purified by column chromatography on silica gel (petroleum ether: $\left.\mathrm{CH}_{2} \mathrm{Cl}_{2}=8: 1\right)$ to afford compound $\mathbf{k}(200 \mathrm{mg})$ in $69 \%$ yield as a pale green oil. ${ }^{1} \mathrm{H} \mathrm{NMR}\left(400 \mathrm{MHz}, \mathrm{CDCl}_{3}\right) \delta 7.56(\mathrm{~d}, J=9.6 \mathrm{~Hz}, 1 \mathrm{H}), 7.51(\mathrm{~d}, J=9.2 \mathrm{~Hz}$, $1 \mathrm{H}), 7.37(\mathrm{~d}, J=7.6 \mathrm{~Hz}, 1 \mathrm{H}), 7.28(\mathrm{~d}, J=15.9 \mathrm{~Hz}, 1 \mathrm{H}), 7.25-7.18(\mathrm{~m}, 2 \mathrm{H}), 7.04(\mathrm{~d}, J$ $=8 \mathrm{~Hz}, 1 \mathrm{H}), 7.00(\mathrm{~d}, J=16 \mathrm{~Hz}, 1 \mathrm{H}), 6.74(\mathrm{t}, J=7.7 \mathrm{~Hz}, 1 \mathrm{H}), 6.62(\mathrm{~d}, J=8.1 \mathrm{~Hz}, 1 \mathrm{H})$, $3.85(\mathrm{~s}, 1 \mathrm{H}), 2.80(\mathrm{~s}, 3 \mathrm{H}) .{ }^{13} \mathrm{C} \mathrm{NMR}\left(101 \mathrm{MHz}, \mathrm{CDCl}_{3}\right) \delta 145.6,136.4,131.9,128.3$, 128.1, 127.6, 126.5, 126.5, 126.3, 125.7, 122.9, 122.4, 116.3, 109.3, 29.7. HRMS (ESITOF) m/z: $[\mathrm{M}+\mathrm{H}]^{+}$calculated for $\mathrm{C}_{15} \mathrm{H}_{15} \mathrm{BrN} 288.0388$, found 288.0372.

\subsection{Synthesis of 4}<smiles>CNc1ccccc1/C=C/c1ccccc1Br</smiles>

$$
\text { i) } t \text {-BuLi, THF, }-78^{\circ} \mathrm{C}
$$<smiles>CNc1ccccc1/C=C/c1ccccc1S(C)(=O)=O</smiles>

4

A solution of $t$-BuLi in hexane $(1 \mathrm{~mL}, 1.3 \mathrm{M}, 1.3 \mathrm{mmol})$ was added drop wise by syringe at $-78{ }^{\circ} \mathrm{C}$ to a solution of $\mathbf{k}(0.151 \mathrm{~g}, 0.5 \mathrm{mmol})$ in anhydrous THF $(10 \mathrm{~mL})$. The mixture was stirred at the same temperature for $2 \mathrm{~h}$. A solution of dimesitylboron fluoride $(161 \mathrm{mg}, 0.6 \mathrm{mmol})$ in anhydrous THF $(5 \mathrm{~mL})$ was added to the reaction 
mixture using a syringe. The reaction mixture was warmed up to room temperature and stirred overnight. The reaction was quenched with a saturated brine and the aqueous layer was extracted with $\mathrm{CH}_{2} \mathrm{Cl}_{2}(20 \mathrm{~mL} \times 3)$. The organic layer was combined and dried over anhydrous $\mathrm{Na}_{2} \mathrm{SO}_{4}$, filtered, and concentrated under reduced pressure. The resulting mixture was purified by column chromatography on silica gel (petroleum ether: $\left.\mathrm{CH}_{2} \mathrm{Cl}_{2}=8: 1\right)$ to afford compound $4(800 \mathrm{mg})$ in $35 \%$ yield as a yellow green solid. m. p. $136-140{ }^{\circ} \mathrm{C} .{ }^{1} \mathrm{H}$ NMR $\left(400 \mathrm{MHz}, \mathrm{C}_{6} \mathrm{D}_{6}\right) \delta 7.57(\mathrm{~d}, J=7.9 \mathrm{~Hz}, 1 \mathrm{H}), 7.48(\mathrm{~d}$, $J=7.5 \mathrm{~Hz}, 1 \mathrm{H}), 7.40(\mathrm{~d}, J=16 \mathrm{~Hz}, \mathrm{HC}=\mathrm{CH}, 1 \mathrm{H}), 7.23(\mathrm{t}, J=8.1 \mathrm{~Hz}, 1 \mathrm{H}), 7.13(\mathrm{~d}, J=$ $8.8 \mathrm{~Hz}, 1 \mathrm{H}), 7.05(\mathrm{t}, J=7.9 \mathrm{~Hz}, 1 \mathrm{H}), 6.97(\mathrm{~d}, J=16 \mathrm{~Hz}, \mathrm{HC}=\mathrm{CH}, 1 \mathrm{H}), 6.79-6.69(\mathrm{~m}$, $6 \mathrm{H}), 6.46(\mathrm{~d}, J=8.0 \mathrm{~Hz}, 1 \mathrm{H}), 3.24(\mathrm{~s}, 1 \mathrm{H}), 2.35(\mathrm{~s}, 3 \mathrm{H}), 2.16(\mathrm{~s}, 6 \mathrm{H}), 2.13(\mathrm{~s}, 12 \mathrm{H}) .{ }^{13} \mathrm{C}$ NMR (101 MHz, $\left.\mathrm{C}_{6} \mathrm{D}_{6}\right) \delta 146.6,143.0,140.4,139.0,135.3,131.1,131.0,128.8,128.7$, $127.1,125.4,124.7,124.1,117.3,110.1,30.2,23.1,20.9 .{ }^{11} \mathrm{~B} \mathrm{NMR}\left(225 \mathrm{MHz}, \mathrm{C}_{6} \mathrm{D}_{6}\right)$ $\delta 76$ ppm. HRMS MALDI-MS (pos.) m/z: $\left[\mathrm{M}^{+}\right]$calculated for $\mathrm{C}_{33} \mathrm{H}_{36} \mathrm{BN}$ 457.2941, found 457.2931.

\section{Reference}

[1] Yong-gang Shi, Soren K. Mellerup, Kang Yuan, Guo-Fei Hu, Francoise Sauriol, Tai Peng, Nan Wang, Pangkuan Chen and Suning Wang, Chem. Sci. 2018, 9, 3844-3855.

[2] Fan Yang, Md. Akhtaruzzaman, Ashraful Islamc, Tienan Jin, Ahmed El-Shafei, Chuanjiang Qin, Liyuan Han, Khalid A. Alamry, Samia A. Kosa, Mahmoud A. Hussein, Abdullah Mohamed Asirie and Yoshinori Yamamoto, J. Mater. Chem. 2012, 22, 22550 22557.

[3] Ting-Jun Wang, Wen-Wen Chen, Yi Lia and Ming-Hua Xu, Org. Biomol. Chem. 2015, 13, 6580-6586.

[4] Takeru Torigoe, Toshimichi Ohmura, and Michinori Suginome, Angew. Chem. Int. Ed. 2017, 56, 14272-14276.

[5] Yi-Qiao Yan, Yan-Bang Li, Jian-Wu Wang, and Cui-Hua Zhao, Chem. Asian J. 2013, 12, 3164-3176.

[6] Sebastian Würtz, Claudia Lohre, Roland Fröhlich, Klaus Bergander, and Frank Glorius, J. Am. Chem. Soc. 2009, 131, 8344-8345. 


\section{Characterization by NMR and HRMS Spectroscopy}

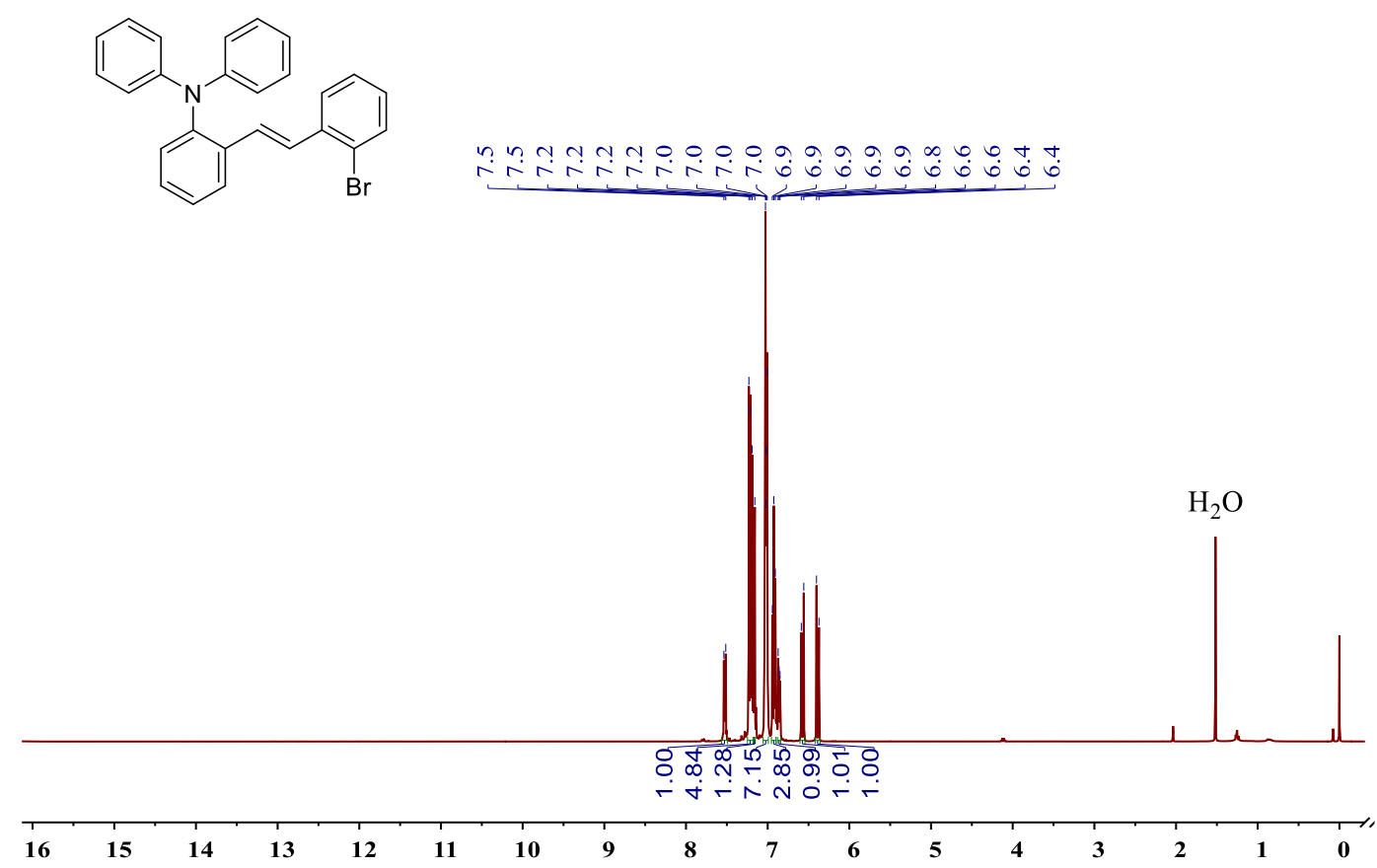

Figure S1. ${ }^{1} \mathrm{H} \mathrm{NMR}\left(400 \mathrm{MHz}, \mathrm{CDCl}_{3}\right)$ spectrum of c.

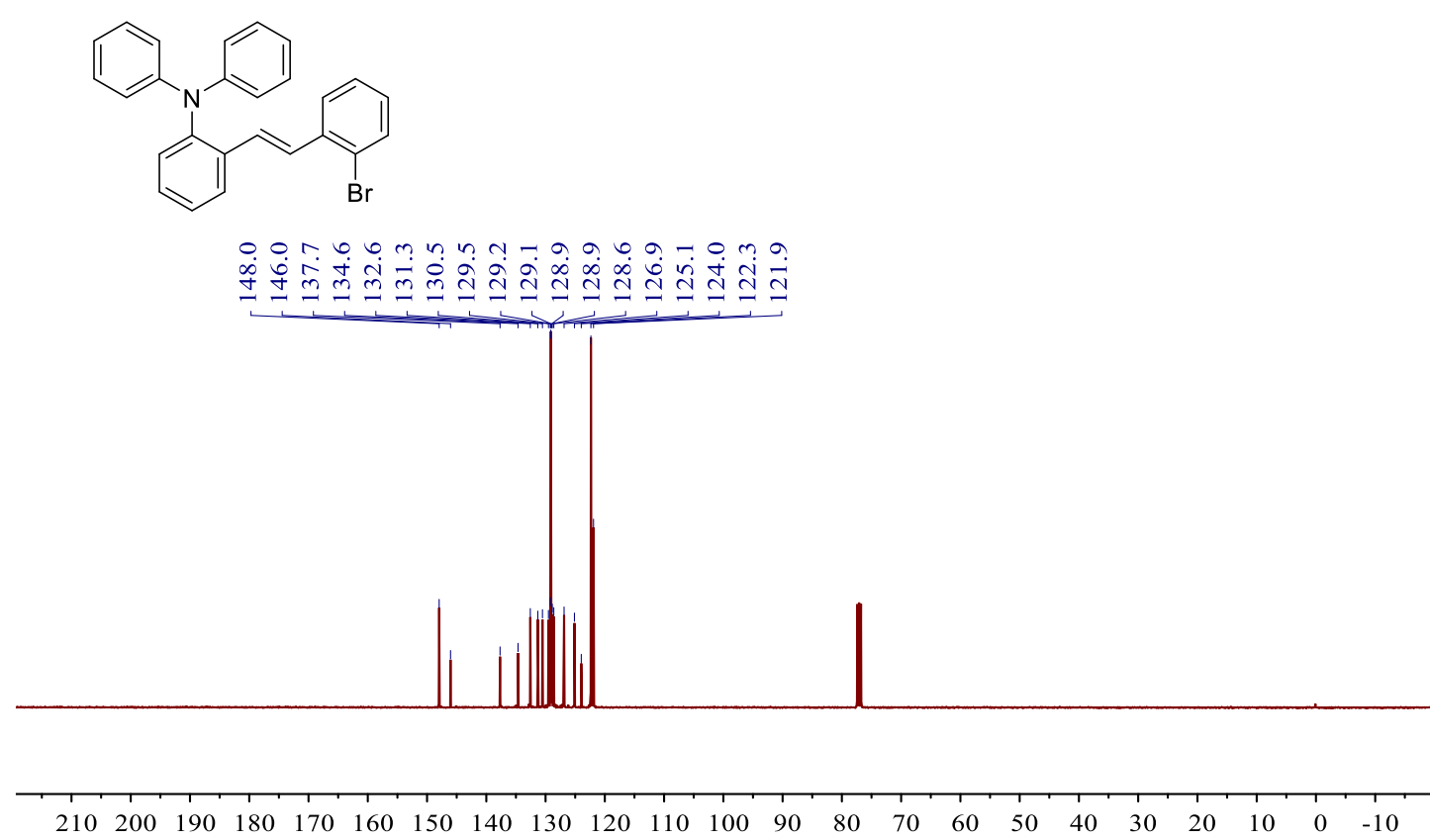

Figure S2. ${ }^{13} \mathrm{C}$ NMR $\left(101 \mathrm{MHz}, \mathrm{CDCl}_{3}\right)$ spectrum of c. 

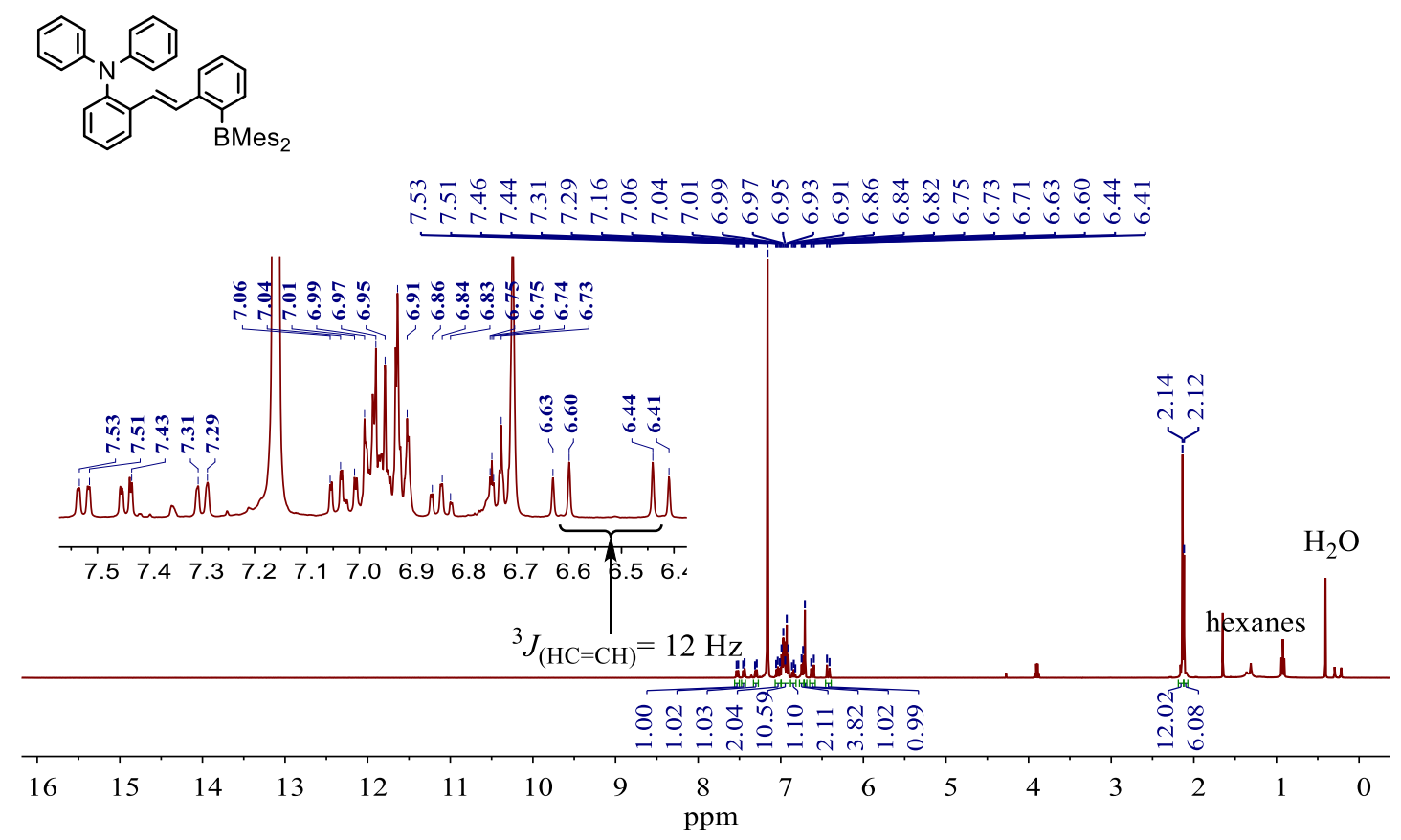

Figure S3. ${ }^{1} \mathrm{H}$ NMR $\left(400 \mathrm{MHz}, \mathrm{C}_{6} \mathrm{D}_{6}\right)$ spectrum of $\mathbf{1}$.

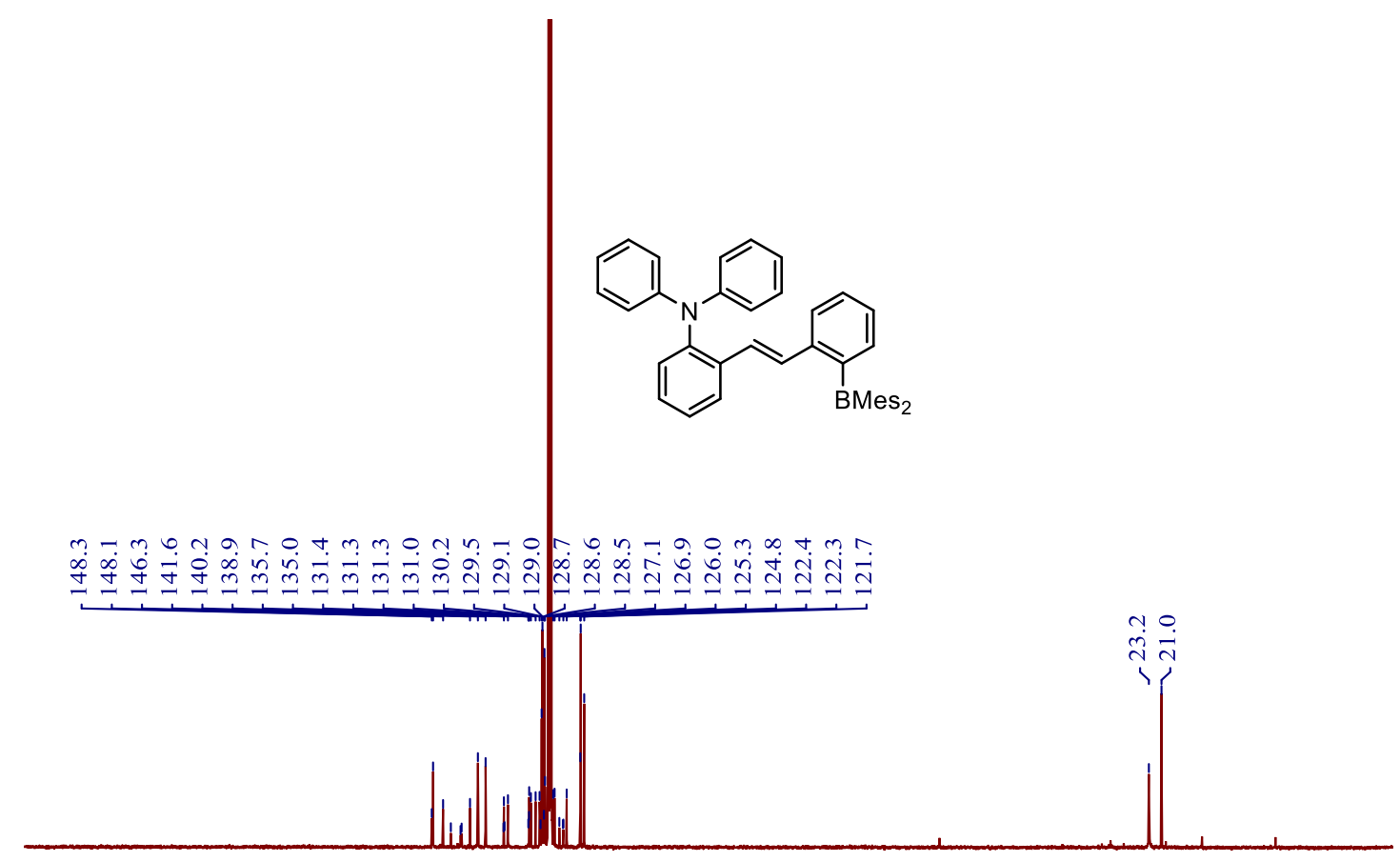

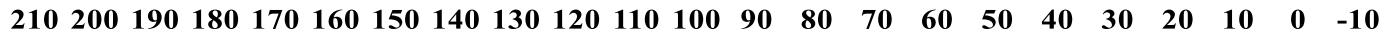

Figure S4. ${ }^{13} \mathrm{C}$ NMR (101 MHz, $\left.\mathrm{C}_{6} \mathrm{D}_{6}\right)$ spectrum of 1 . 


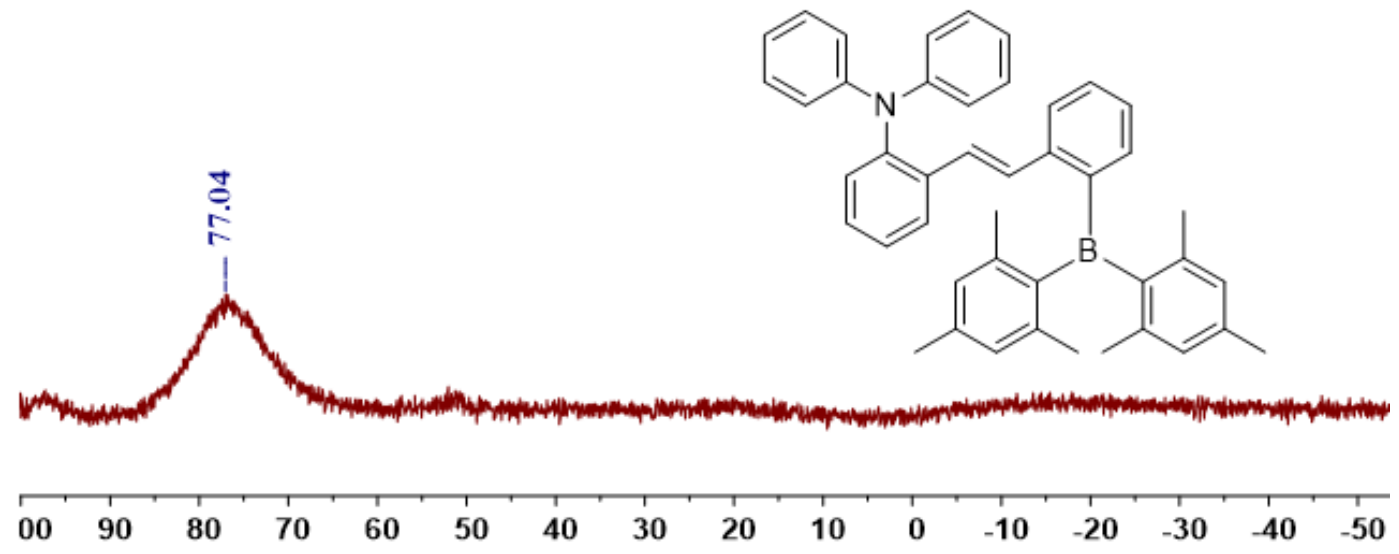

Figure S5. ${ }^{11} \mathrm{~B}$ NMR (225 MHz, $\left.\mathrm{C}_{6} \mathrm{D}_{6}\right)$ spectrum of $\mathbf{1}$.

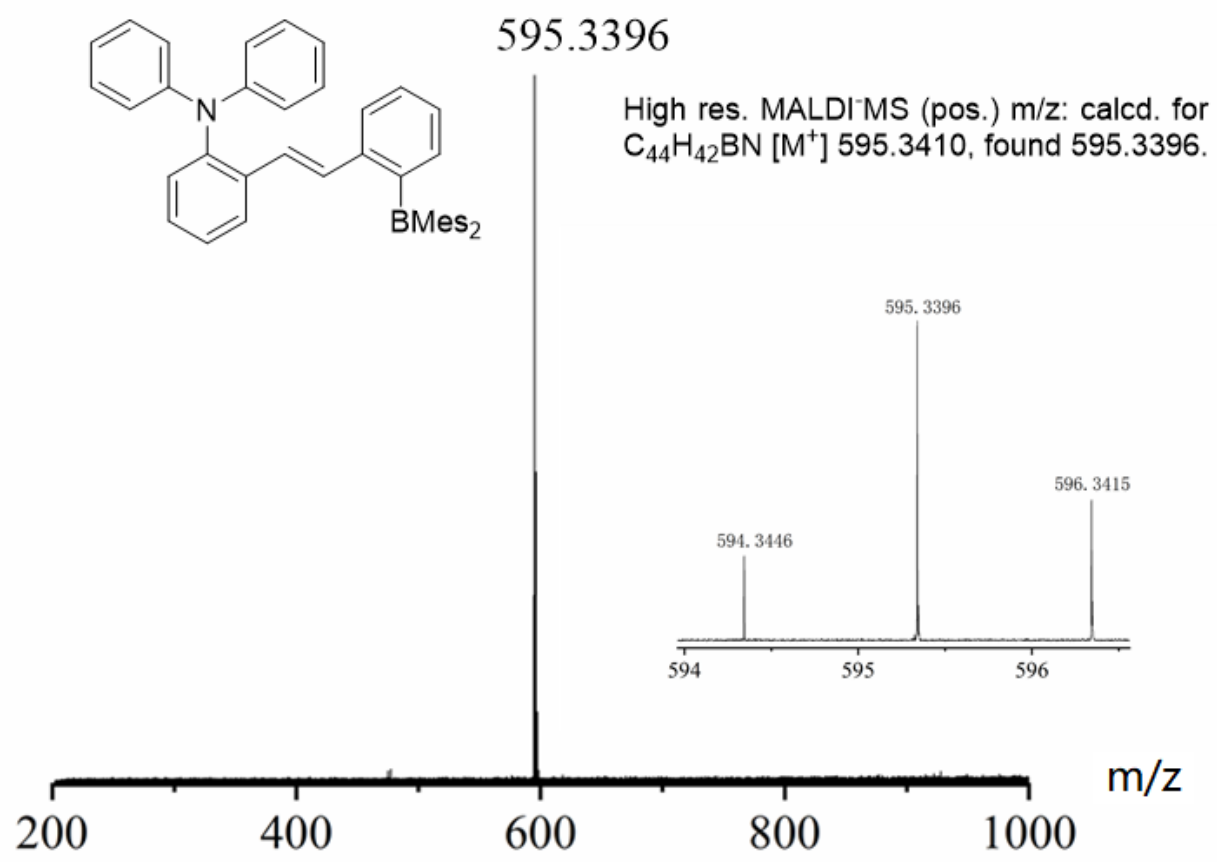

Figure S6. High-resolution MALDI-MS (positive) of 1. 


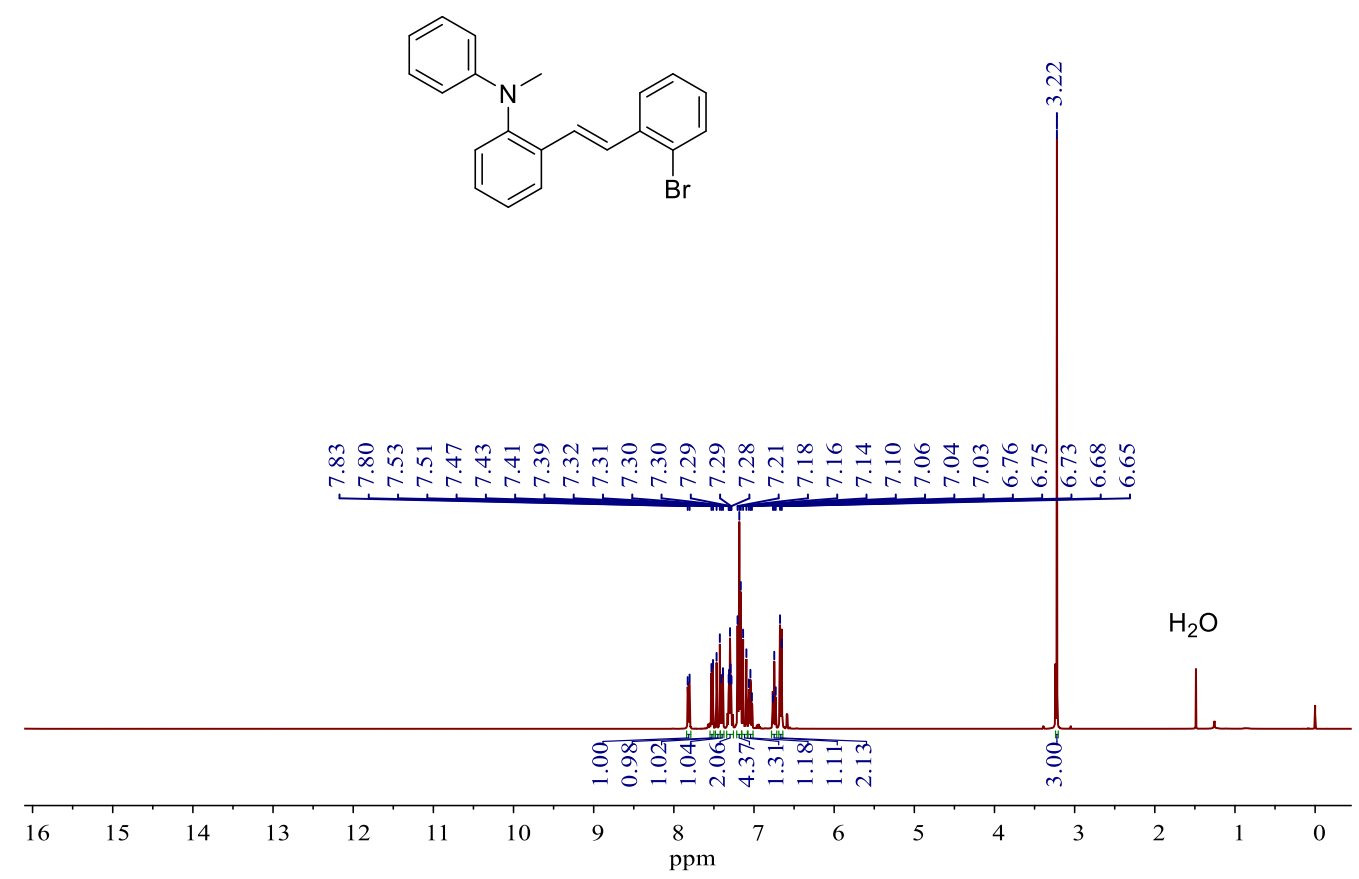

Figure S7. ${ }^{1} \mathrm{H} \mathrm{NMR}\left(400 \mathrm{MHz}, \mathrm{CDCl}_{3}\right)$ spectrum of $\mathbf{f}$.

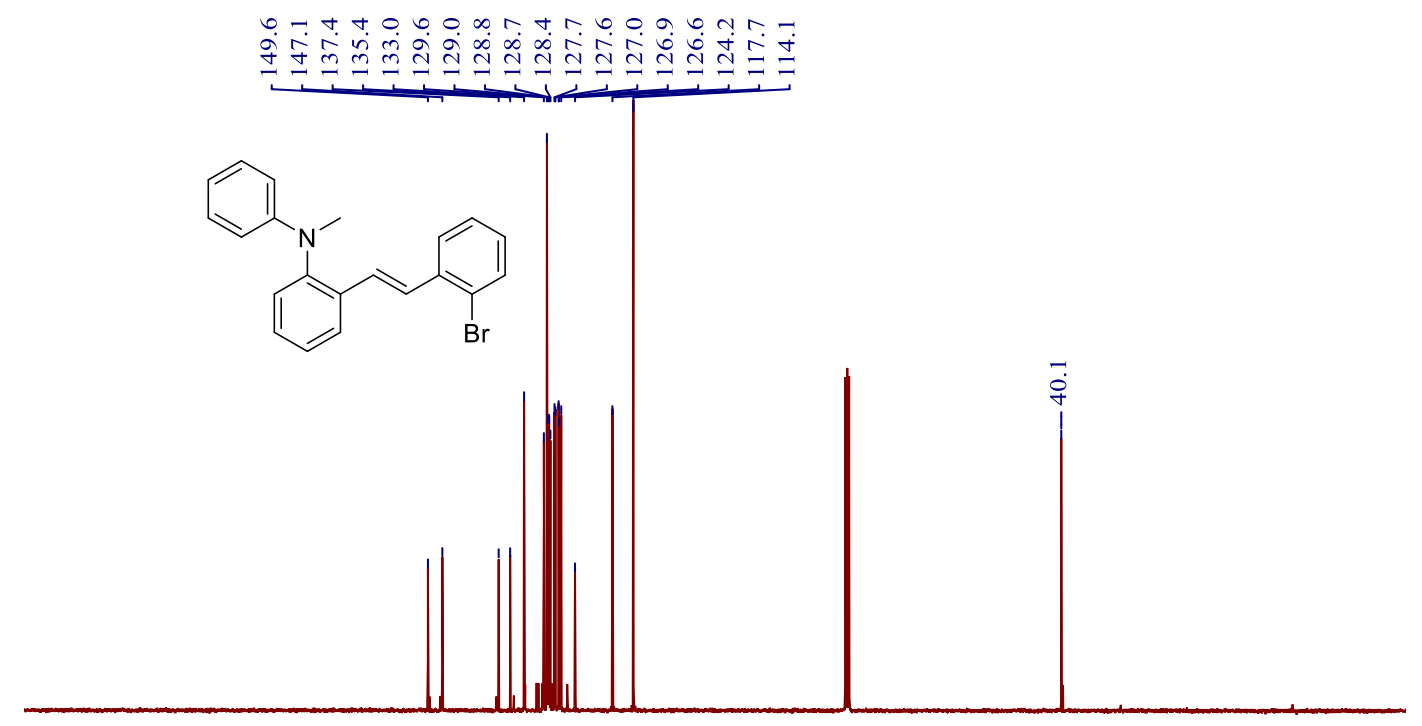

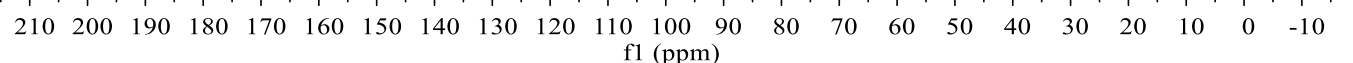

Figure S8. ${ }^{13} \mathrm{C}$ NMR $\left(101 \mathrm{MHz}, \mathrm{CDCl}_{3}\right)$ spectrum of $\mathbf{f}$. 


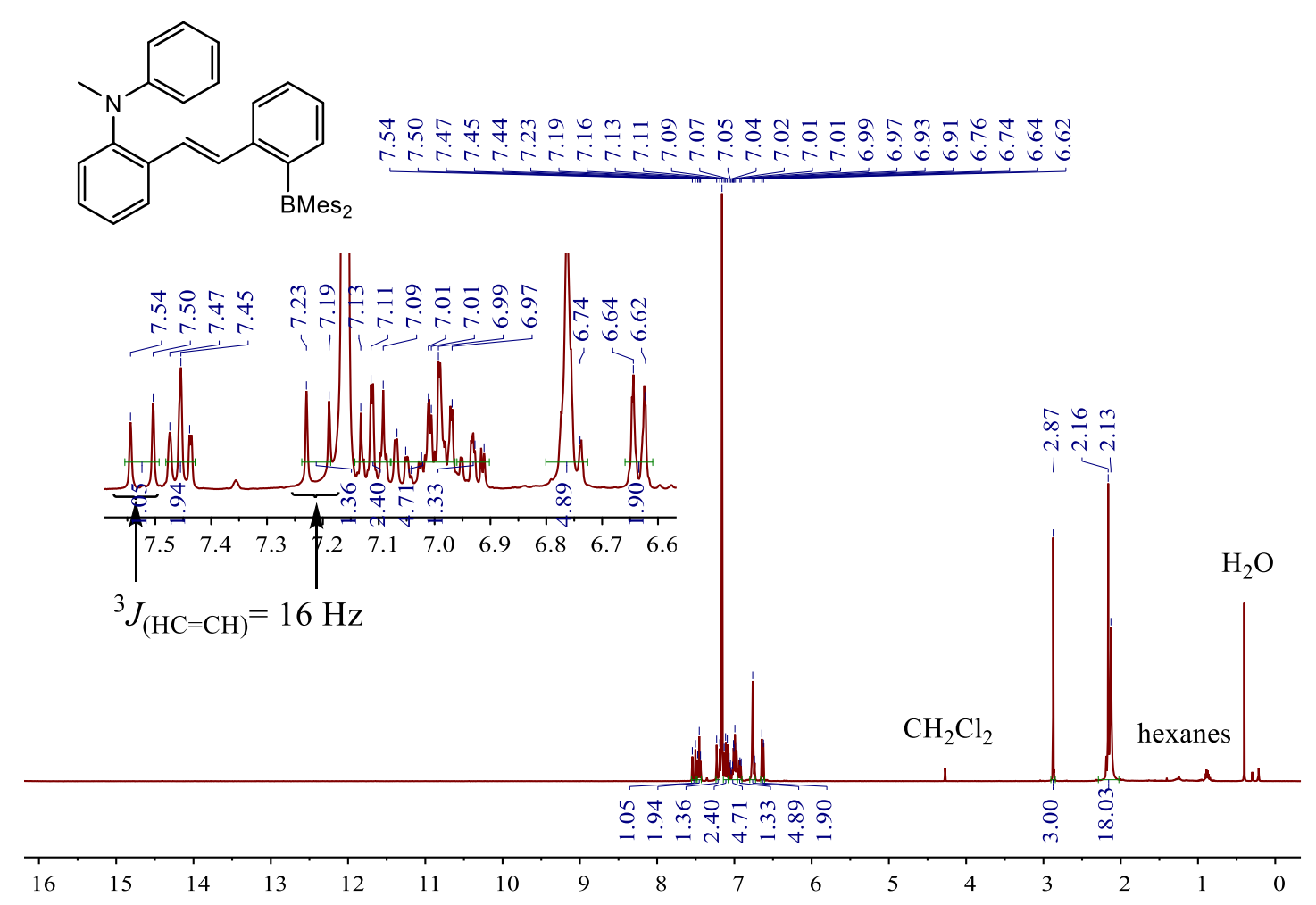

Figure S9. ${ }^{1} \mathrm{H}$ NMR (400 MHz, C6 6 ) spectrum of 2.

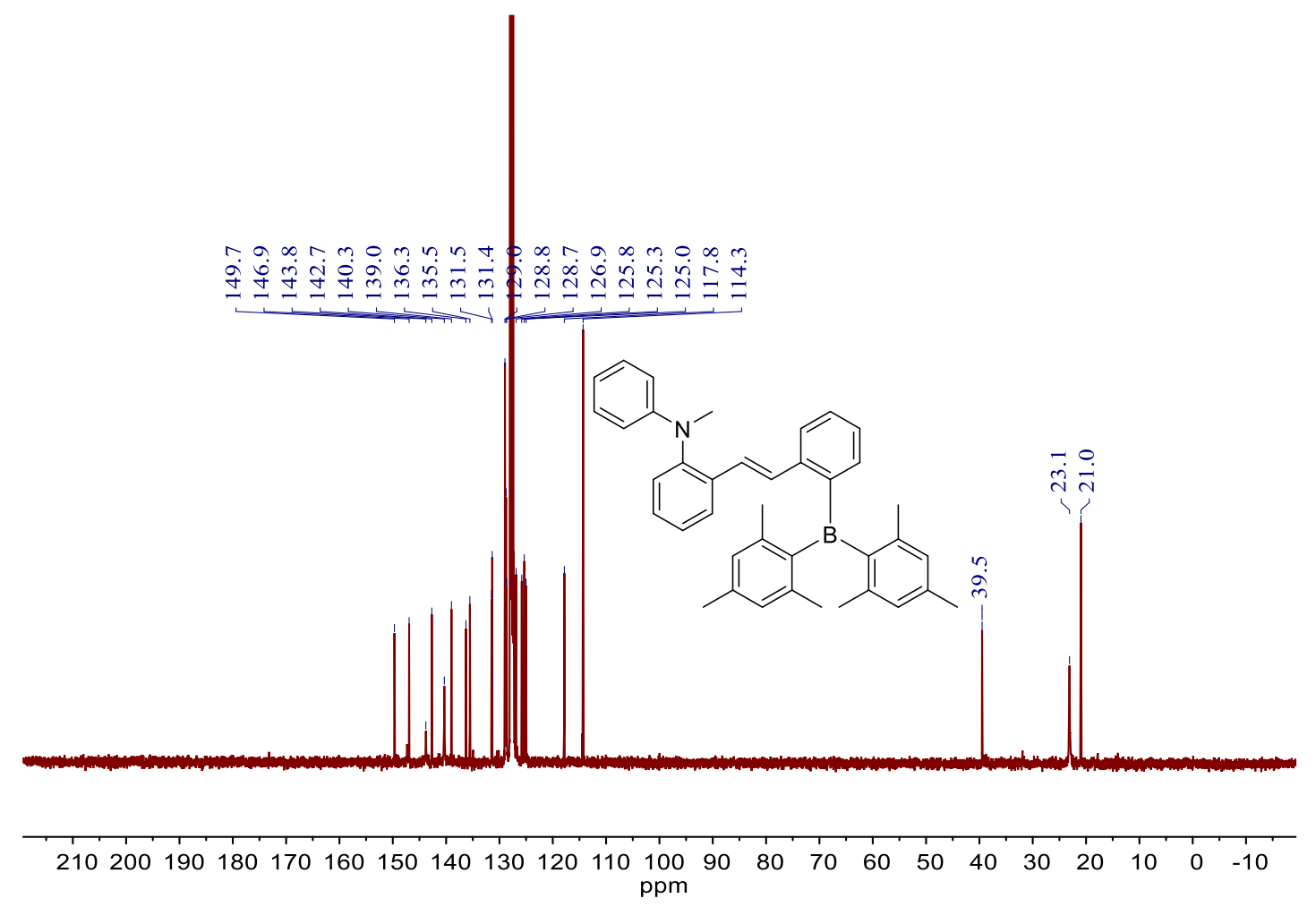

Figure S10. ${ }^{13} \mathrm{C}$ NMR (101 MHz, C6 6 ) spectrum of 2. 


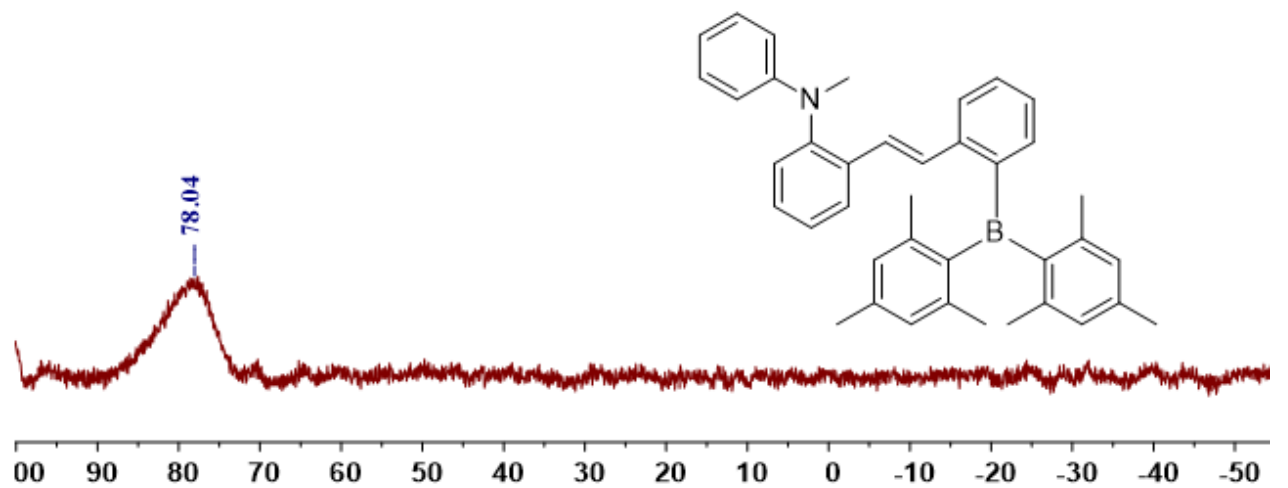

Figure S11. ${ }^{11} \mathrm{~B}$ NMR (225 MHz, $\left.\mathrm{C}_{6} \mathrm{D}_{6}\right)$ spectrum of 2.

533.3244

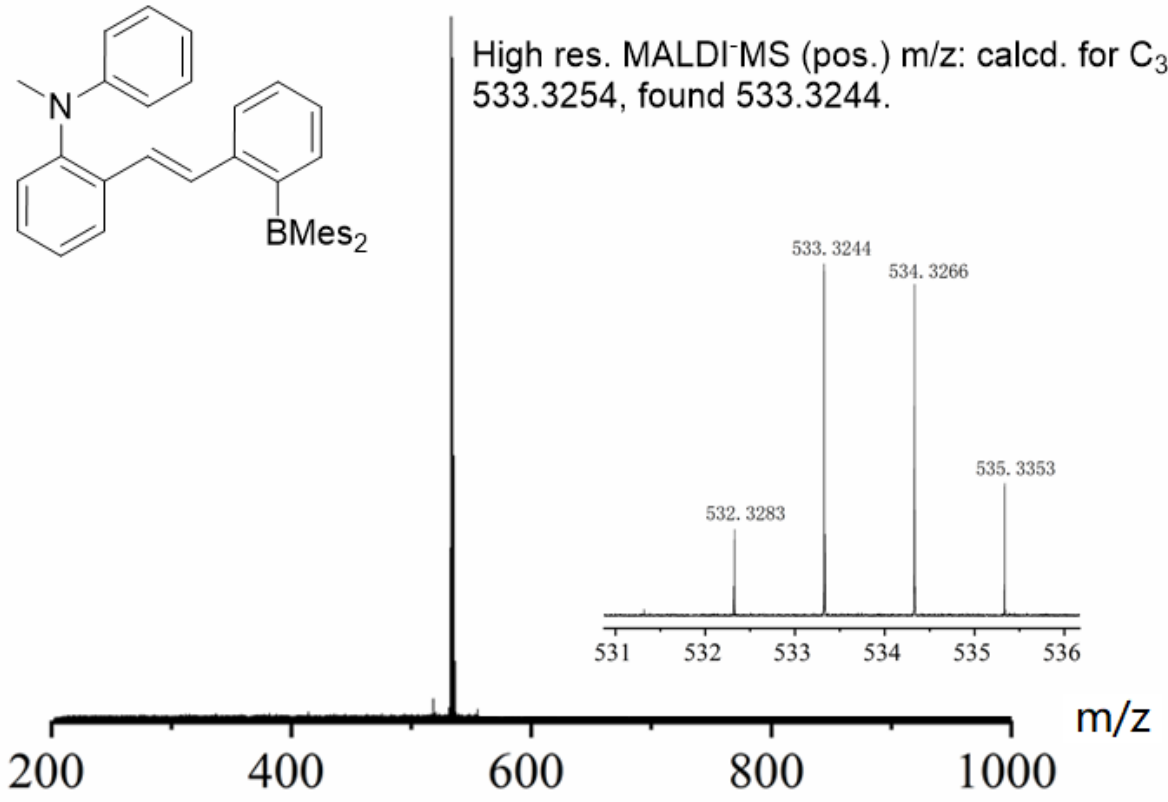

Figure S12. High-resolution MALDI-MS (positive) of 2.

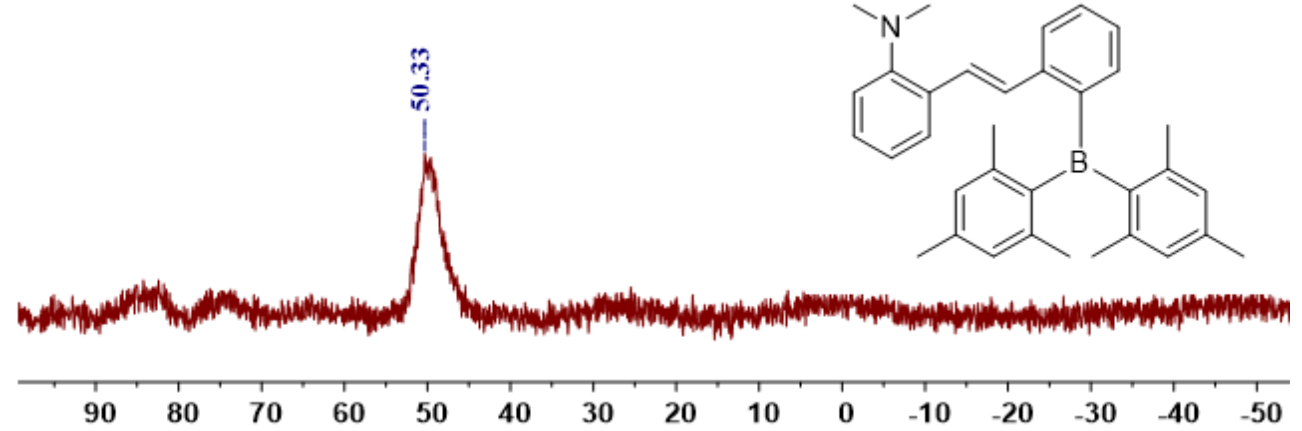

Figure S13. ${ }^{11} \mathrm{~B}$ NMR $\left(225 \mathrm{MHz}, \mathrm{C}_{6} \mathrm{D}_{6}\right)$ spectrum of 3. 


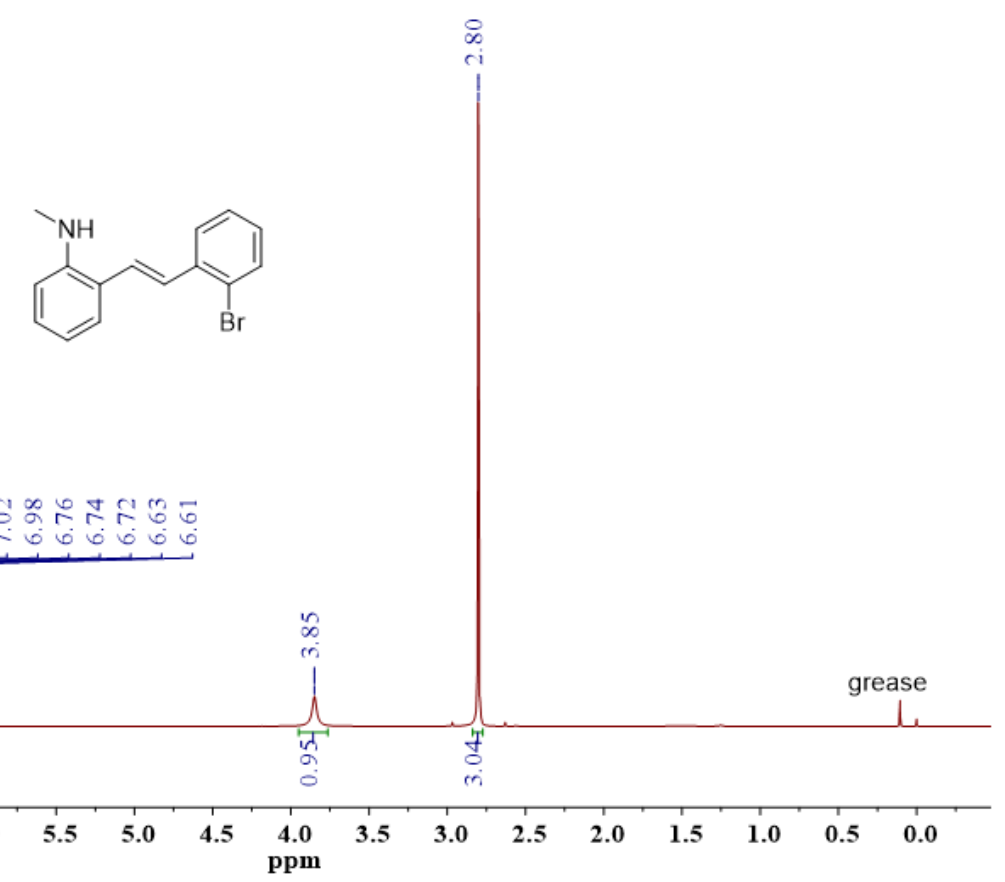

Figure S14. ${ }^{1} \mathrm{H} \mathrm{NMR}\left(400 \mathrm{MHz}, \mathrm{CDCl}_{3}\right)$ spectrum of $\mathbf{k}$.

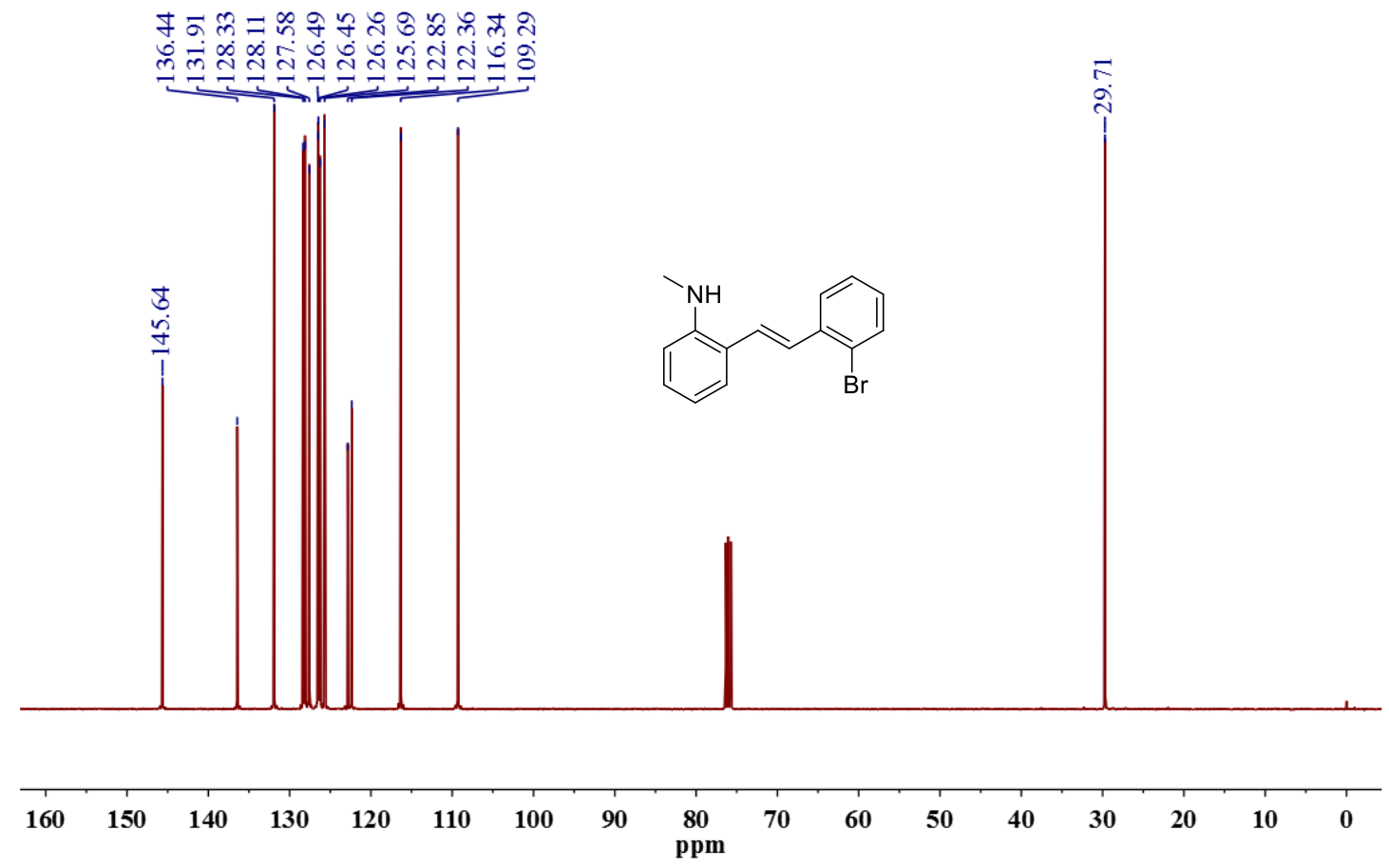

Figure S15. ${ }^{13} \mathrm{C}$ NMR (101 $\left.\mathrm{MHz}, \mathrm{CDCl}_{3}\right)$ spectrum of $\mathbf{k}$. 


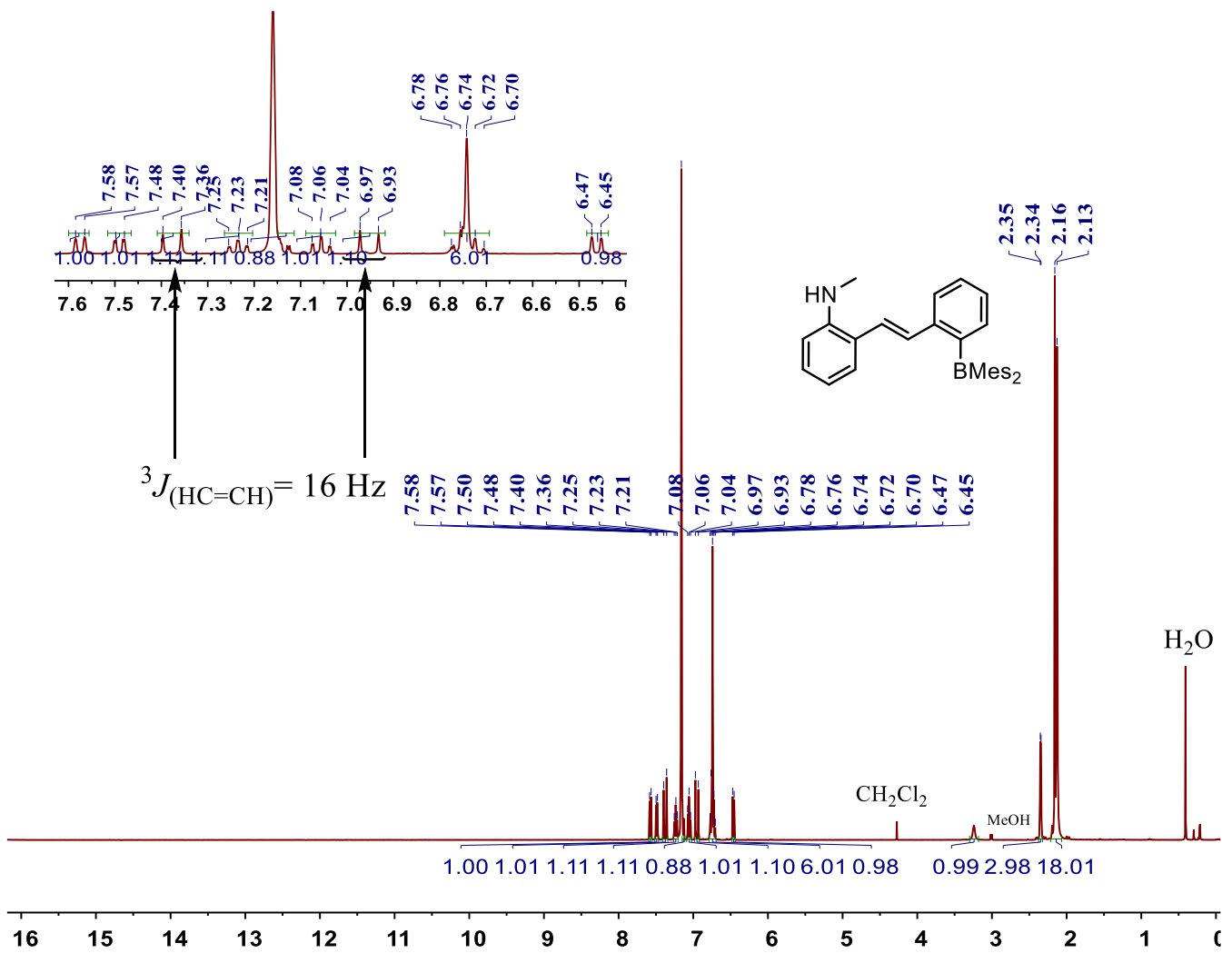

Figure S16. ${ }^{1} \mathrm{H}$ NMR (400 MHz, $\left.\mathrm{C}_{6} \mathrm{D}_{6}\right)$ spectrum of 4.

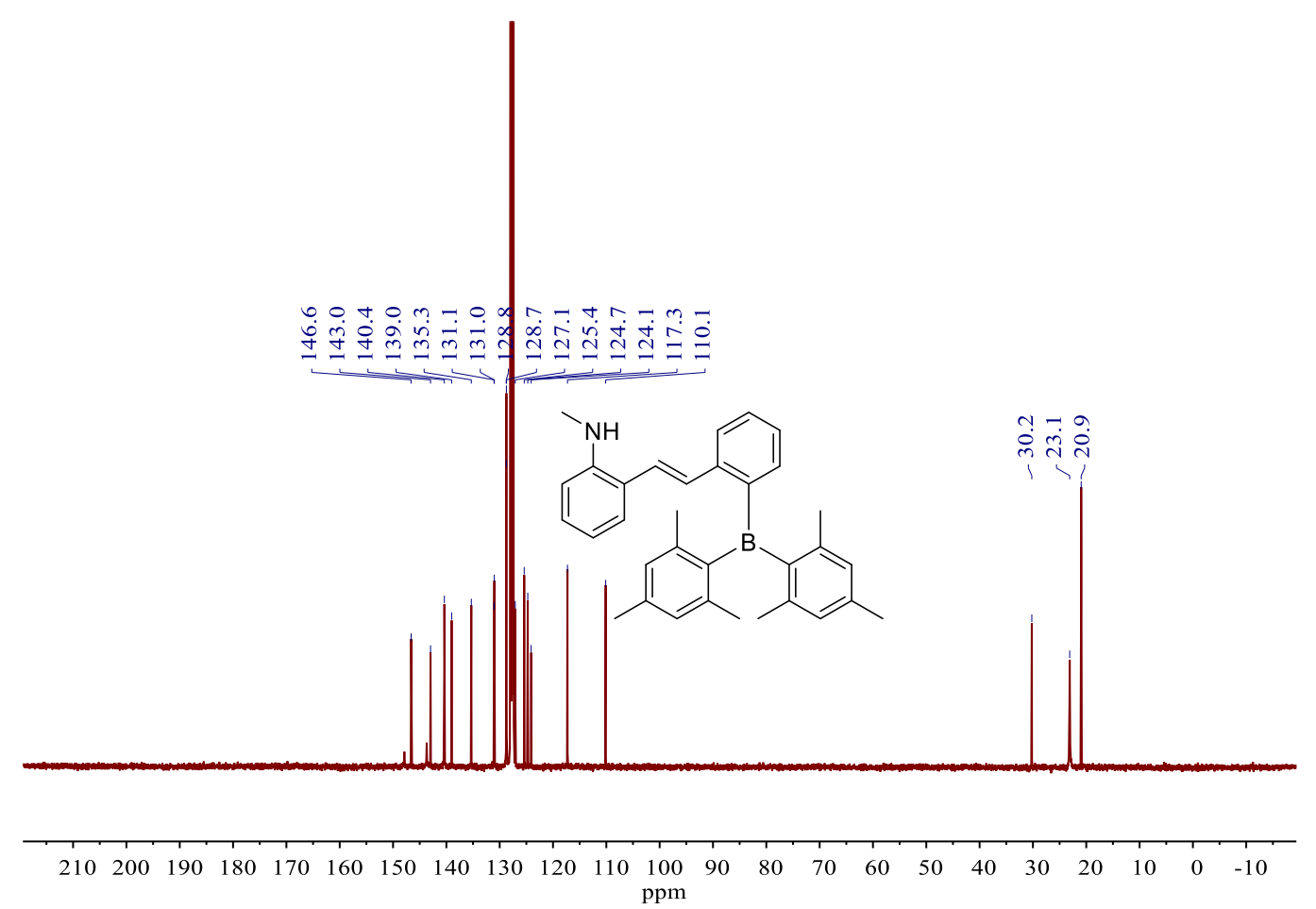

Figure S17. ${ }^{13} \mathrm{C}$ NMR (101 MHz, $\left.\mathrm{C}_{6} \mathrm{D}_{6}\right)$ spectrum of 4. 


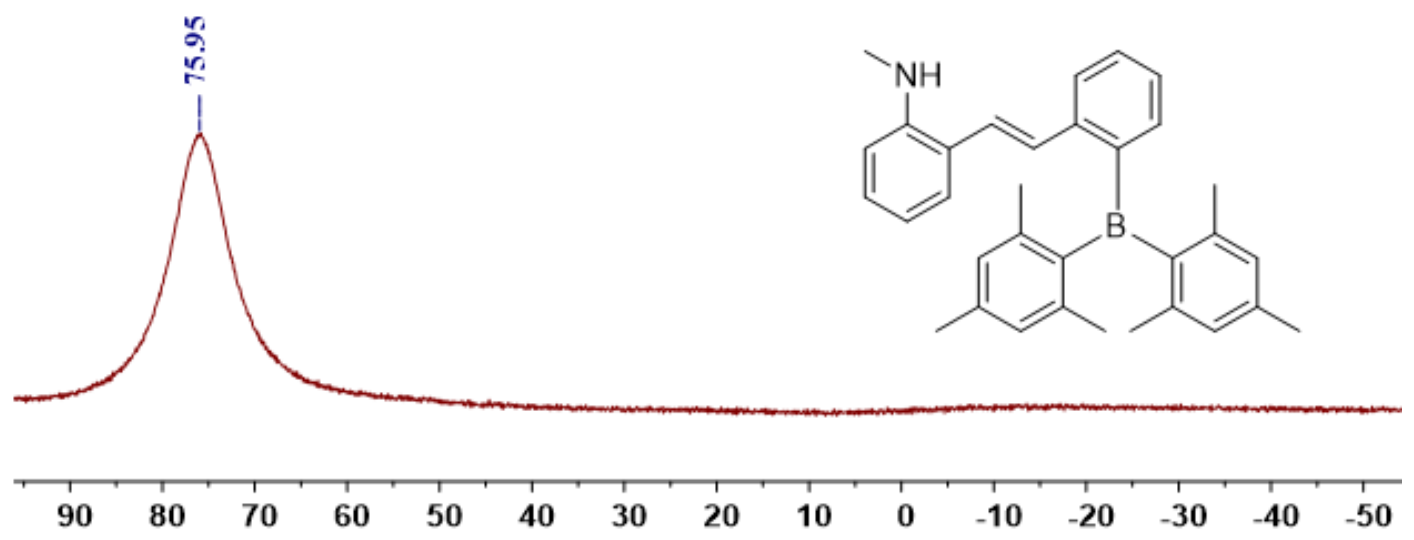

Figure S18. ${ }^{11} \mathrm{~B}$ NMR (225 MHz, $\left.\mathrm{C}_{6} \mathrm{D}_{6}\right)$ spectrum of 4 .

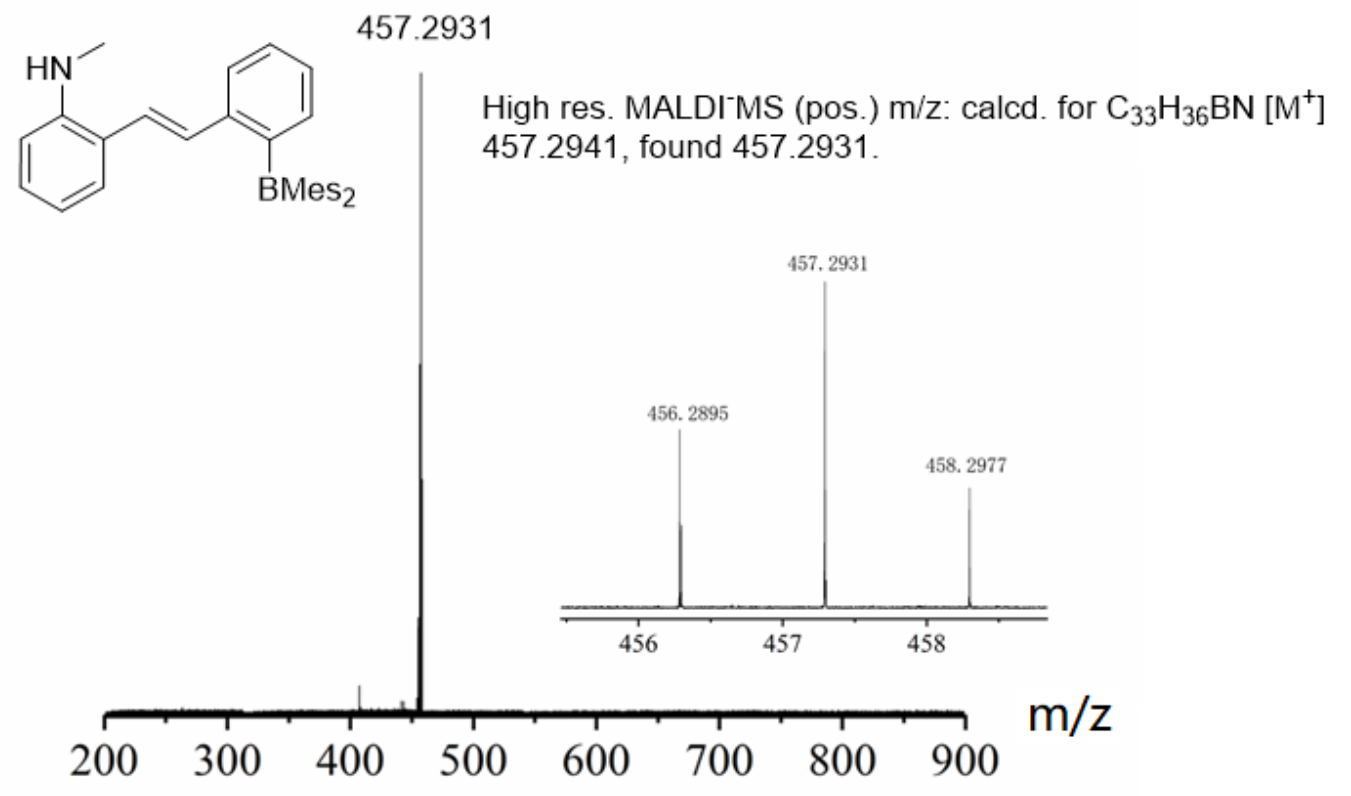

Figure S19. High-resolution MALDI-MS (positive) of 4. 


\section{XRD Measurements}

Table S1. Crystallographic data and structure refinement for 1, 2 and 4.

\begin{tabular}{|c|c|c|c|}
\hline Compound & 1 & 2 & 4 \\
\hline Empirical formula & $\mathrm{C}_{44} \mathrm{H}_{42} \mathrm{BN}_{8}$ & $\mathrm{C}_{39} \mathrm{H}_{40} \mathrm{BN}$ & $\mathrm{C}_{36} \mathrm{H}_{36} \mathrm{BN}$ \\
\hline Formula weight & 595.62 & 533.55 & 457.46 \\
\hline Temperature/K & 296.15 & 180 & 296 \\
\hline Crystal system & triclinic & monoclinic & triclinic \\
\hline Space group & P-1 & $\mathrm{P} 21_{1} / \mathrm{c}$ & P-1 \\
\hline Unit cell & $\begin{array}{l}\mathrm{a}=8.341(3) \AA \\
\mathrm{b}=8.604(3) \AA \\
\mathrm{c}=26.220(10) \AA \\
\alpha=92.451(11)^{\circ} \\
\beta=95.623(11)^{\circ} \\
\gamma=109.268(10)^{\circ}\end{array}$ & $\begin{array}{l}a=8.0082(4) \AA \\
b=23.6915(13) \AA \\
c=16.7958(10) \AA \\
\alpha=90^{\circ} \\
\beta=97.496(2)^{\circ} \\
\gamma=90^{\circ}\end{array}$ & $\begin{array}{l}a=9.2596(17) \AA \\
b=11.755(2) \AA \\
c=13.032(2) \AA \\
\alpha=79.423(6)^{\circ} \\
\beta=73.472(6)^{\circ} \\
\gamma=81.561(6)^{\circ}\end{array}$ \\
\hline Volume $/ \AA^{3}$ & $1762.1(11)$ & $3159.4(3)$ & $1330.0(4)$ \\
\hline $\mathrm{Z}$ & 2 & 4 & 2 \\
\hline$\rho_{\text {calc }} \mathrm{g} / \mathrm{cm}^{3}$ & 1.123 & 1.122 & 1.142 \\
\hline$\mu / \mathrm{mm}^{-1}$ & 0.063 & 0.063 & 0.064 \\
\hline $\mathrm{F}(000)$ & 636.0 & 1144.4 & 492.0 \\
\hline Crystal size $/ \mathrm{mm}^{3}$ & $0.2 \times 0.18 \times 0.18$ & $0.198 \times 0.143 \times 0.14$ & $0.3 \times 0.2 \times 0.1$ \\
\hline Radiation & $\operatorname{MoK} \alpha(\lambda=0.71073)$ & $\operatorname{MoK} \alpha(\lambda=0.71073)$ & $\operatorname{MoK} \alpha(\lambda=0.71073)$ \\
\hline 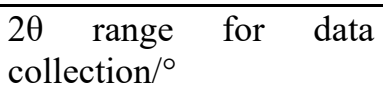 & $1.566-56.55$ & $4.22-55.28$ & $3.296-53.372$ \\
\hline Index ranges & $\begin{array}{l}-11 \leq \mathrm{h} \leq 11 \\
-11 \leq \mathrm{k} \leq 11 \\
-34 \leq 1 \leq 34\end{array}$ & $\begin{array}{c}-10 \leq \mathrm{h} \leq 10, \quad-29 \\
\leq \mathrm{k} \leq 30, \quad-21 \leq 1 \\
\leq 21\end{array}$ & $\begin{array}{c}-11 \leq \mathrm{h} \leq 11, \quad-14 \\
\leq \mathrm{k} \leq 14, \quad-16 \leq 1 \\
\leq 16\end{array}$ \\
\hline Reflections collected & 20902 & 42337 & 14095 \\
\hline Independent reflections & $\begin{array}{c}8652\left[R_{\text {int }}=0.0320,\right. \\
\left.R_{\text {sigma }}=0.0456\right]\end{array}$ & $\begin{array}{c}7322\left[R_{\text {int }}=0.0841,\right. \\
\left.R_{\text {sigma }}=0.0616\right]\end{array}$ & $\begin{array}{c}5470\left[R_{\text {int }}=0.0400,\right. \\
\left.R_{\text {sigma }}=0.0653\right]\end{array}$ \\
\hline $\begin{array}{c}\text { Data/restraints/paramete } \\
\text { rs }\end{array}$ & $8652 / 72 / 421$ & $7322 / 0 / 377$ & $5470 / 0 / 323$ \\
\hline Goodness-of-fit on $\mathrm{F}^{2}$ & 1.069 & 1.075 & 1.038 \\
\hline $\begin{array}{c}\text { Final R indexes }[\mathrm{I}>=2 \sigma \\
(\mathrm{I})]\end{array}$ & $\begin{array}{c}\mathrm{R}_{1}=0.0740, \\
\mathrm{wR}_{2}=0.1746\end{array}$ & $\begin{array}{l}R_{1}=0.0609 \\
\mathrm{wR}_{2}=0.1553\end{array}$ & $\begin{array}{l}\mathrm{R}_{1}=0.0552 \\
\mathrm{wR}_{2}=0.1367\end{array}$ \\
\hline $\begin{array}{c}\text { Final } \mathrm{R} \text { indexes [all } \\
\text { data] }\end{array}$ & $\begin{array}{c}\mathrm{R}_{1}=0.0940 \\
\mathrm{wR}_{2}=0.1848\end{array}$ & $\begin{array}{c}\mathrm{R}_{1}=0.1137 \\
\mathrm{wR}_{2}=0.1820\end{array}$ & $\begin{array}{c}\mathrm{R}_{1}=0.1024 \\
\mathrm{wR}_{2}=0.1569\end{array}$ \\
\hline $\begin{array}{l}\text { Largest diff. peak/hole } \\
\left(\text { e. } \AA^{-3}\right)\end{array}$ & $0.41 /-0.30$ & $0.40 /-0.38$ & $0.48 /-0.47$ \\
\hline
\end{tabular}

Table S2. Selected bond distances ( $⿱$ A) from X-ray data for 1, 2 and 4.

\begin{tabular}{|l|c|l|c|l|c|}
\hline \multicolumn{2}{|c|}{} & \multicolumn{2}{c|}{2} & \multicolumn{2}{c|}{4} \\
\hline Bond & Bond Length & Bond & Bond Length & Bond & Bond Length \\
\hline $\mathrm{N}(1)-\mathrm{C}(39)$ & $1.410(3)$ & $\mathrm{N}(1)-\mathrm{C}(7)$ & $1.406(2)$ & $\mathrm{N}(1)-\mathrm{C}(32)$ & $1.394(3)$ \\
\hline $\mathrm{N}(1)-\mathrm{C}(32)$ & $1.431(3)$ & $\mathrm{N}(1)-\mathrm{C}(9)$ & $1.415(2)$ & $\mathrm{N}(1)-\mathrm{C}(33)$ & $1.453(3)$ \\
\hline
\end{tabular}




\begin{tabular}{|c|l|l|l|l|l|}
\hline $\mathrm{N}(1)-\mathrm{C}(33)$ & $1.419(3)$ & $\mathrm{N}(1)-\mathrm{C}(8)$ & $1.448(2)$ & $\mathrm{C}(25)-\mathrm{C}(26)$ & $1.336(3)$ \\
\hline $\mathrm{C}(25)-\mathrm{C}(26)$ & $1.332(3)$ & $\mathrm{C}(15)-\mathrm{C}(16)$ & $1.326(2)$ & $\mathrm{C}(5)-\mathrm{B}(1)$ & $1.573(3)$ \\
\hline $\mathrm{C}(1)-\mathrm{B}(1)$ & $1.567(3)$ & $\mathrm{C}(32)-\mathrm{B}(1)$ & $1.575(3)$ & $\mathrm{C}(14)-\mathrm{B}(1)$ & $1.582(3)$ \\
\hline $\mathrm{C}(19)-\mathrm{B}(1)$ & $1.579(3)$ & $\mathrm{C}(23)-\mathrm{B}(1)$ & $1.582(3)$ & $\mathrm{C}(19)-\mathrm{B}(1)$ & $1.577(3)$ \\
\hline $\mathrm{C}(10)-\mathrm{B}(1)$ & $1.576(3)$ & $\mathrm{C}(22)-\mathrm{B}(1)$ & $1.567(3)$ & & \\
\hline
\end{tabular}

Table S3. Selected bond angles $\left(^{\circ}\right)$ from X-ray data for $\mathbf{1 ,} 2$ and 4.

\begin{tabular}{llll}
\hline $\mathbf{1}$ & Bond Angles & & Bond Angles \\
\hline $\mathrm{C}(39)-\mathrm{N}(1)-\mathrm{C}(32)$ & $118.51(17)$ & $\mathrm{C}(1)-\mathrm{B}(1)-\mathrm{C}(19)$ & $122.24(18)$ \\
$\mathrm{C}(39)-\mathrm{N}(1)-\mathrm{C}(33)$ & $121.98(18)$ & $\mathrm{C}(1)-\mathrm{B}(1)-\mathrm{C}(10)$ & $123.38(18)$ \\
$\mathrm{C}(33)-\mathrm{N}(1)-\mathrm{C}(32)$ & $118.62(17)$ & $\mathrm{C}(10)-\mathrm{B}(1)-\mathrm{C}(19)$ & $114.32(18)$ \\
$\mathrm{C}(26)-\mathrm{C}(25)-\mathrm{C}(24)$ & $128.0(2)$ & $\mathrm{C}(25)-\mathrm{C}(26)-\mathrm{C}(27)$ & $129.1(2)$ \\
\hline $\mathbf{2}$ & Bond Angles & & Bond Angles \\
\hline $\mathrm{C}(9)-\mathrm{N}(1)-\mathrm{C}(7)$ & $120.06(15)$ & $\mathrm{C}(23)-\mathrm{B}(1)-\mathrm{C}(32)$ & $120.32(16)$ \\
$\mathrm{C}(8)-\mathrm{N}(1)-\mathrm{C}(7)$ & $119.21(17)$ & $\mathrm{C}(22)-\mathrm{B}(1)-\mathrm{C}(32)$ & $122.85(16)$ \\
$\mathrm{C}(8)-\mathrm{N}(1)-\mathrm{C}(9)$ & $118.31(16)$ & $\mathrm{C}(22)-\mathrm{B}(1)-\mathrm{C}(23)$ & $116.75(15)$ \\
$\mathrm{C}(14)-\mathrm{C}(15)-\mathrm{C}(16)$ & $126.35(17)$ & $\mathrm{C}(15)-\mathrm{C}(16)-\mathrm{C}(17)$ & $126.05(17)$ \\
\hline $\mathbf{4}$ & Bond Angles & & Bond Angles \\
\hline $\mathrm{C}(32)-\mathrm{N}(1)-\mathrm{C}(33)$ & $120.2(2)$ & $\mathrm{C}(26)-\mathrm{C}(25)-\mathrm{C}(24)$ & $128.9(2)$ \\
$\mathrm{C}(25)-\mathrm{C}(26)-\mathrm{C}(27)$ & $124.8(2)$ & $\mathrm{C}(5)-\mathrm{B}(1)-\mathrm{C}(14)$ & $123.80(19)$ \\
$\mathrm{C}(5)-\mathrm{B}(1)-\mathrm{C}(19)$ & $117.51(18)$ & $\mathrm{C}(19)-\mathrm{B}(1)-\mathrm{C}(14)$ & $118.66(19)$
\end{tabular}

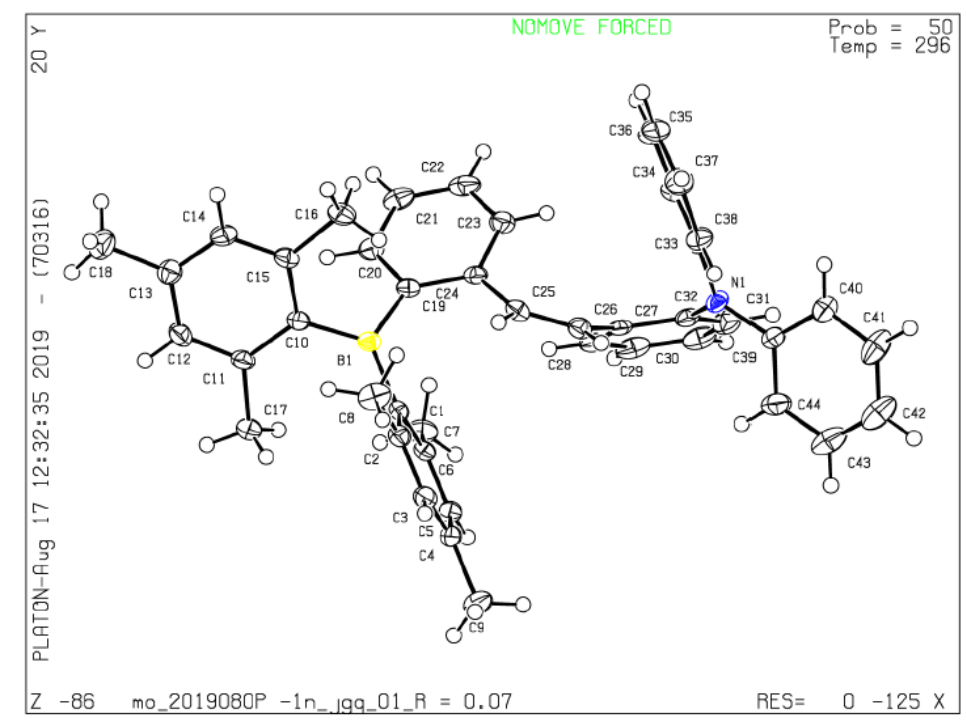

Figure S20. Thermal ellipsoid plot of $\mathbf{1}$ drawn at the contour probability of $50 \%$. 


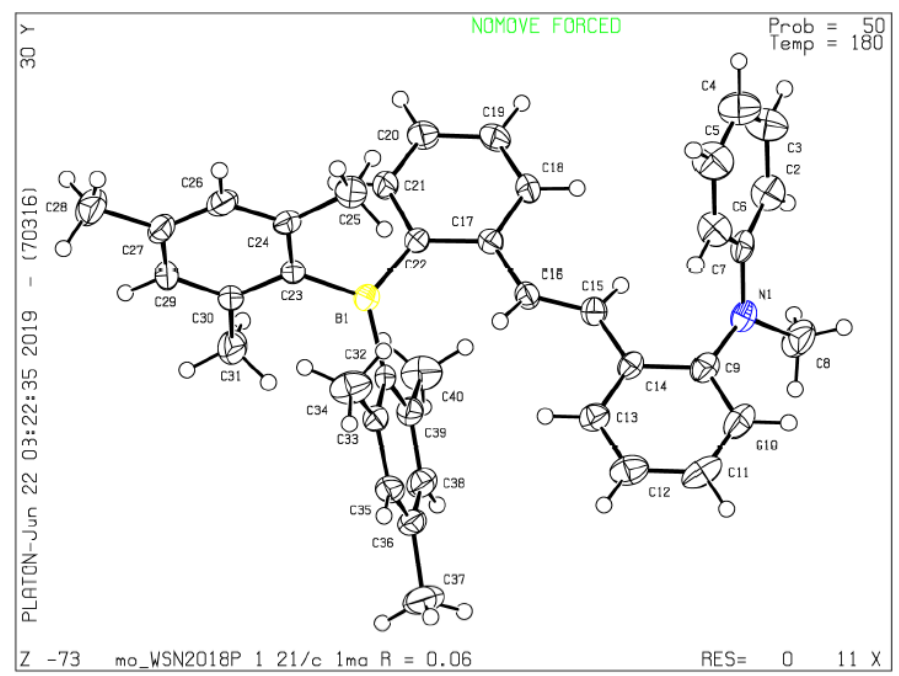

Figure S21. Thermal ellipsoid plot of $\mathbf{2}$ drawn at the contour probability of 50\%.

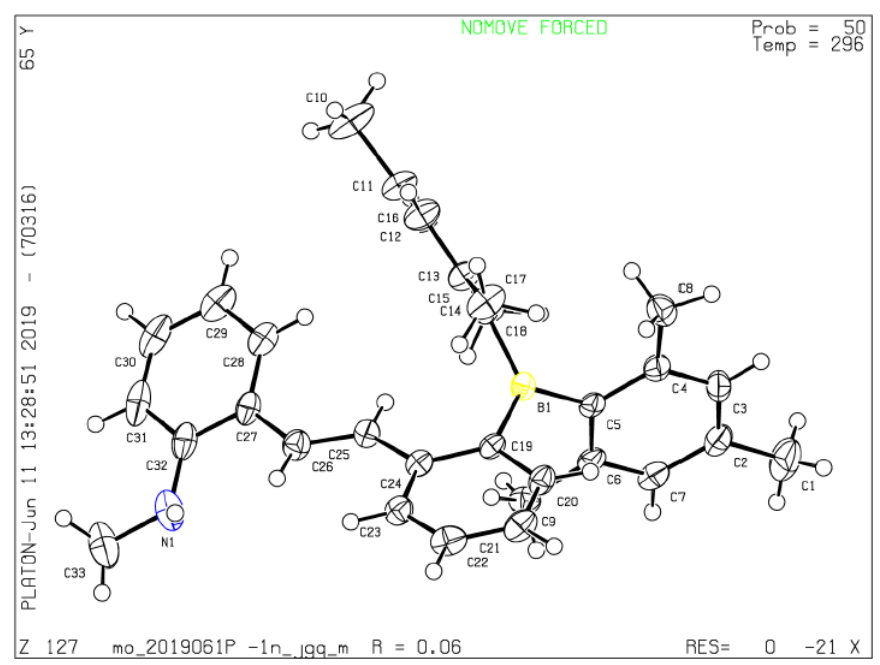

Figure S22. Thermal ellipsoid plot of 4 drawn at the contour probability of $50 \%$. 


\section{Electronic Structure Examination}

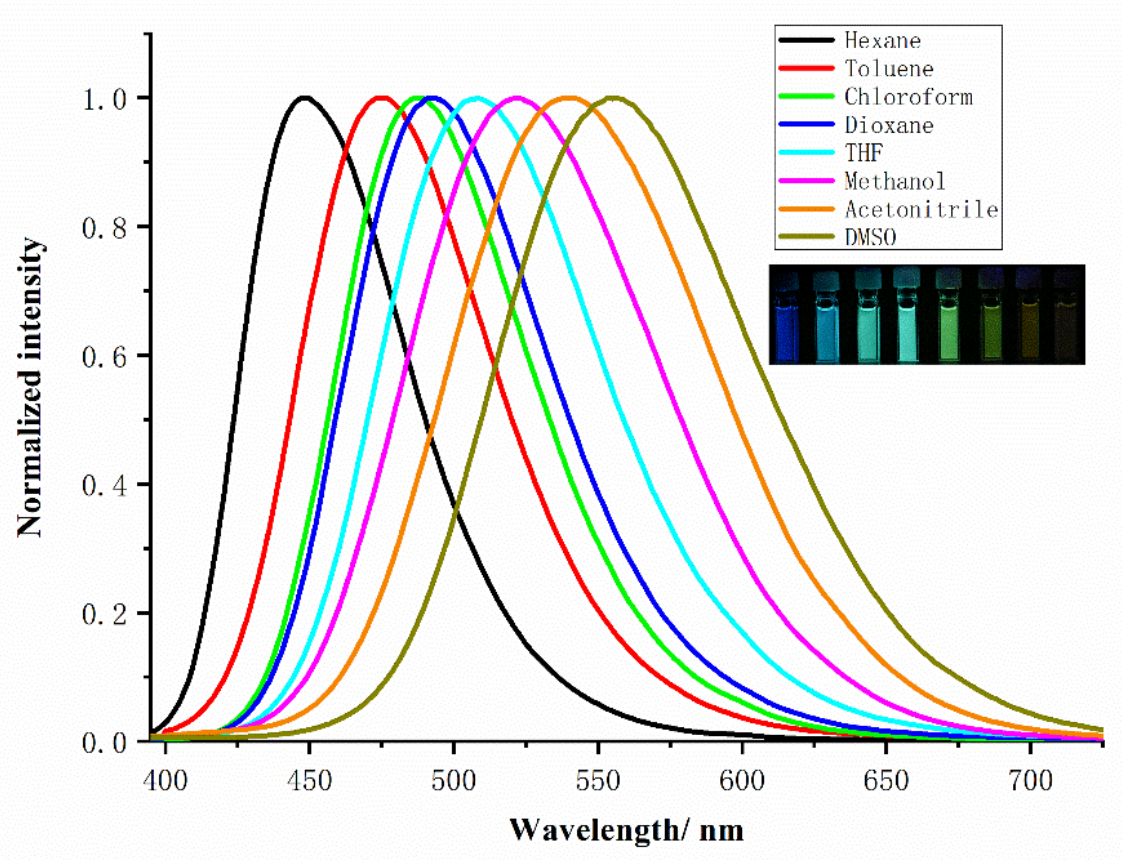

Figure S23. Solvatochromic emission spectra of $\mathbf{2}$ in different solvents of polarity $(c=$ $1.0 \times 10^{-5} \mathrm{M}, \lambda_{\mathrm{ex}}=300 \mathrm{~nm}$ ) at room temperture.

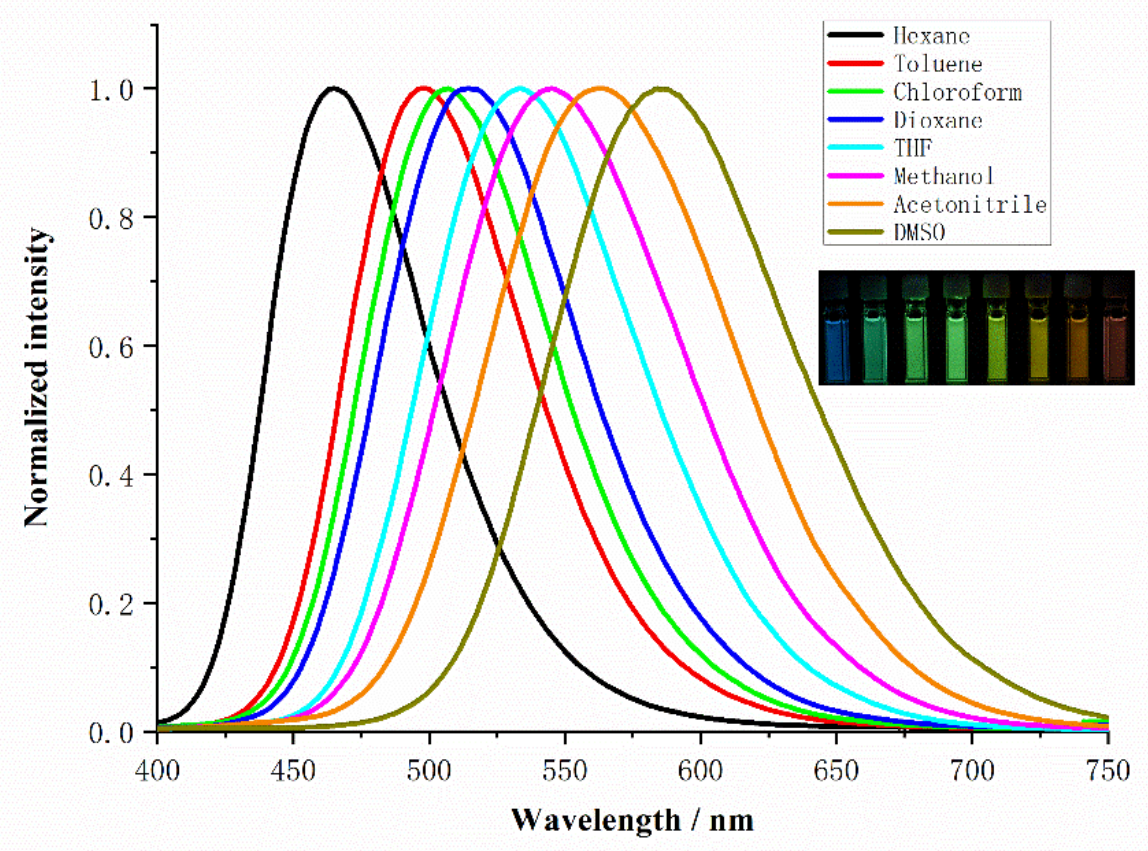

Figure S24. Solvatochromic emission spectra of $\mathbf{3}$ in different solvents of polarity $(c=$ $1.0 \times 10^{-5} \mathrm{M}, \lambda_{\mathrm{ex}}=311 \mathrm{~nm}$ ) at room temperture. 
Table S4. Emission (nm) of $\mathbf{1}-\mathbf{4}$ in different solvents $\left(c=1.0 \times 10^{-5} \mathrm{M}, \lambda_{\mathrm{ex}}=\lambda_{\mathrm{abs}(\max )}\right)$ and half liftetimes $\tau_{\mathrm{av}}(\mathrm{ns})$ in THF solutions.

\begin{tabular}{|c|c|c|c|c|}
\hline Solvent & $\mathbf{1}$ & $\mathbf{2}$ & $\mathbf{3}$ & $\mathbf{4}$ \\
\hline Hexane & 449 & 449 & 465 & 489 \\
\hline Toluene & 474 & 477 & 498 & 495 \\
\hline $\mathrm{CHCl}_{3}$ & 490 & 489 & 507 & 510 \\
\hline Dioxane & 487 & 492 & 515 & 515 \\
\hline $\mathrm{MeOH}$ & 509 & 521 & 545 & 550 \\
\hline $\mathrm{CH}_{3} \mathrm{CN}$ & 531 & 542 & 561 & 563 \\
\hline $\mathrm{DMSO}$ & 536 & 556 & 579 & 582 \\
\hline $\mathrm{THF} / \tau_{\mathrm{av}}(\mathrm{ns})$ & $498 / 4.2$ & $508 / 7.8$ & $533 / 7.3$ & $540 / 7.3$ \\
\hline $\mathrm{Film} / \Phi_{\mathrm{s}}(\%)$ & $472 / 57$ & $477 / 50$ & $492 / 82$ & $496 / 51$ \\
\hline
\end{tabular}
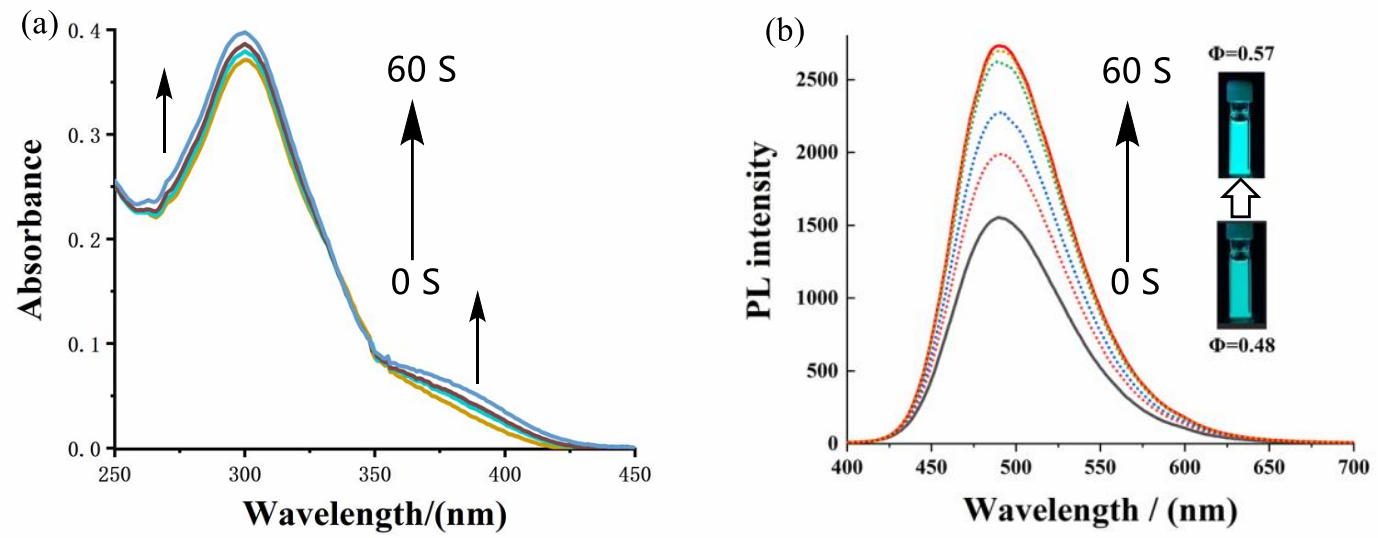

Figure S25. (a) UV-vis absorption and (b) emission changes of 1 in $\mathrm{CHCl}_{3}(c=1.0 \times$ $10^{-5} \mathrm{M}, \lambda_{\mathrm{ex}}=301 \mathrm{~nm}$ ) upon irradiation with $365 \mathrm{~nm}$ UV light at room temperture.
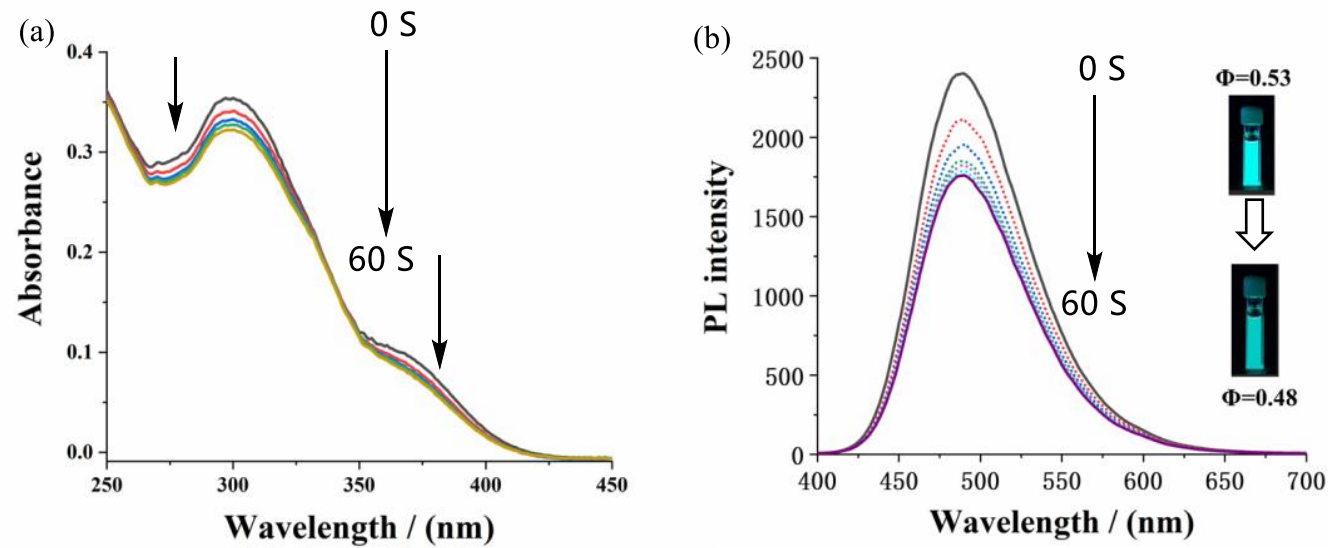

Figure S26. (a) UV-vis absorption and (b) emission changes of 2 in $\mathrm{CHCl}_{3}(c=1.0 \times$ $10^{-5} \mathrm{M}, \lambda_{\mathrm{ex}}=300 \mathrm{~nm}$ ) upon irradiation with $365 \mathrm{~nm}$ UV light at room temperture. 

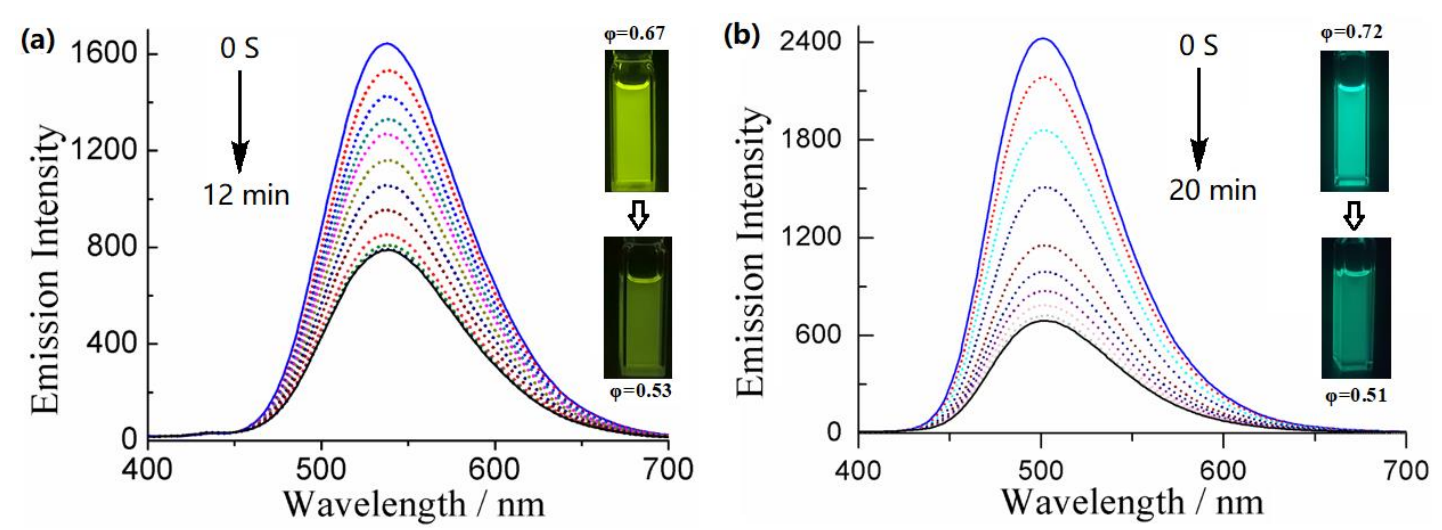

Figure S27. Emission changes of 4 in (a) THF and (b) toluene $\left(c=1.0 \times 10^{-5} \mathrm{M}, \lambda_{\mathrm{ex}}=\right.$ $388 \mathrm{~nm}$ ) upon irradiation with $365 \mathrm{~nm}$ UV light at room temperture.

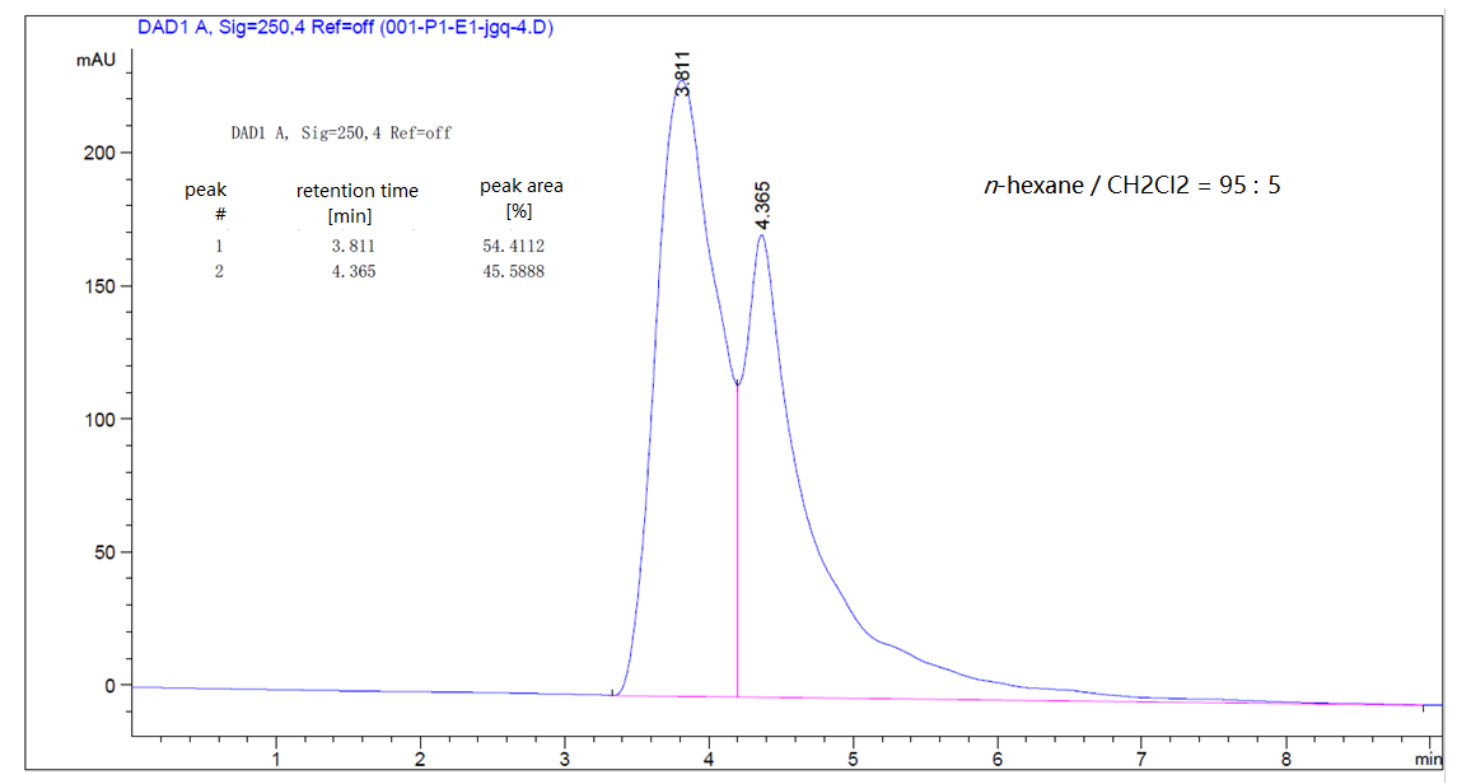

Figure S28. High performance liquid chromatography (HPLC) traces of $\mathbf{4}$ after treatment with UV irradiation. 


\section{Spectroscopic Tracking by ${ }^{1} \mathrm{H}$ NMR Measurements}

\section{1 ${ }^{1} \mathrm{H}$-NMR and ${ }^{11} \mathrm{~B}$-NMR spectral changes of 1}
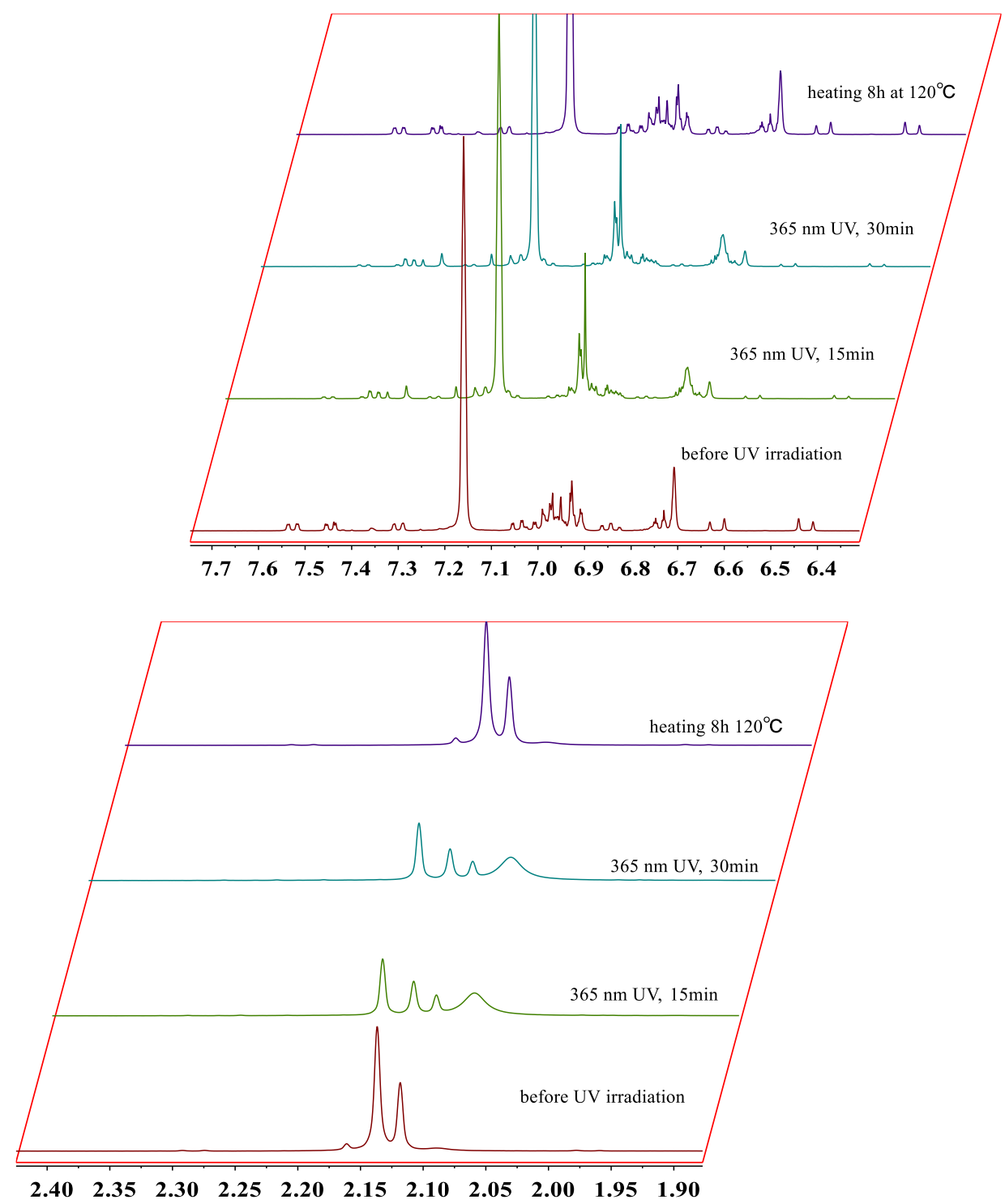

Figure S29. ${ }^{1} \mathrm{H}$ NMR spectral changes of 1 in $\mathrm{C}_{6} \mathrm{D}_{6}$ with $365 \mathrm{~nm}$ UV irradiation at ambient temperature.

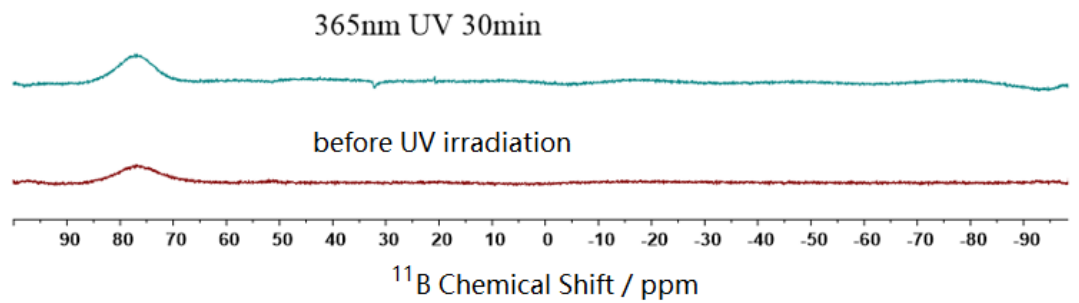

Figure S30. ${ }^{11} \mathrm{~B}$ NMR spectral of compound 1 in $\mathrm{C}_{6} \mathrm{D}_{6}$ before and after with $365 \mathrm{~nm}$ UV irradiation at ambient temperature. 


\section{2 ${ }^{1} \mathrm{H}-\mathrm{NMR}$ and ${ }^{11} \mathrm{~B}-\mathrm{NMR}$ spectral changes of 2}

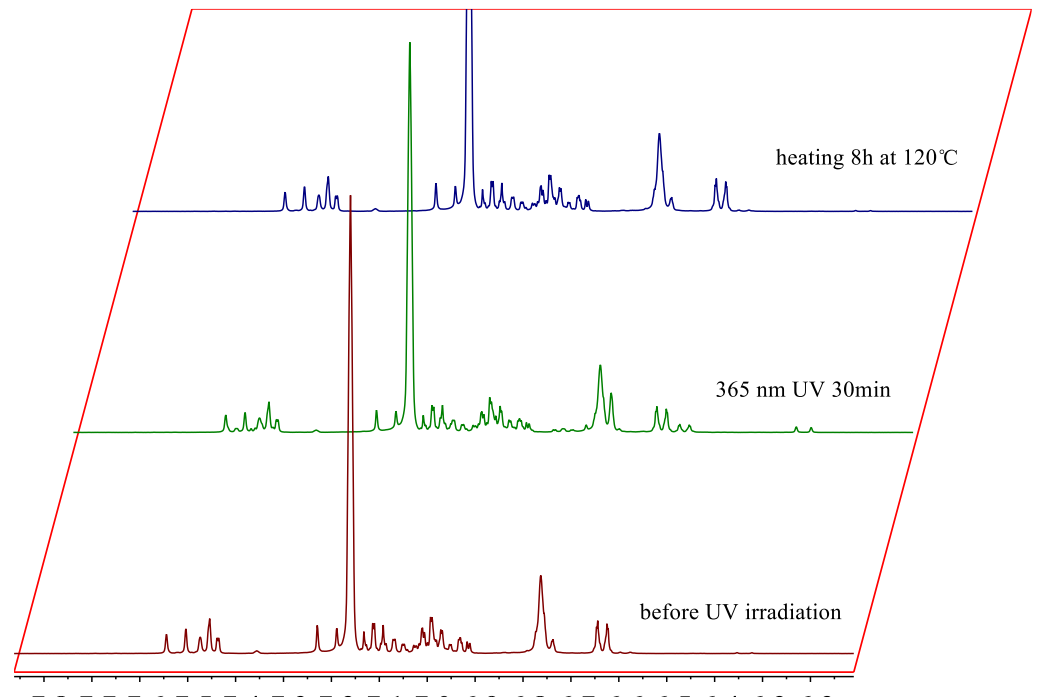

7.8 7.7 7.6 7.5 7.4 7.3 7.2 7.1 7.0 6.9 6.8 6.7 6.6 6.5 6.4 6.3 6.2

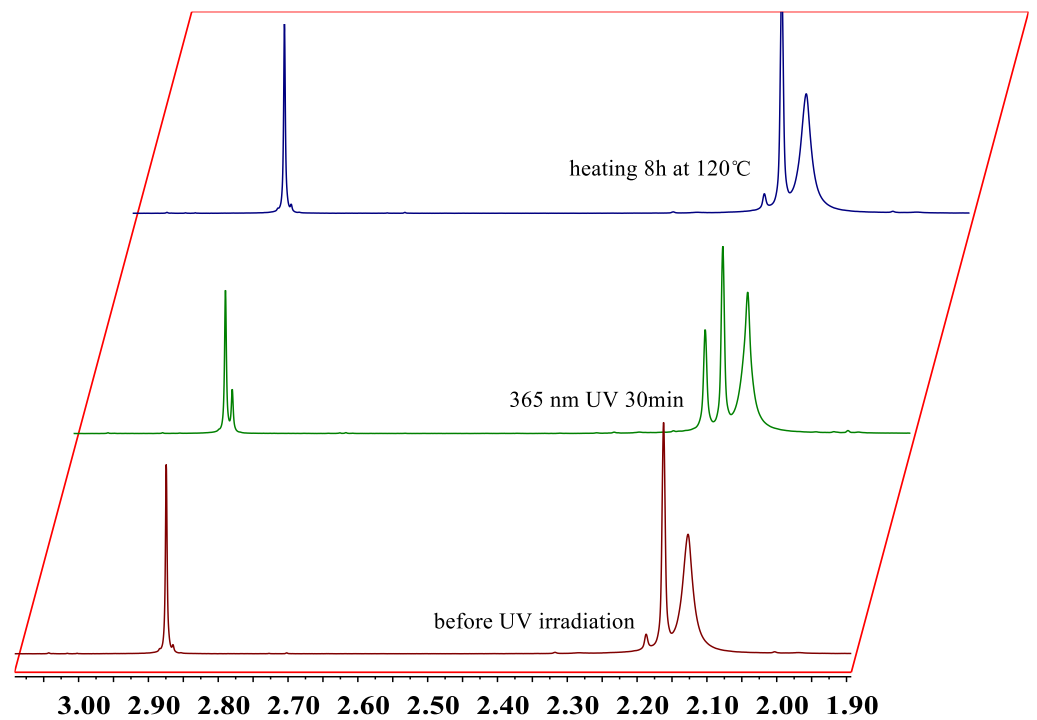

Figure S31. ${ }^{1} \mathrm{H}$ NMR spectral changes of 2 in $\mathrm{C}_{6} \mathrm{D}_{6}$ with $365 \mathrm{~nm}$ UV irradiation at ambient temperature.

$365 \mathrm{~nm} 30 \mathrm{~min}$

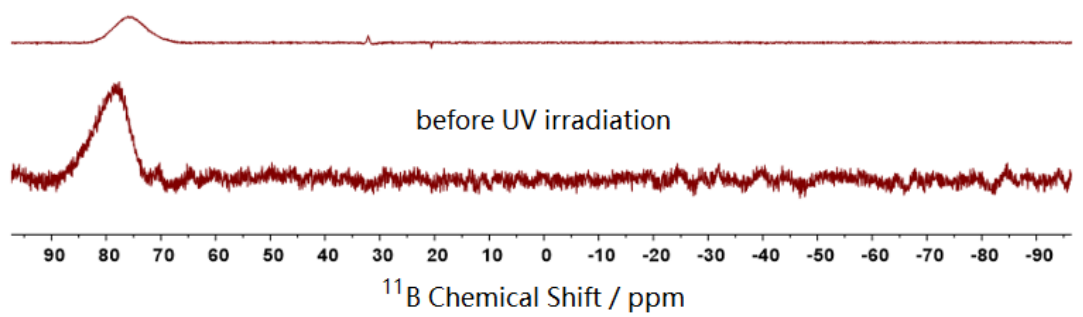

Figure S32. ${ }^{11} \mathrm{~B}$ NMR spectral of 2 in $\mathrm{C}_{6} \mathrm{D}_{6}$ before and after with $365 \mathrm{~nm}$ UV irradiation at ambient temperature. 


\section{$6.3{ }^{1} \mathrm{H}-\mathrm{NMR}$ and ${ }^{11} \mathrm{~B}-\mathrm{NMR}$ spectral changes of 3}
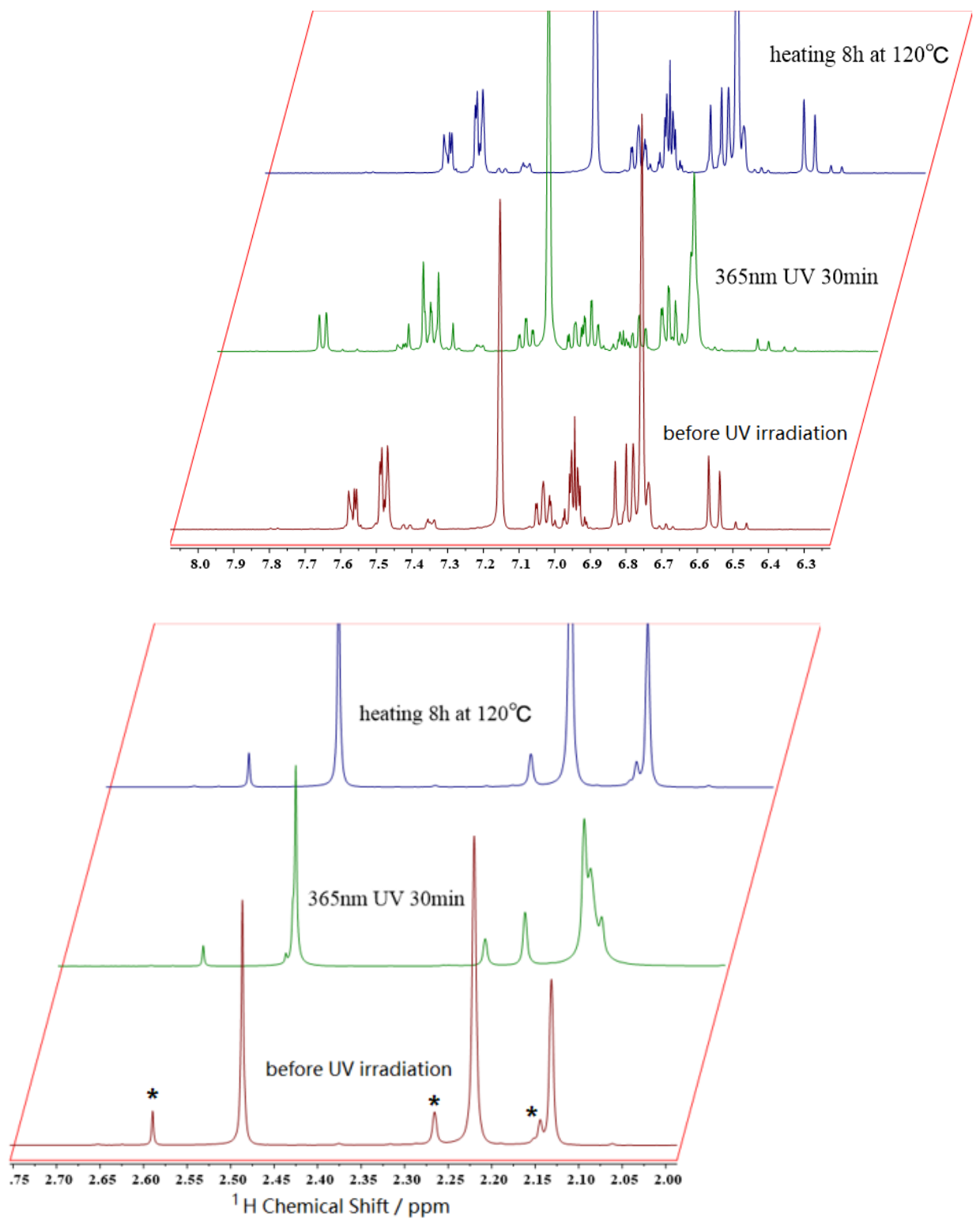

Figure S33. ${ }^{1} \mathrm{H}$ NMR spectral changes of 3 in $\mathrm{C}_{6} \mathrm{D}_{6}$ with $365 \mathrm{~nm}$ UV irradiation at ambient temperature. The asterisk indicated a trace of solvent residue $(\mathrm{N}, \mathrm{N}-$ dimethylacetamide).

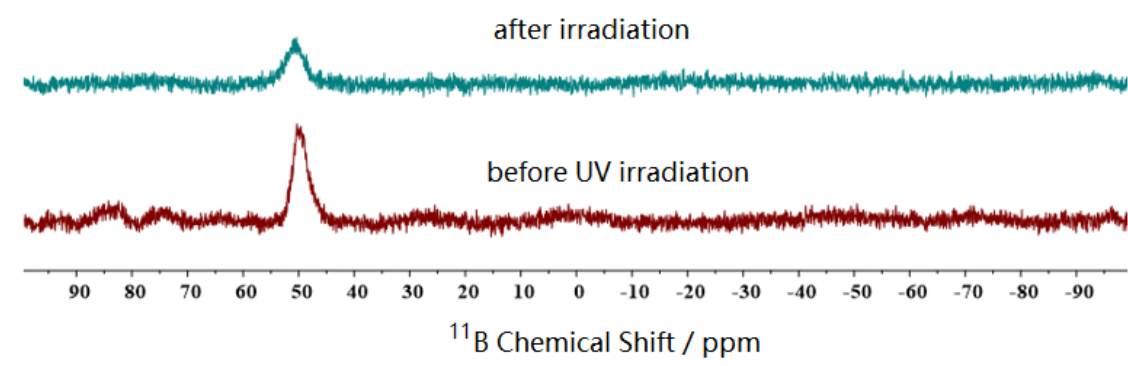

Figure S34. ${ }^{11} \mathrm{~B}$ NMR spectral of 3 in $\mathrm{C}_{6} \mathrm{D}_{6}$ before and after with $365 \mathrm{~nm}$ UV irradiation at ambient temperature. 


\section{$6.4{ }^{1} \mathrm{H}-\mathrm{NMR}$ spectral changes of 4}

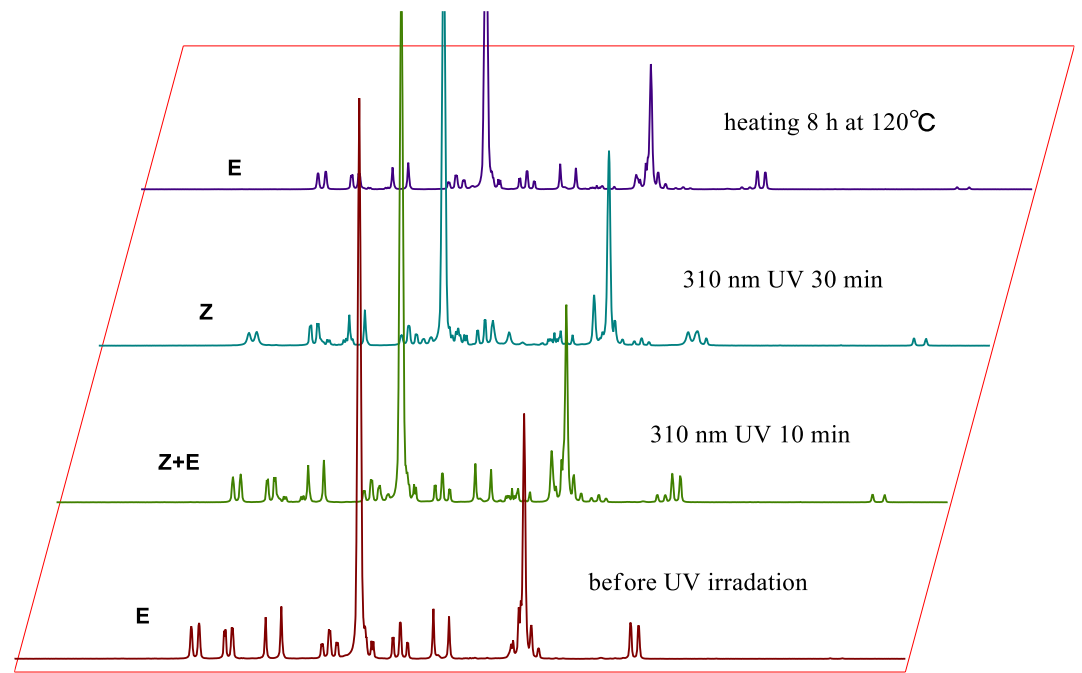

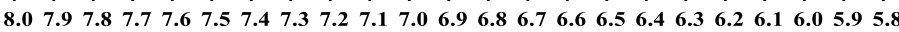

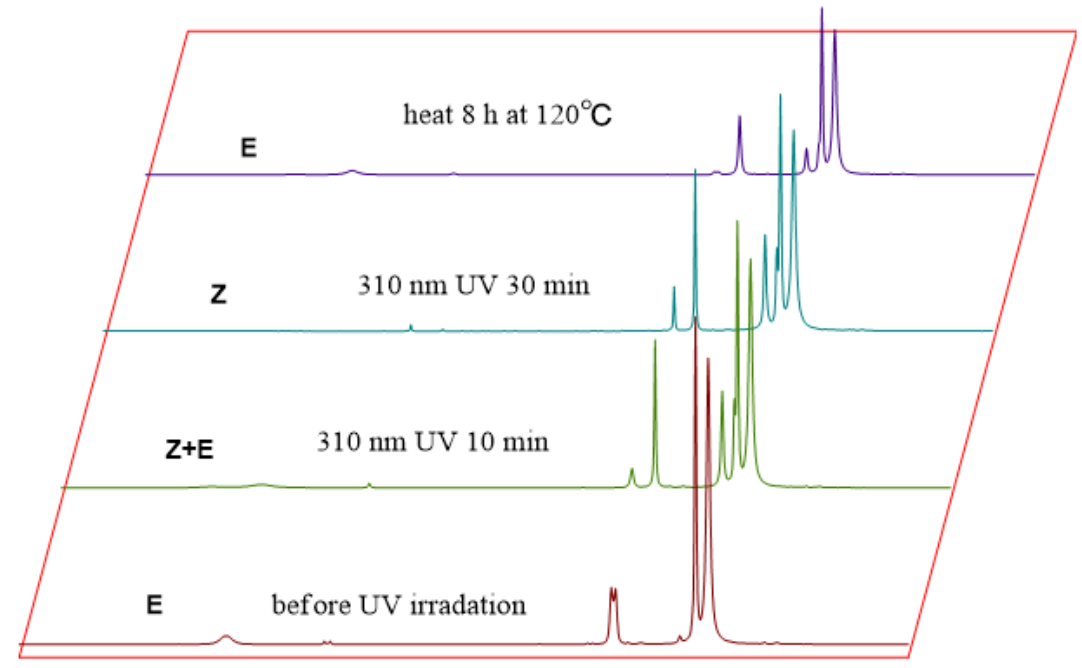

$\begin{array}{llllllllll}3.6 & 3.4 & 3.2 & 3.0 & 2.8 & 2.6 & 2.4 & 2.2 & 2.0 & 1.8\end{array}$

${ }^{1}$ H NMR Chemical Shift / ppm

Figure S35. ${ }^{1} \mathrm{H}$ NMR spectral changes of 4 in $\mathrm{C}_{6} \mathrm{D}_{6}$ with $310 \mathrm{~nm}$ UV irradiation at ambient temperature. 
6.5 Structural Instability Determined by ${ }^{1} \mathrm{H}$ NMR Spectra of the Cis-3

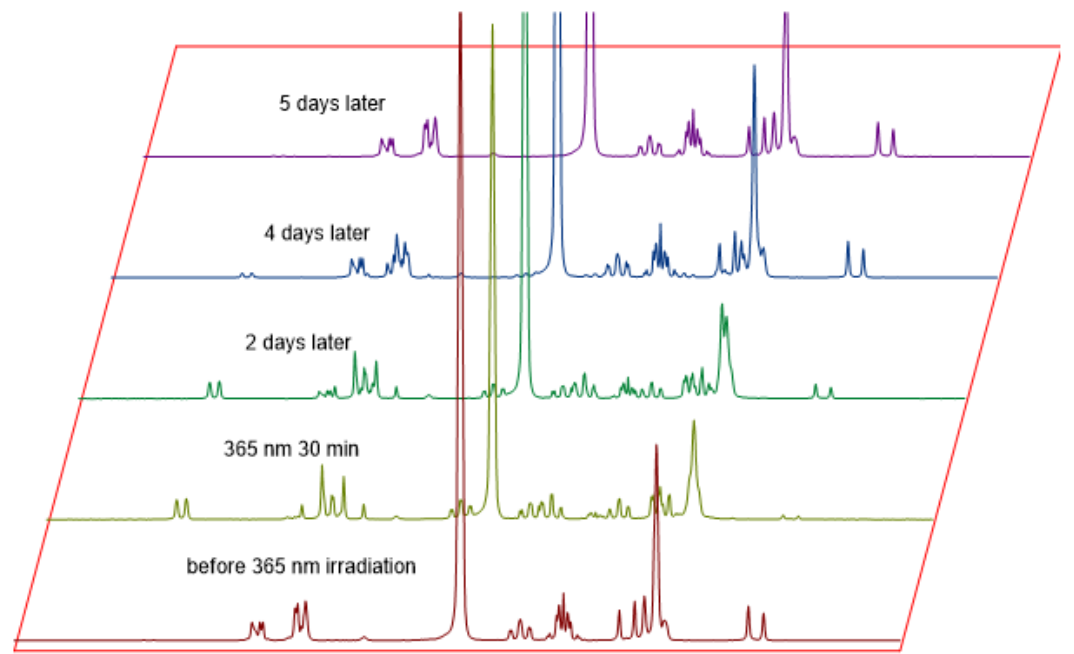

$\begin{array}{llllllllllllllllllllll}8.0 & 7.9 & 7.8 & 7.7 & 7.6 & 7.5 & 7.4 & 7.3 & 7.2 & 7.1 & 7.0 & 6.9 & 6.8 & 6.7 & 6.6 & 6.5 & 6.4 & 6.3\end{array}$

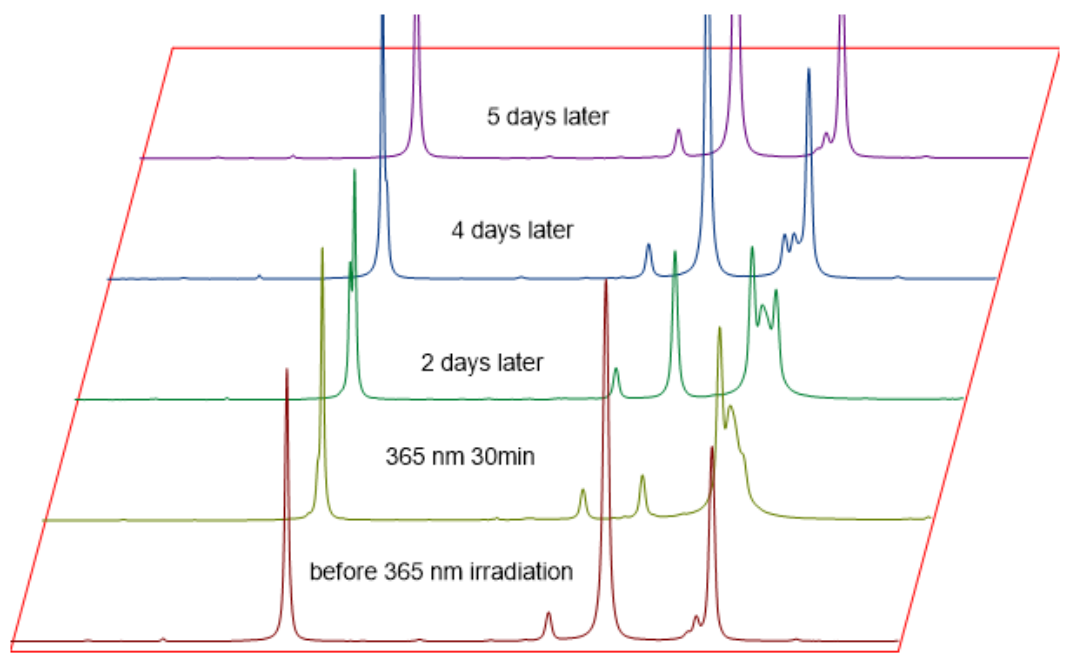

\begin{tabular}{lllllllllllllll}
\hline 2.70 & 2.65 & 2.60 & 2.55 & 2.50 & 2.45 & 2.40 & 2.35 & 2.30 & 2.25 & 2.20 & 2.15 & 2.10 & 2.05 & 2.00
\end{tabular}

${ }^{1} \mathrm{H}$ Chemical Shift / ppm

Figure S36. ${ }^{1} \mathrm{H}$ NMR spectral changes of stock solution 3 after UV irradiation over time (in $\mathrm{C}_{6} \mathrm{D}_{6}$, r.t., under ambient light). 


\subsection{Structural Stability Determined by ${ }^{1} \mathrm{H}$ NMR Spectra of the Cis-4}

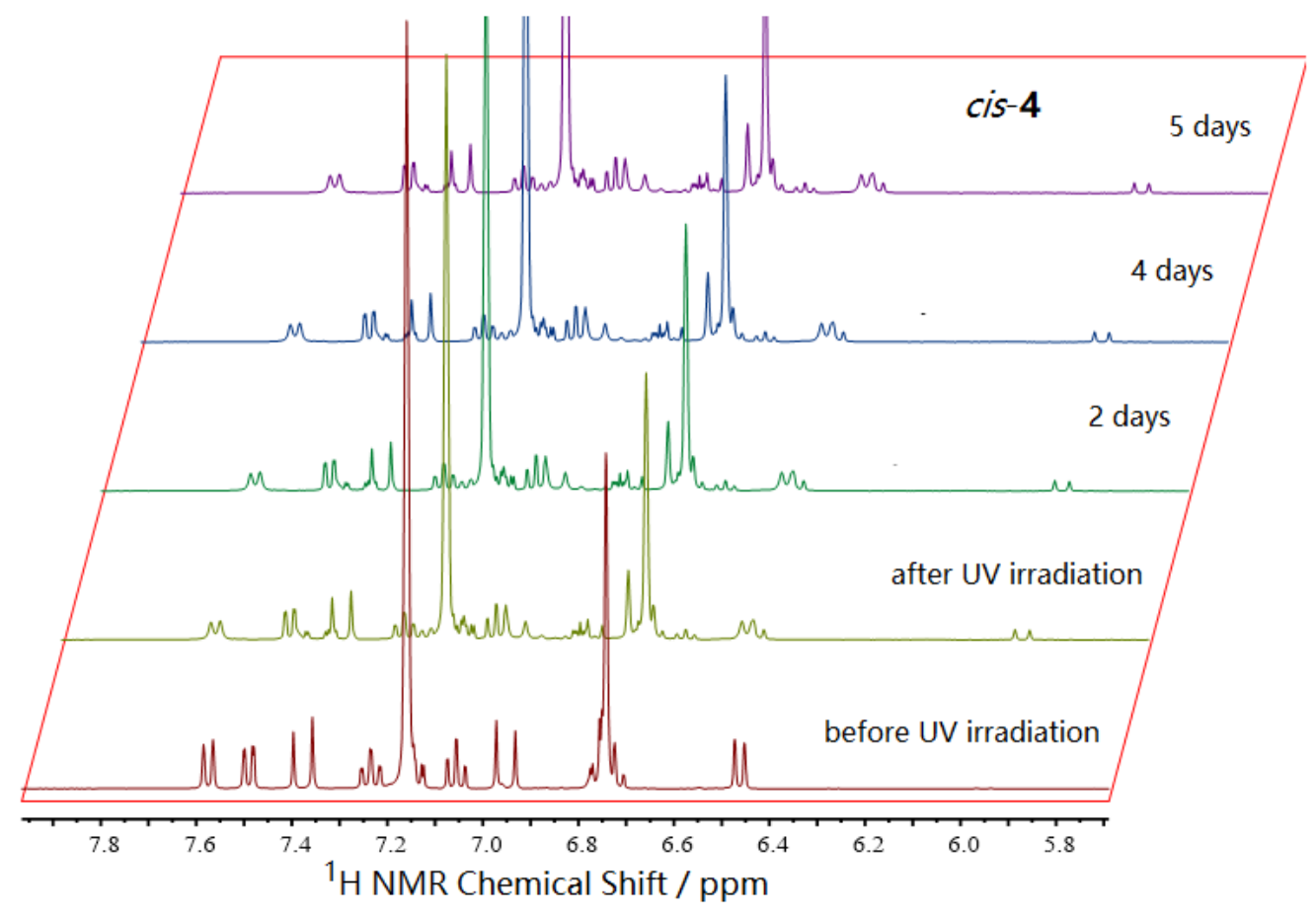

Figure S37. Configurational stability of 4 in the cis form determined by ${ }^{1} \mathrm{H}$ NMR spectra in $\mathrm{C}_{6} \mathrm{D}_{6}$ under ambient light.

\section{DFT and TD-DFT Computations}

DFT and TD-DFT calculations were performed using the Gaussian 09 suite of programs. ${ }^{[1]}$ Geometry optimizations and vertical excitations of all compounds were obtained at the B3LYP/6-31G*, CAM-B3LYP/6-31G* and B3LYP/6-311G** level of theory, ${ }^{[2,3]}$ and the resulting structures were confirmed to be stationary points through vibrational frequency analysis.

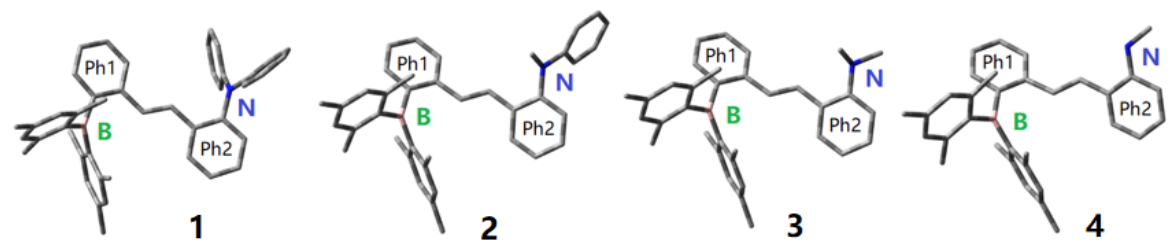

\begin{tabular}{|c|c|c|c|c|}
\hline $\begin{array}{c}\text { Dihedral Angle / } \\
(\mathrm{Ph} 1 \mathrm{vs} \mathrm{Ph} 2)\end{array}$ & 30.8 & 42.3 & 45.1 & 56.1 \\
\hline $\begin{array}{c}\text { Distance } / \AA \\
(\mathrm{B} \ldots \mathrm{N})\end{array}$ & 7.258 & 7.108 & 7.078 & 6.931 \\
\hline
\end{tabular}

Figure S38. DFT optimized structures of 1-4 (B3LYP/6-31G*). Hydrogen atoms are omitted. Calculated dihedral angle $\left(^{\circ}\right)$ for $\mathrm{Ph} 1$ and $\mathrm{Ph} 2$ rings and the distance $(\AA)$ of $\mathrm{B}$ to $\mathrm{N}$ atoms are listed. 


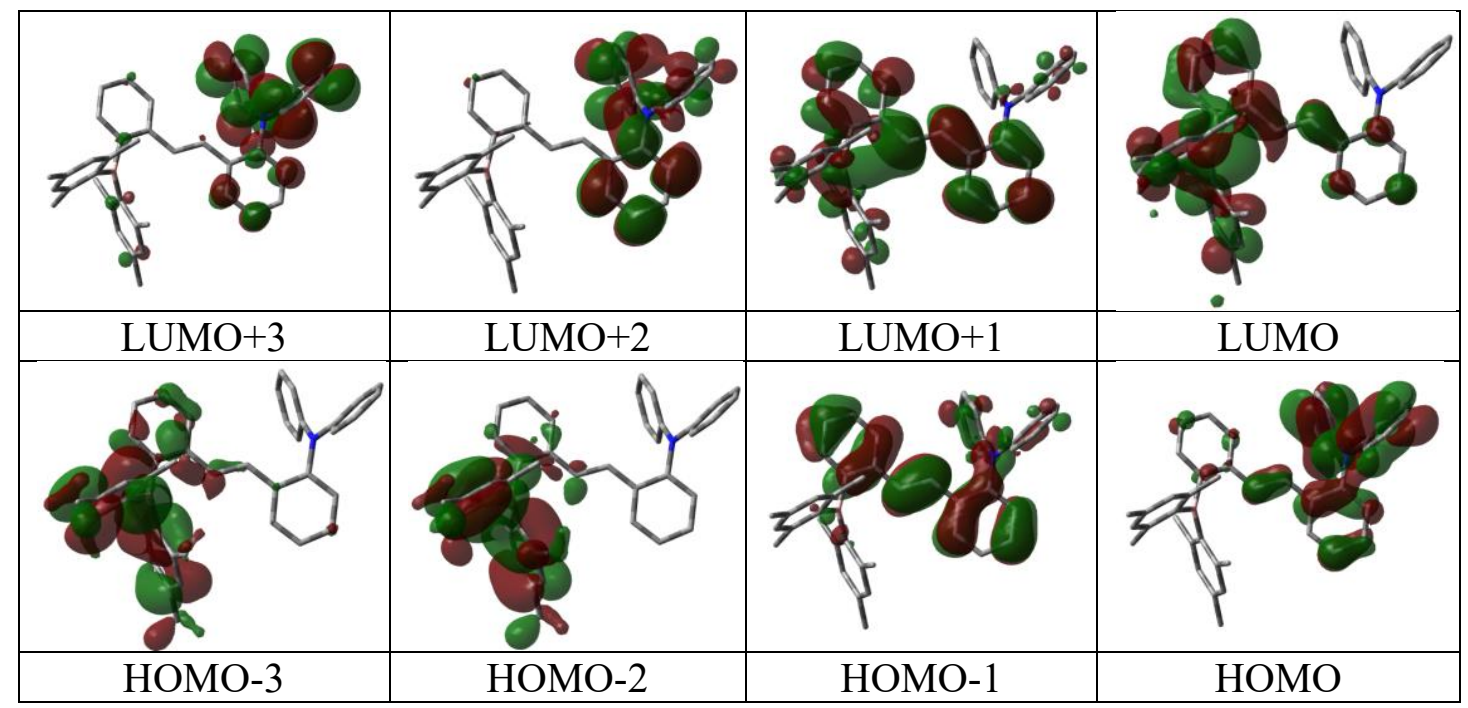

Figure S39. Molecular orbitals contributing to the DFT calculated transitions of 1 (iso $=0.02, \mathrm{~B} 3 \mathrm{LYP} / 6-31 \mathrm{G}^{*}, E=-1781.614723 \mathrm{a} . \mathrm{u}$ ).

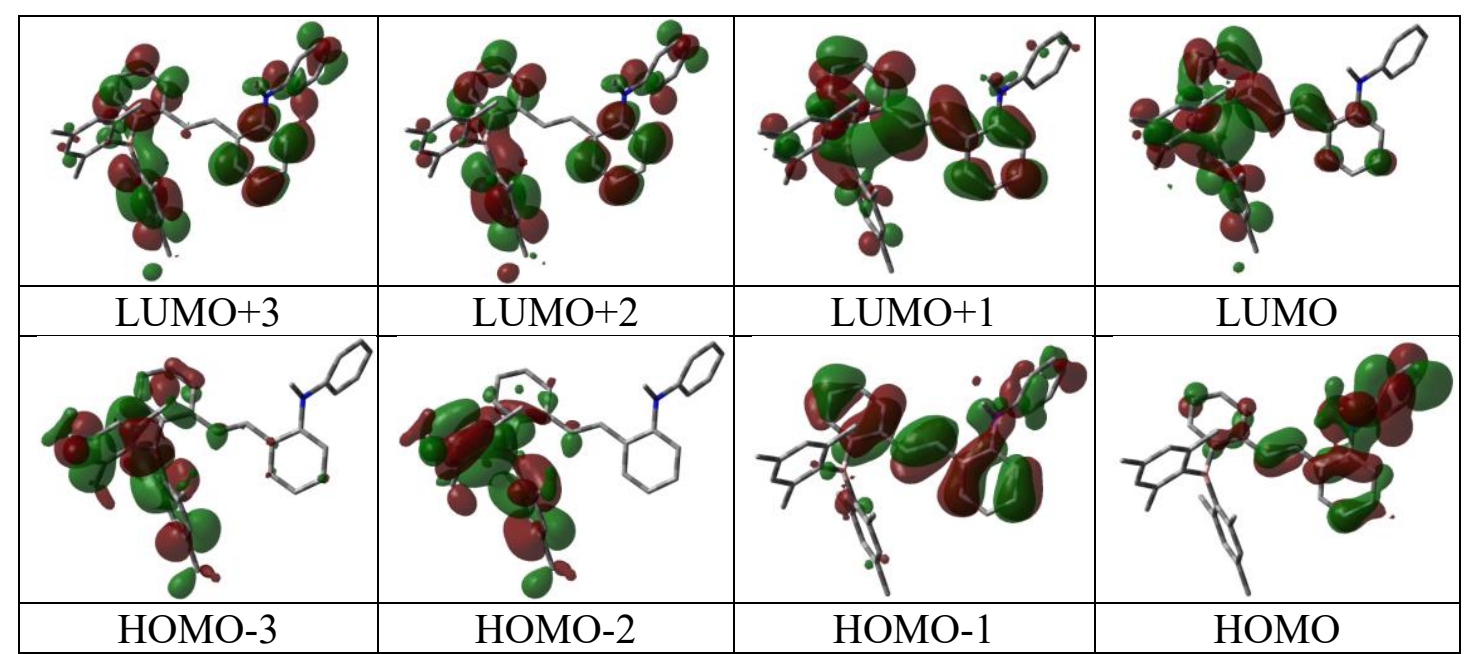

Figure S40. Molecular orbitals contributing to the DFT calculated transitions of 2 (iso $=0.02, \mathrm{~B} 3 \mathrm{LYP} / 6-31 \mathrm{G}^{*}, E=-1589.875111$ a.u). $E_{\text {cis }}=-1859.866979$ a.u. 


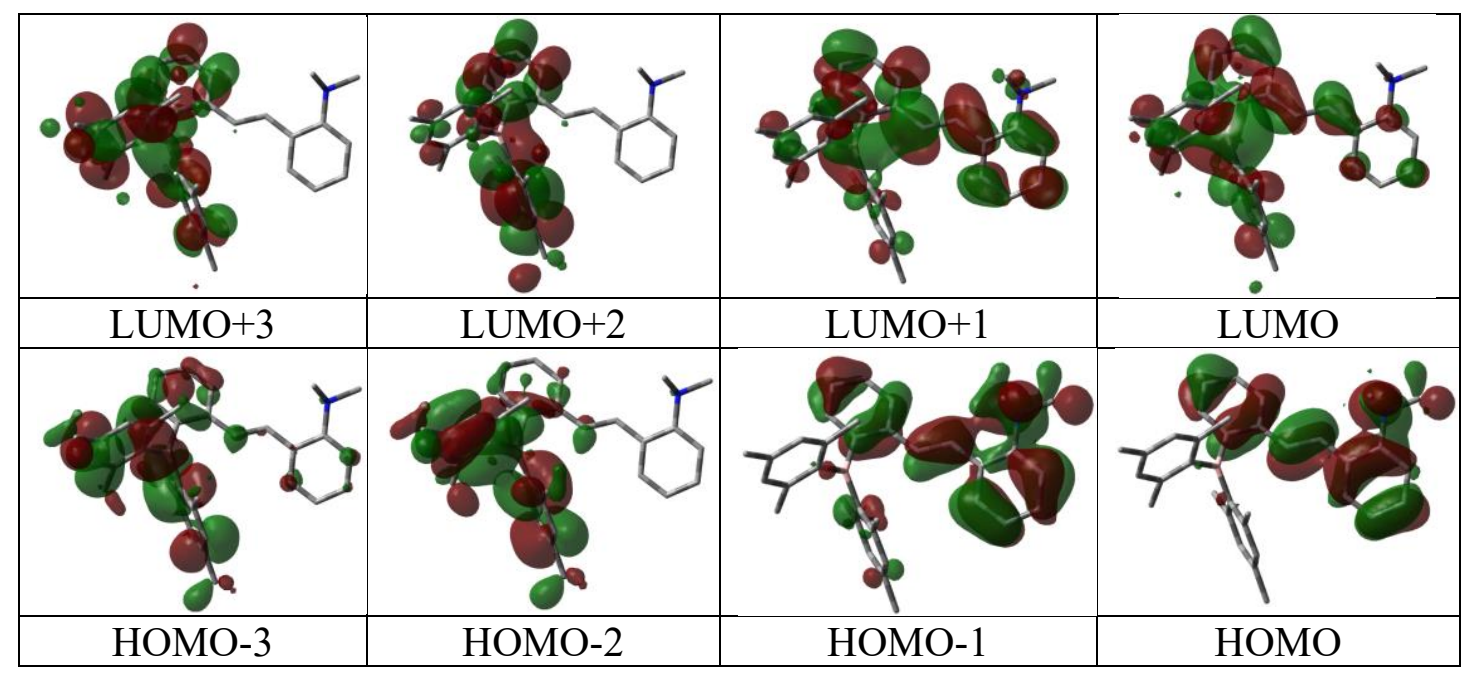

Figure S41. Molecular orbitals contributing to the DFT calculated transitions of 3 (iso $=0.02, \mathrm{~B} 3 \mathrm{LYP} / 6-31 \mathrm{G}^{*}, E=-1398.132422$ a.u). $E_{\mathrm{cis}}=-1398.124918$ a.u.

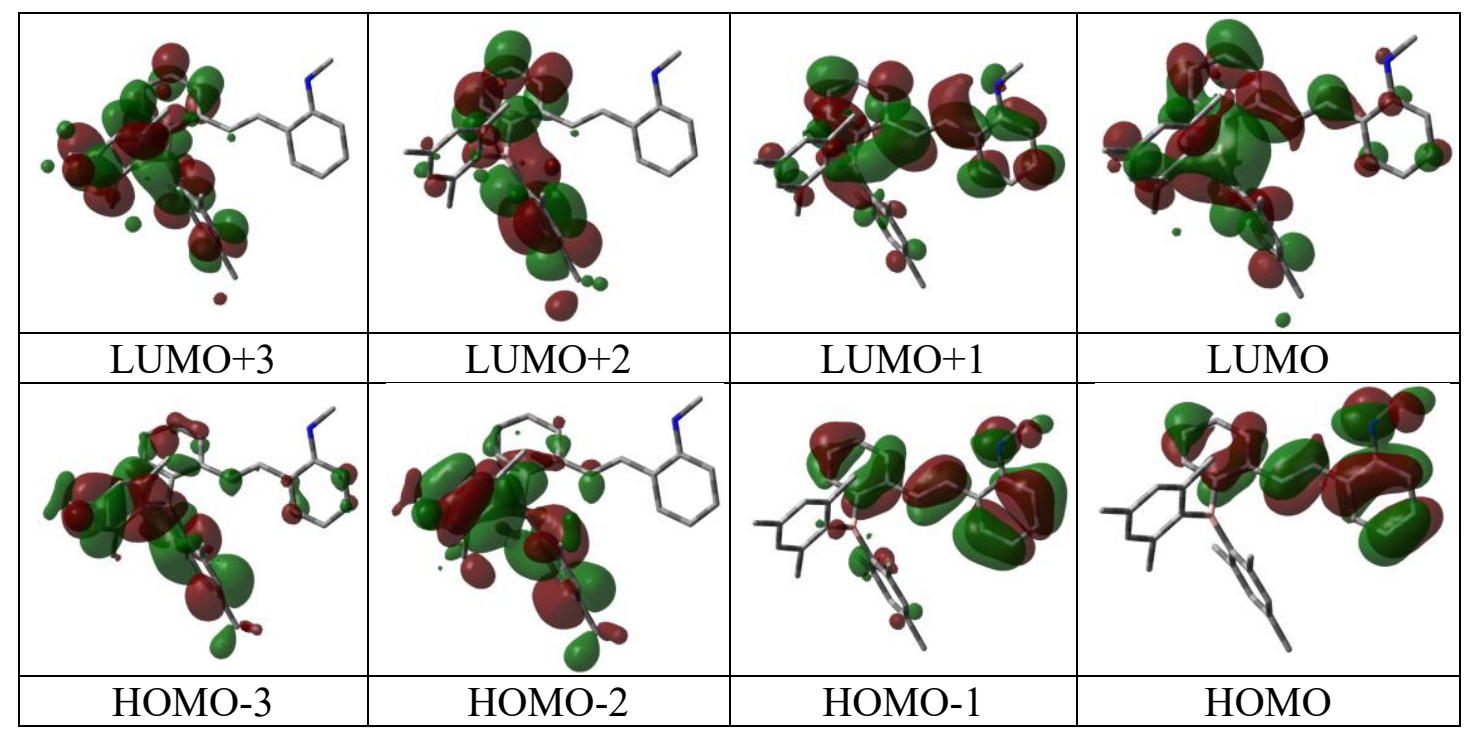

Figure S42. Molecular orbitals contributing to the DFT calculated transitions of 4 (iso $=0.02, \mathrm{~B} 3 \mathrm{LYP} / 6-31 \mathrm{G}^{*}, E=-1358.828954$ a.u). $E_{\mathrm{cis}}=-1358.813335$ a.u.

Table S5. Summary of the DFT calculations for $\mathbf{1 - 4}$.

\begin{tabular}{|c|c|c|c|c|c|c|}
\hline & $\mathrm{E}_{\text {номо }}{ }^{(}(\mathrm{eV})$ & $\mathrm{E}_{\mathrm{Lumo}^{a}}(\mathrm{eV})$ & $\mathrm{E}_{\mathrm{gap}}{ }^{b} / \mathrm{CAM}(\mathrm{eV})$ & $\mathrm{Egap}^{c}(\mathrm{eV})$ & $\mathrm{E}_{\mathrm{TDDFT}^{d}}(\mathrm{eV})$ & $\mathrm{E}_{\mathrm{gap}(\mathrm{opt}}{ }^{e}(\mathrm{eV})$ \\
\hline $\mathbf{1}$ & -6.28 & -0.39 & 5.89 & 3.34 & 2.87 & 2.95 \\
\hline $\mathbf{2}$ & -6.40 & -0.49 & 5.91 & 3.35 & 2.89 & 2.97 \\
\hline $\mathbf{3}$ & -6.52 & -0.41 & 6.11 & 3.52 & 2.98 & 3.01 \\
\hline $\mathbf{4}$ & -6.36 & -0.40 & 5.96 & 3.40 & 2.88 & 2.76 \\
\hline
\end{tabular}

${ }^{a}$ Obtained by DFT calculation (CAM-B3LYP, 6-31 $\left.\mathrm{G}^{*}\right) \cdot{ }^{b}$ HOMO-LUMO energy gap: $\mathrm{E}_{\text {gap }}=\mathrm{E}_{\text {LUMO }}$ $-\mathrm{E}_{\text {номо }}{ }^{c} \mathrm{E}_{\text {gap }}=\mathrm{E}_{\text {LUMO }}-\mathrm{E}_{\text {Hомо }}$ (B3LYP, 6-31G*). ${ }^{d}$ Vertical excitation of the lowest transition ( $\mathrm{S}_{0}$ $\rightarrow \mathrm{S}_{1}$ ) calculated by TD-DFT (B3LYP, 6-311 $\mathrm{G}^{* *}$ ). ${ }^{e}$ Obtained from the absorption onsets in THF. 
Table S6. Comparison of the TD-DFT calculations (B3LYP, 6-311G**) for 1-4.

\begin{tabular}{|c|c|c|c|c|}
\hline Compound & Transition & $\lambda, \mathrm{nm}(\mathrm{eV})$ & $\begin{array}{l}\text { Oscillator } \\
\text { Strength, } f\end{array}$ & Orbital Contributions \\
\hline \multirow{5}{*}{1} & $\mathrm{~S}_{0} \rightarrow \mathrm{S}_{1}$ & $432(2.87)$ & 0.0778 & $\mathrm{HOMO} \rightarrow$ LUMO $(91 \%)$ \\
\hline & $\mathrm{S}_{0} \longrightarrow \mathrm{S}_{2}$ & $391(3.17)$ & 0.0073 & $\begin{array}{l}\mathrm{HOMO}-1 \rightarrow \text { LUMO }(13 \%) \\
\mathrm{HOMO} \rightarrow \mathrm{LUMO}+1(81 \%)\end{array}$ \\
\hline & $\mathrm{S}_{0} \rightarrow \mathrm{S}_{3}$ & $370(3.35)$ & 0.1449 & HOMO-1 $\rightarrow$ LUMO (80\%) \\
\hline & $\mathrm{S}_{0} \rightarrow \mathrm{S}_{4}$ & $348(3.56)$ & 0.0814 & HOMO-2 $\rightarrow$ LUMO $(95 \%)$ \\
\hline & $\mathrm{S}_{0} \rightarrow \mathrm{S}_{5}$ & $329(3.76)$ & 0.1096 & $\begin{array}{l}\text { HOMO-3 } \rightarrow \text { LUMO }(70 \%) \\
\text { HOMO-1 } \rightarrow \text { LUMO+1 }(22 \%)\end{array}$ \\
\hline \multirow{5}{*}{2} & $\mathrm{~S}_{0} \rightarrow \mathrm{S}_{1}$ & $429(2.89)$ & 0.1274 & $\mathrm{HOMO} \rightarrow$ LUMO $(95 \%)$ \\
\hline & $\mathrm{S}_{0} \rightarrow \mathrm{S}_{2}$ & $382(3.25)$ & 0.0019 & $\begin{array}{l}\text { HOMO-1 } \rightarrow \text { LUMO }(51 \%) \\
\text { HOMO } \rightarrow \text { LUMO+1 }(47 \%)\end{array}$ \\
\hline & $\mathrm{S}_{0} \rightarrow \mathrm{S}_{3}$ & $366(3.38)$ & 0.1922 & $\begin{array}{l}\text { HOMO- } \rightarrow \text { LUMO }(40 \%) \\
\text { HOMO } \rightarrow \text { LUMO+1 }(49 \%)\end{array}$ \\
\hline & $\mathrm{S}_{0} \rightarrow \mathrm{S}_{4}$ & $350(3.54)$ & 0.1094 & HOMO-2 $\rightarrow$ LUMO $(93 \%)$ \\
\hline & $\mathrm{S}_{0} \rightarrow \mathrm{S}_{5}$ & $330(3.76)$ & 0.0334 & $\begin{array}{l}\text { HOMO-3 } \rightarrow \text { LUMO }(83 \%) \\
\text { HOMO-1 } \rightarrow \text { LUMO+1 }(11 \%)\end{array}$ \\
\hline \multirow{5}{*}{3} & $\mathrm{~S}_{0} \rightarrow \mathrm{S}_{1}$ & $416(2.98)$ & 0.1524 & $\mathrm{HOMO} \rightarrow \mathrm{LUMO}(96 \%)$ \\
\hline & $\mathrm{S}_{0} \rightarrow \mathrm{S}_{2}$ & $356(3.48)$ & 0.0567 & $\begin{array}{l}\text { HOMO-1 } \rightarrow \text { LUMO }(60 \%) \\
\text { HOMO } \rightarrow \text { LUMO+1 }(26 \%)\end{array}$ \\
\hline & $\mathrm{S}_{0} \rightarrow \mathrm{S}_{3}$ & $351(3.54)$ & 0.0471 & $\begin{array}{l}\mathrm{HOMO}-2 \rightarrow \text { LUMO }(77 \%) \\
\mathrm{HOMO} \rightarrow \mathrm{LUMO}+1(19 \%)\end{array}$ \\
\hline & $\mathrm{S}_{0} \rightarrow \mathrm{S}_{4}$ & $339(3.65)$ & 0.2645 & $\begin{array}{l}\text { HOMO-3 } \rightarrow \text { LUMO }(23 \%) \\
\text { HOMO-1 } \rightarrow \text { LUMO }(26 \%) \\
\text { HOMO } \rightarrow \text { LUMO+1 }(35 \%)\end{array}$ \\
\hline & $\mathrm{S}_{0} \rightarrow \mathrm{S}_{5}$ & $327(3.79)$ & 0.0483 & $\begin{array}{l}\text { HOMO-3 } \rightarrow \text { LUMO }(51 \%) \\
\text { HOMO-4 } \rightarrow \text { LUMO }(33 \%)\end{array}$ \\
\hline \multirow{5}{*}{4} & $\mathrm{~S}_{0} \rightarrow \mathrm{S}_{1}$ & $430(2.88)$ & 0.1189 & $\mathrm{HOMO} \rightarrow \mathrm{LUMO}(97 \%)$ \\
\hline & $\mathrm{S}_{0} \rightarrow \mathrm{S}_{2}$ & $361(3.43)$ & 0.1951 & $\begin{array}{l}\text { HOMO-1 } \rightarrow \text { LUMO (12\%) } \\
\mathrm{HOMO} \rightarrow \mathrm{LUMO}+1(83 \%)\end{array}$ \\
\hline & $\mathrm{S}_{0} \rightarrow \mathrm{S}_{3}$ & $351(3.53)$ & 0.0482 & $\begin{array}{l}\text { HOMO- }- \text { LUMO }(75 \%) \\
\text { HOMO- } 1 \rightarrow \text { LUMO }(20 \%)\end{array}$ \\
\hline & $\mathrm{S}_{0} \rightarrow \mathrm{S}_{4}$ & $342(3.62)$ & 0.1867 & $\begin{array}{l}\text { HOMO-2 } \rightarrow \text { LUMO }(21 \%) \\
\text { HOMO- } 1 \rightarrow \text { LUMO }(56 \%)\end{array}$ \\
\hline & $\mathrm{S}_{0} \rightarrow \mathrm{S}_{5}$ & $324(3.83)$ & 0.2517 & HOMO-3 $\rightarrow$ LUMO $(80 \%)$ \\
\hline
\end{tabular}

Table S7. Coordinates $(\AA ̊)$ for the optimized structure (B3LYP, 6-31G*) of $\mathbf{1}$.

\begin{tabular}{|c|c|c|c|c|c|c|c|}
\hline atom & $\mathrm{X}$ & $\mathrm{Y}$ & $\mathrm{Z}$ & atom & $\mathrm{X}$ & $\mathrm{Y}$ & $\mathrm{Z}$ \\
\hline $\mathrm{C}$ & 4.129662 & 1.620813 & -2.116377 & $\mathrm{H}$ & 5.193608 & 1.472079 & -2.275133 \\
\hline $\mathrm{C}$ & 3.490927 & 0.874952 & -1.116933 & $\mathrm{H}$ & 0.347233 & 2.1241 & -1.51358 \\
\hline $\mathrm{C}$ & 2.100591 & 1.032093 & -0.881052 & $\mathrm{H}$ & 1.488838 & 3.436125 & -3.241231 \\
\hline $\mathrm{C}$ & 1.41263 & 1.980436 & -1.665729 & $\mathrm{H}$ & 3.930204 & 3.109248 & -3.656943 \\
\hline $\mathrm{C}$ & 2.054811 & 2.720537 & -2.651004 & $\mathrm{H}$ & 2.031259 & -0.59204 & 0.542813 \\
\hline
\end{tabular}




\begin{tabular}{|c|c|c|c|c|c|c|c|}
\hline $\mathrm{C}$ & 3.420589 & 2.536414 & -2.887135 & $\mathrm{H}$ & -0.489075 & 1.087931 & 0.095575 \\
\hline $\mathrm{C}$ & 1.424046 & 0.203268 & 0.122548 & $\mathrm{H}$ & 1.342885 & -0.889105 & 2.61057 \\
\hline $\mathrm{C}$ & 0.144708 & 0.32476 & 0.534612 & $\mathrm{H}$ & -3.479862 & -1.95152 & 2.406261 \\
\hline $\mathrm{C}$ & -0.498547 & -0.530021 & 1.545304 & $\mathrm{H}$ & 0.351055 & -2.401339 & 4.281322 \\
\hline $\mathrm{C}$ & -1.89984 & -0.802398 & 1.497964 & $\mathrm{H}$ & -2.086804 & -2.934973 & 4.187625 \\
\hline $\mathrm{C}$ & 0.283545 & -1.122014 & 2.555663 & $\mathrm{H}$ & 6.814581 & -0.834385 & 0.094076 \\
\hline $\mathrm{C}$ & -2.42326 & -1.700342 & 2.45263 & $\mathrm{H}$ & 3.342039 & -1.520847 & -2.346464 \\
\hline $\mathrm{C}$ & -0.276692 & -1.972022 & 3.504463 & $\mathrm{H}$ & 8.029153 & -2.594481 & -1.138127 \\
\hline $\mathrm{C}$ & -1.640128 & -2.267101 & 3.455645 & $\mathrm{H}$ & 4.567 & -3.262877 & -3.600257 \\
\hline $\mathrm{C}$ & 4.989207 & -1.060005 & -1.034846 & $\mathrm{H}$ & 6.919658 & -3.824467 & -2.999483 \\
\hline $\mathrm{C}$ & 6.319162 & -1.372843 & -0.707095 & $\mathrm{H}$ & 4.688844 & -1.867574 & 1.573053 \\
\hline $\mathrm{C}$ & 4.369522 & -1.755373 & -2.086866 & $\mathrm{H}$ & 4.343475 & 2.356513 & 0.839413 \\
\hline $\mathrm{C}$ & 7.000482 & -2.36759 & -1.406645 & $\mathrm{H}$ & 5.049993 & -1.421082 & 3.972383 \\
\hline $\mathrm{C}$ & 5.065131 & -2.736073 & -2.790365 & $\mathrm{H}$ & 4.675321 & 2.800208 & 3.243138 \\
\hline $\mathrm{C}$ & 6.3825 & -3.054165 & -2.453539 & $\mathrm{H}$ & 5.041875 & 0.918482 & 4.835253 \\
\hline $\mathrm{C}$ & 4.499219 & 0.21419 & 1.032032 & $\mathrm{H}$ & -7.447687 & -1.491902 & -0.366099 \\
\hline $\mathrm{C}$ & 4.695486 & -0.845298 & 1.937561 & $\mathrm{H}$ & -4.377407 & -4.118587 & -1.776978 \\
\hline $\mathrm{C}$ & 4.49564 & 1.529244 & 1.524506 & $\mathrm{H}$ & -2.927106 & 3.45093 & -2.599903 \\
\hline $\mathrm{C}$ & 4.897762 & -0.587462 & 3.291346 & $\mathrm{H}$ & -2.919205 & 4.459172 & 1.559413 \\
\hline $\mathrm{C}$ & 4.682945 & 1.774052 & 2.884616 & $\mathrm{H}$ & -5.526894 & 0.520597 & 1.714446 \\
\hline $\mathrm{C}$ & 4.890606 & 0.722592 & 3.777662 & $\mathrm{H}$ & -5.663426 & 1.311741 & 0.148942 \\
\hline $\mathrm{C}$ & -4.051318 & -1.15147 & -0.099097 & $\mathrm{H}$ & -7.026647 & 0.386706 & 0.794964 \\
\hline $\mathrm{C}$ & -5.435077 & -0.848253 & 0.016784 & $\mathrm{H}$ & -1.58596 & -1.968883 & -1.211106 \\
\hline $\mathrm{C}$ & -3.690096 & -2.364284 & -0.747654 & $\mathrm{H}$ & -1.836458 & -3.220636 & -0.004559 \\
\hline $\mathrm{C}$ & -6.393206 & -1.734644 & -0.486166 & $\mathrm{H}$ & -2.169441 & -3.559894 & -1.707915 \\
\hline $\mathrm{C}$ & -4.679169 & -3.205455 & -1.266346 & $\mathrm{H}$ & -3.886963 & 0.229892 & -2.442629 \\
\hline $\mathrm{C}$ & -6.039324 & -2.91483 & -1.143031 & $\mathrm{H}$ & -2.843701 & 1.330452 & -3.355139 \\
\hline $\mathrm{C}$ & -2.926159 & 1.356979 & 0.11054 & $\mathrm{H}$ & -2.137534 & 0.075402 & -2.335402 \\
\hline C & -2.947274 & 1.783909 & -1.245827 & $\mathrm{H}$ & -8.030895 & -3.76686 & -1.168713 \\
\hline $\mathrm{C}$ & -2.901413 & 2.357872 & 1.12015 & $\mathrm{H}$ & -7.310994 & -3.569716 & -2.768728 \\
\hline C & -2.929953 & 3.148611 & -1.553924 & $\mathrm{H}$ & -6.759854 & -4.875753 & -1.716719 \\
\hline $\mathrm{C}$ & -2.920246 & 3.711465 & 0.768083 & $\mathrm{H}$ & -3.574029 & 1.23645 & 2.874174 \\
\hline $\mathrm{C}$ & -2.929841 & 4.132954 & -0.562734 & $\mathrm{H}$ & -1.874387 & 1.676796 & 2.911629 \\
\hline $\mathrm{C}$ & -5.935843 & 0.408169 & 0.703751 & $\mathrm{H}$ & -3.108512 & 2.909845 & 3.197236 \\
\hline $\mathrm{C}$ & -2.24568 & -2.795162 & -0.927761 & $\mathrm{H}$ & -4.000204 & 5.968381 & -0.977453 \\
\hline $\mathrm{C}$ & -2.955076 & 0.802598 & -2.402478 & $\mathrm{H}$ & -2.450795 & 6.207978 & -0.165886 \\
\hline $\mathrm{C}$ & -7.089527 & -3.831104 & -1.724933 & $\mathrm{H}$ & -2.498714 & 5.79108 & -1.888928 \\
\hline $\mathrm{C}$ & -2.865528 & 2.024738 & 2.600242 & B & -2.938625 & -0.184259 & 0.482348 \\
\hline $\mathrm{C}$ & -2.966858 & 5.600466 & -0.917226 & $\mathrm{~N}$ & 4.270567 & -0.047301 & -0.343527 \\
\hline
\end{tabular}

Table S8. Coordinates $(\AA)$ for the optimized structure (B3LYP, 6-31G*) of 2.

\begin{tabular}{|c|c|c|c|c|c|c|c|}
\hline atom & $\mathrm{X}$ & $\mathrm{Y}$ & $\mathrm{Z}$ & atom & $\mathrm{X}$ & $\mathrm{Y}$ & $\mathrm{Z}$ \\
\hline $\mathrm{C}$ & 4.615114 & 1.547539 & -1.649422 & $\mathrm{H}$ & 5.578353 & 1.273208 & -2.069494 \\
\hline $\mathrm{C}$ & 3.889753 & 0.582323 & -0.938481 & $\mathrm{H}$ & 1.190839 & 2.49544 & -0.123399 \\
\hline $\mathrm{C}$ & 2.630596 & 0.909478 & -0.377269 & $\mathrm{H}$ & 2.463839 & 4.168277 & -1.406522 \\
\hline $\mathrm{C}$ & 2.140473 & 2.216692 & -0.570362 & $\mathrm{H}$ & 4.685843 & 3.571442 & -2.374841 \\
\hline $\mathrm{C}$ & 2.864146 & 3.165666 & -1.282922 & $\mathrm{H}$ & 2.51118 & -0.89628 & 0.797405 \\
\hline $\mathrm{C}$ & 4.112015 & 2.834204 & -1.819796 & $\mathrm{H}$ & -0.03255 & 0.693838 & 0.168157 \\
\hline $\mathrm{C}$ & 1.897046 & -0.087004 & 0.409243 & $\mathrm{H}$ & 1.571898 & -1.749808 & 2.511884 \\
\hline $\mathrm{C}$ & 0.567382 & -0.073178 & 0.647089 & $\mathrm{H}$ & -3.305856 & -2.314819 & 1.962695 \\
\hline $\mathrm{C}$ & -0.179094 & -1.032409 & 1.472322 & $\mathrm{H}$ & 0.383703 & -3.444651 & 3.834995 \\
\hline $\mathrm{C}$ & -1.595582 & -1.163397 & 1.336835 & $\mathrm{H}$ & -2.081355 & -3.739094 & 3.561509 \\
\hline $\mathrm{C}$ & 0.500384 & -1.866708 & 2.381429 & $\mathrm{H}$ & 5.709315 & 0.738511 & 0.948229 \\
\hline $\mathrm{C}$ & -2.234845 & -2.175111 & 2.085329 & $\mathrm{H}$ & 6.08298 & -2.735962 & -1.57739 \\
\hline $\mathrm{C}$ & -0.170064 & -2.824557 & 3.134512 & $\mathrm{H}$ & 7.985574 & 0.35648 & 1.794413 \\
\hline
\end{tabular}




\begin{tabular}{|c|c|c|c|c|c|c|c|}
\hline $\mathrm{C}$ & -1.548837 & -2.986985 & 2.985365 & $\mathrm{H}$ & 8.334383 & -3.12498 & -0.702012 \\
\hline $\mathrm{C}$ & 5.713509 & -0.9643 & -0.382543 & $\mathrm{H}$ & 9.329895 & -1.585556 & 0.98636 \\
\hline $\mathrm{C}$ & 6.28657 & -0.101589 & 0.576349 & $\mathrm{H}$ & -7.144147 & -0.892803 & -0.620679 \\
\hline $\mathrm{C}$ & 6.479807 & -2.055961 & -0.832425 & $\mathrm{H}$ & -4.314944 & -3.429838 & -2.589464 \\
\hline $\mathrm{C}$ & 7.572528 & -0.324403 & 1.054171 & $\mathrm{H}$ & -2.131749 & 3.952127 & -1.744166 \\
\hline $\mathrm{C}$ & 7.766552 & -2.273309 & -0.335263 & $\mathrm{H}$ & -2.045863 & 4.025923 & 2.533756 \\
\hline $\mathrm{C}$ & 8.327164 & -1.413373 & 0.606242 & $\mathrm{H}$ & -5.066748 & 0.418759 & 1.843032 \\
\hline $\mathrm{C}$ & -3.735965 & -0.94973 & -0.30071 & $\mathrm{H}$ & -5.115881 & 1.543085 & 0.490937 \\
\hline $\mathrm{C}$ & -5.086141 & -0.54726 & -0.113895 & $\mathrm{H}$ & -6.564345 & 0.628163 & 0.933803 \\
\hline $\mathrm{C}$ & -3.481396 & -2.019792 & -1.201081 & $\mathrm{H}$ & -1.342044 & -1.739302 & -1.57525 \\
\hline $\mathrm{C}$ & -6.115681 & -1.203644 & -0.79648 & $\mathrm{H}$ & -1.73094 & -3.190279 & -0.664166 \\
\hline $\mathrm{C}$ & -4.536752 & -2.626003 & -1.88928 & $\mathrm{H}$ & -2.063936 & -3.124264 & -2.399809 \\
\hline $\mathrm{C}$ & -5.864783 & -2.238384 & -1.699835 & $\mathrm{H}$ & -3.436989 & 0.904601 & -2.281059 \\
\hline $\mathrm{C}$ & -2.377177 & 1.331003 & 0.447082 & $\mathrm{H}$ & -2.266389 & 2.056536 & -2.941196 \\
\hline $\mathrm{C}$ & -2.344399 & 2.043418 & -0.783553 & $\mathrm{H}$ & -1.716949 & 0.537312 & -2.233667 \\
\hline $\mathrm{C}$ & -2.253278 & 2.080833 & 1.649767 & $\mathrm{H}$ & -7.926101 & -2.873204 & -1.900723 \\
\hline $\mathrm{C}$ & -2.176831 & 3.432401 & -0.788644 & $\mathrm{H}$ & -7.170871 & -2.386809 & -3.420585 \\
\hline $\mathrm{C}$ & -2.121276 & 3.472197 & 1.599375 & $\mathrm{H}$ & -6.756545 & -3.942716 & -2.696688 \\
\hline $\mathrm{C}$ & -2.075002 & 4.171772 & 0.391623 & $\mathrm{H}$ & -3.064712 & 0.687605 & 3.128714 \\
\hline $\mathrm{C}$ & -5.475422 & 0.568706 & 0.837156 & $\mathrm{H}$ & -1.329183 & 0.929524 & 3.247728 \\
\hline $\mathrm{C}$ & -2.081598 & -2.540122 & -1.473678 & $\mathrm{H}$ & -2.433271 & 2.195574 & 3.797399 \\
\hline $\mathrm{C}$ & -2.447811 & 1.347242 & -2.127332 & $\mathrm{H}$ & -2.934771 & 6.15821 & 0.424099 \\
\hline $\mathrm{C}$ & -6.987755 & -2.897277 & -2.465209 & $\mathrm{H}$ & -1.358624 & 6.045516 & 1.210369 \\
\hline $\mathrm{C}$ & -2.27387 & 1.437108 & 3.023991 & $\mathrm{H}$ & -1.474928 & 6.023339 & -0.559484 \\
\hline $\mathrm{C}$ & -1.949305 & 5.676486 & 0.364774 & $\mathrm{H}$ & 3.858372 & -2.741532 & -1.263323 \\
\hline $\mathrm{C}$ & 3.776096 & -1.74094 & -1.700273 & $\mathrm{H}$ & 2.716825 & -1.502454 & -1.808588 \\
\hline $\mathrm{B}$ & -2.546968 & -0.24491 & 0.473671 & $\mathrm{H}$ & 4.231962 & -1.757749 & -2.703649 \\
\hline $\mathrm{N}$ & 4.393134 & -0.753799 & -0.818984 & & & & \\
\hline
\end{tabular}

Table S9. Coordinates $(\AA)$ for the optimized structure (B3LYP, 6-31G*) of 3.

\begin{tabular}{|c|c|c|c|c|r|r|r|}
\hline atom & $\mathrm{X}$ & $\mathrm{Y}$ & $\mathrm{Z}$ & atom & \multicolumn{1}{c|}{$\mathrm{X}$} & \multicolumn{1}{c|}{$\mathrm{Y}$} & \multicolumn{1}{c|}{$\mathrm{Z}$} \\
\hline $\mathrm{C}$ & 5.81756 & 0.273934 & -1.296978 & $\mathrm{H}$ & 2.526041 & 1.81172 & 0.017508 \\
\hline $\mathrm{C}$ & 4.871968 & -0.565327 & -0.689837 & $\mathrm{H}$ & 4.178945 & 3.25172 & -1.116255 \\
\hline $\mathrm{C}$ & 3.658512 & -0.004929 & -0.201092 & $\mathrm{H}$ & 6.324932 & 2.26649 & -1.926811 \\
\hline $\mathrm{C}$ & 3.435309 & 1.371528 & -0.381506 & $\mathrm{H}$ & 3.157889 & -1.720443 & 1.00046 \\
\hline $\mathrm{C}$ & 4.374114 & 2.188785 & -1.004428 & $\mathrm{H}$ & 0.963236 & 0.228982 & 0.118899 \\
\hline $\mathrm{C}$ & 5.575809 & 1.638677 & -1.451094 & $\mathrm{H}$ & 1.943461 & -2.497907 & 2.521349 \\
\hline $\mathrm{C}$ & 2.71002 & -0.843096 & 0.539032 & $\mathrm{H}$ & -2.919378 & -1.966482 & 1.838734 \\
\hline $\mathrm{C}$ & 1.385803 & -0.606813 & 0.666666 & $\mathrm{H}$ & 0.376816 & -3.90799 & 3.776021 \\
\hline $\mathrm{C}$ & 0.425784 & -1.39221 & 1.453731 & $\mathrm{H}$ & -2.085028 & -3.65241 & 3.433872 \\
\hline $\mathrm{C}$ & -0.981561 & -1.208792 & 1.280151 & $\mathrm{H}$ & -6.315426 & 0.349045 & -0.654482 \\
\hline $\mathrm{C}$ & 0.877122 & -2.369804 & 2.362705 & $\mathrm{H}$ & -4.128198 & -2.705421 & -2.705029 \\
\hline $\mathrm{C}$ & -1.847985 & -2.068423 & 1.9903 & $\mathrm{H}$ & -0.30441 & 3.936837 & -1.725809 \\
\hline $\mathrm{C}$ & -0.007971 & -3.169693 & 3.076756 & $\mathrm{H}$ & -0.224306 & 3.928488 & 2.552833 \\
\hline $\mathrm{C}$ & -1.384163 & -3.025373 & 2.889102 & $\mathrm{H}$ & -4.028762 & 1.076267 & 1.840705 \\
\hline $\mathrm{C}$ & -3.007376 & -0.481096 & -0.355564 & $\mathrm{H}$ & -3.760653 & 2.220172 & 0.530874 \\
\hline $\mathrm{C}$ & -4.233073 & 0.2088 & -0.150483 & $\mathrm{H}$ & -5.402683 & 1.680867 & 0.91321 \\
\hline $\mathrm{C}$ & -2.999376 & -1.55624 & -1.284303 & $\mathrm{H}$ & -0.850364 & -1.754674 & -1.654787 \\
\hline $\mathrm{C}$ & -5.383227 & -0.180552 & -0.844082 & $\mathrm{H}$ & -1.561367 & -3.108163 & -0.788972 \\
\hline $\mathrm{C}$ & -4.163975 & -1.891509 & -1.982623 & $\mathrm{H}$ & -1.86215 & -2.914365 & -2.521011 \\
\hline $\mathrm{C}$ & -5.370687 & -1.220917 & -1.776075 & $\mathrm{H}$ & -2.278858 & 1.267316 & -2.300741 \\
\hline $\mathrm{C}$ & -1.162144 & 1.413698 & 0.425918 & $\mathrm{H}$ & -0.890203 & 2.151511 & -2.951508 \\
\hline $\mathrm{C}$ & -0.959783 & 2.116689 & -0.793681 & $\mathrm{H}$ & -0.678118 & 0.537348 & -2.273416 \\
\hline $\mathrm{C}$ & -0.874347 & 2.098067 & 1.639789 & $\mathrm{H}$ & -7.517174 & -1.462324 & -1.945129 \\
\hline $\mathrm{C}$ & -0.472768 & 3.42836 & -0.778149 & $\mathrm{H}$ & -6.730135 & -0.952488 & -3.440071 \\
\hline
\end{tabular}




\begin{tabular}{|c|c|c|c|c|r|r|r|}
\hline $\mathrm{C}$ & -0.421158 & 3.420489 & 1.610125 & $\mathrm{H}$ & -6.578296 & -2.626652 & -2.897756 \\
\hline $\mathrm{C}$ & -0.20611 & 4.106513 & 0.412596 & $\mathrm{H}$ & -1.987327 & 0.89582 & 3.089927 \\
\hline $\mathrm{C}$ & -4.360225 & 1.355959 & 0.833661 & $\mathrm{H}$ & -0.241778 & 0.757837 & 3.231054 \\
\hline $\mathrm{C}$ & -1.752115 & -2.37056 & -1.576808 & $\mathrm{H}$ & -1.055344 & 2.225279 & 3.786592 \\
\hline $\mathrm{C}$ & -1.216963 & 1.483684 & -2.147985 & $\mathrm{H}$ & -0.571604 & 6.236711 & 0.538791 \\
\hline $\mathrm{C}$ & -6.613522 & -1.588147 & -2.551779 & $\mathrm{H}$ & 0.973567 & 5.732353 & 1.225472 \\
\hline $\mathrm{C}$ & -1.052678 & 1.458784 & 3.004549 & $\mathrm{H}$ & 0.760911 & 5.798049 & -0.53415 \\
\hline $\mathrm{C}$ & 0.268143 & 5.540463 & 0.409301 & $\mathrm{H}$ & 4.326061 & -3.856302 & -1.014947 \\
\hline $\mathrm{C}$ & 4.267083 & -2.824401 & -1.379791 & $\mathrm{H}$ & 3.224182 & -2.505463 & -1.338195 \\
\hline $\mathrm{C}$ & 6.484188 & -2.404723 & -0.519335 & $\mathrm{H}$ & 4.593915 & -2.811621 & -2.435109 \\
\hline $\mathrm{B}$ & -1.689078 & -0.082181 & 0.43011 & $\mathrm{H}$ & 6.966185 & -2.397376 & -1.514377 \\
\hline $\mathrm{N}$ & 5.097664 & -1.963102 & -0.534249 & $\mathrm{H}$ & 7.069322 & -1.772675 & 0.15429 \\
\hline $\mathrm{H}$ & 6.746379 & -0.14441 & -1.670687 & $\mathrm{H}$ & 6.520229 & -3.433961 & -0.144886 \\
\hline
\end{tabular}

Table S10. Coordinates $(\AA)$ for the optimized structure (B3LYP, 6-31G*) of 4.

\begin{tabular}{|c|c|c|c|c|c|c|c|}
\hline atom & $\mathrm{X}$ & $\mathrm{Y}$ & $\mathrm{Z}$ & atom & $\mathrm{X}$ & $\mathrm{Y}$ & Z \\
\hline $\mathrm{C}$ & 5.873086 & -0.318186 & -1.429367 & $\mathrm{H}$ & 3.015942 & 1.61422 & 0.32072 \\
\hline $\mathrm{C}$ & 4.86018 & -1.039451 & -0.775534 & $\mathrm{H}$ & 4.781986 & 2.864686 & -0.884381 \\
\hline $\mathrm{C}$ & 3.808981 & -0.328849 & -0.125427 & $\mathrm{H}$ & 6.64085 & 1.608697 & -1.979717 \\
\hline $\mathrm{C}$ & 3.805451 & 1.073158 & -0.193355 & $\mathrm{H}$ & 3.120705 & -1.995096 & 1.084266 \\
\hline $\mathrm{C}$ & 4.804438 & 1.779112 & -0.859473 & $\mathrm{H}$ & 1.171539 & 0.220278 & 0.277048 \\
\hline $\mathrm{C}$ & 5.844884 & 1.075139 & -1.466237 & $\mathrm{H}$ & 1.936352 & -2.420791 & 2.812293 \\
\hline $\mathrm{C}$ & 2.787843 & -1.06139 & 0.63096 & $\mathrm{H}$ & -2.882243 & -1.760385 & 1.944225 \\
\hline $\mathrm{C}$ & 1.507423 & -0.665818 & 0.806395 & $\mathrm{H}$ & 0.277778 & -3.688263 & 4.110237 \\
\hline $\mathrm{C}$ & 0.491999 & -1.347455 & 1.620464 & $\mathrm{H}$ & -2.160222 & -3.363362 & 3.674451 \\
\hline $\mathrm{C}$ & -0.90051 & -1.119453 & 1.394229 & $\mathrm{H}$ & -6.073273 & 0.462341 & -0.936165 \\
\hline $\mathrm{C}$ & 0.879885 & -2.274234 & 2.608168 & $\mathrm{H}$ & -3.897615 & -2.822158 & -2.608957 \\
\hline $\mathrm{C}$ & -1.820228 & -1.892899 & 2.134316 & $\mathrm{H}$ & 0.096845 & 3.792665 & -1.890399 \\
\hline $\mathrm{C}$ & -0.056533 & -2.988795 & 3.34797 & $\mathrm{H}$ & -0.058863 & 4.064431 & 2.377557 \\
\hline $\mathrm{C}$ & -1.419639 & -2.804104 & 3.108783 & $\mathrm{H}$ & -3.861822 & 1.337995 & 1.602235 \\
\hline $\mathrm{C}$ & -2.818891 & -0.448148 & -0.388996 & $\mathrm{H}$ & -3.543349 & 2.355447 & 0.203333 \\
\hline $\mathrm{C}$ & -4.026039 & 0.296817 & -0.309046 & $\mathrm{H}$ & -5.202093 & 1.871757 & 0.585925 \\
\hline $\mathrm{C}$ & -2.802678 & -1.596245 & -1.227673 & $\mathrm{H}$ & -0.646438 & -1.890244 & -1.465404 \\
\hline $\mathrm{C}$ & -5.152962 & -0.111539 & -1.030849 & $\mathrm{H}$ & -1.447726 & -3.144608 & -0.531355 \\
\hline $\mathrm{C}$ & -3.940682 & -1.950116 & -1.958175 & $\mathrm{H}$ & -1.653076 & -3.086813 & -2.287341 \\
\hline $\mathrm{C}$ & -5.13151 & -1.22547 & -1.871978 & $\mathrm{H}$ & -1.91306 & 1.121692 & -2.399471 \\
\hline $\mathrm{C}$ & -0.956244 & 1.442075 & 0.367011 & $\mathrm{H}$ & -0.479306 & 1.946292 & -3.030774 \\
\hline $\mathrm{C}$ & -0.664564 & 2.057249 & -0.882189 & $\mathrm{H}$ & -0.324956 & 0.380275 & -2.234639 \\
\hline $\mathrm{C}$ & -0.7133 & 2.195204 & 1.548671 & $\mathrm{H}$ & -7.270655 & -1.27432 & -2.209204 \\
\hline $\mathrm{C}$ & -0.141479 & 3.353423 & -0.923324 & $\mathrm{H}$ & -6.310178 & -1.180312 & -3.68816 \\
\hline $\mathrm{C}$ & -0.22025 & 3.501244 & 1.459849 & $\mathrm{H}$ & -6.409463 & -2.705347 & -2.805391 \\
\hline $\mathrm{C}$ & 0.078608 & 4.101512 & 0.235283 & $\mathrm{H}$ & -1.939218 & 1.124668 & 3.011571 \\
\hline $\mathrm{C}$ & -4.162604 & 1.528628 & 0.565737 & $\mathrm{H}$ & -0.209204 & 0.938889 & 3.251225 \\
\hline $\mathrm{C}$ & -1.571714 & -2.470007 & -1.38603 & $\mathrm{H}$ & -0.996254 & 2.464853 & 3.671241 \\
\hline $\mathrm{C}$ & -0.858287 & 1.337789 & -2.203472 & $\mathrm{H}$ & -0.232176 & 6.237327 & 0.066571 \\
\hline $\mathrm{C}$ & -6.344475 & -1.618692 & -2.68145 & $\mathrm{H}$ & 1.152206 & 5.789543 & 1.066875 \\
\hline $\mathrm{C}$ & -0.981877 & 1.65038 & 2.939519 & $\mathrm{H}$ & 1.251353 & 5.66499 & -0.699623 \\
\hline $\mathrm{C}$ & 0.593655 & 5.519474 & 0.164202 & $\mathrm{H}$ & 5.787583 & -2.994992 & -2.587458 \\
\hline $\mathrm{C}$ & 5.818101 & -3.208411 & -1.506047 & $\mathrm{H}$ & 6.837654 & -3.018974 & -1.152466 \\
\hline $\mathrm{B}$ & -1.530972 & -0.033391 & 0.436676 & $\mathrm{H}$ & 5.606929 & -4.270645 & -1.356222 \\
\hline $\mathrm{N}$ & 4.864437 & -2.435009 & -0.732901 & $\mathrm{H}$ & 3.937329 & -2.837706 & -0.717385 \\
\hline $\mathrm{H}$ & 6.679533 & -0.846664 & -1.925955 & & & & \\
\hline
\end{tabular}




\section{References:}

[1] M. J. Frisch, G. W. Trucks, H. B. Schlegel, G. E. Scuseria, M. A. Robb, J. R. Cheeseman, G. Scalmani, V. Barone, B. Mennucci, G. A. H. Petersson, M. Nakatsuji, X. Caricato, H. P. F. Li, A. Hratchian, J. Izmaylov, G. Bloino, J. L. Zheng, M. Sonnenberg, M. Hada, K. Ehara, R. Toyota, J. Fukuda, M. Hasegawa, T. Ishida, Y. Nakajima, O. Honda, H. Kitao, T. Nakai, J. A. Vreven, J. E. Montgomery Jr., F. Peralta, M. Ogliaro, J. J. Bearpark, E. Heyd, K. N. Brothers, V. N. Kudin, T. Staroverov, R. Keith, J. Kobayashi, K. Normand, A. Raghavachari, J. C. Rendell, S. S. Burant, J. Iyengar, M. Tomasi, N. Cossi, J. M. Rega, M. Millam, J. E. Klene, J. B. Knox, V. Cross, C. Bakken, J. Adamo, R. Jaramillo, R. E. Gomperts, O. Stratmann, A. J. Yazyev, R. Austin, C. Cammi, J. W. Pomelli, R. L. Ochterski, K. Martin, V. G. Morokuma, G. A. Zakrzewski, P. Voth, J. J. Salvador, S. Dannenberg, A. D. Dapprich, O. Daniels, J. B. Farkas, J. V. Foresman, J. Ortiz, J. Cioslowski, D. J. Fox, Gaussian 09 Revision C.01, 2010.

[2] (a) C. Lee, W. Yang, R. G. Parr, Phys. Rev. B, 1988, 37, 785-789; (b) A. D. Becke, J. Chem. Phys. 1993, 98, 1372-1377; (c) A. D. Becke, J. Chem. Phys. 1993, 98, 56485652.

[3] (a) R. Ditchfield, W. J. Hehre, J. A. Pople, J. Chem. Phys. 1971, 54, 724-728; (b) A. D. McLean, G. S. Chandler, J. Chem. Phys. 1980, 72, 5639-5648. 


\section{Electrochemical Measurements}
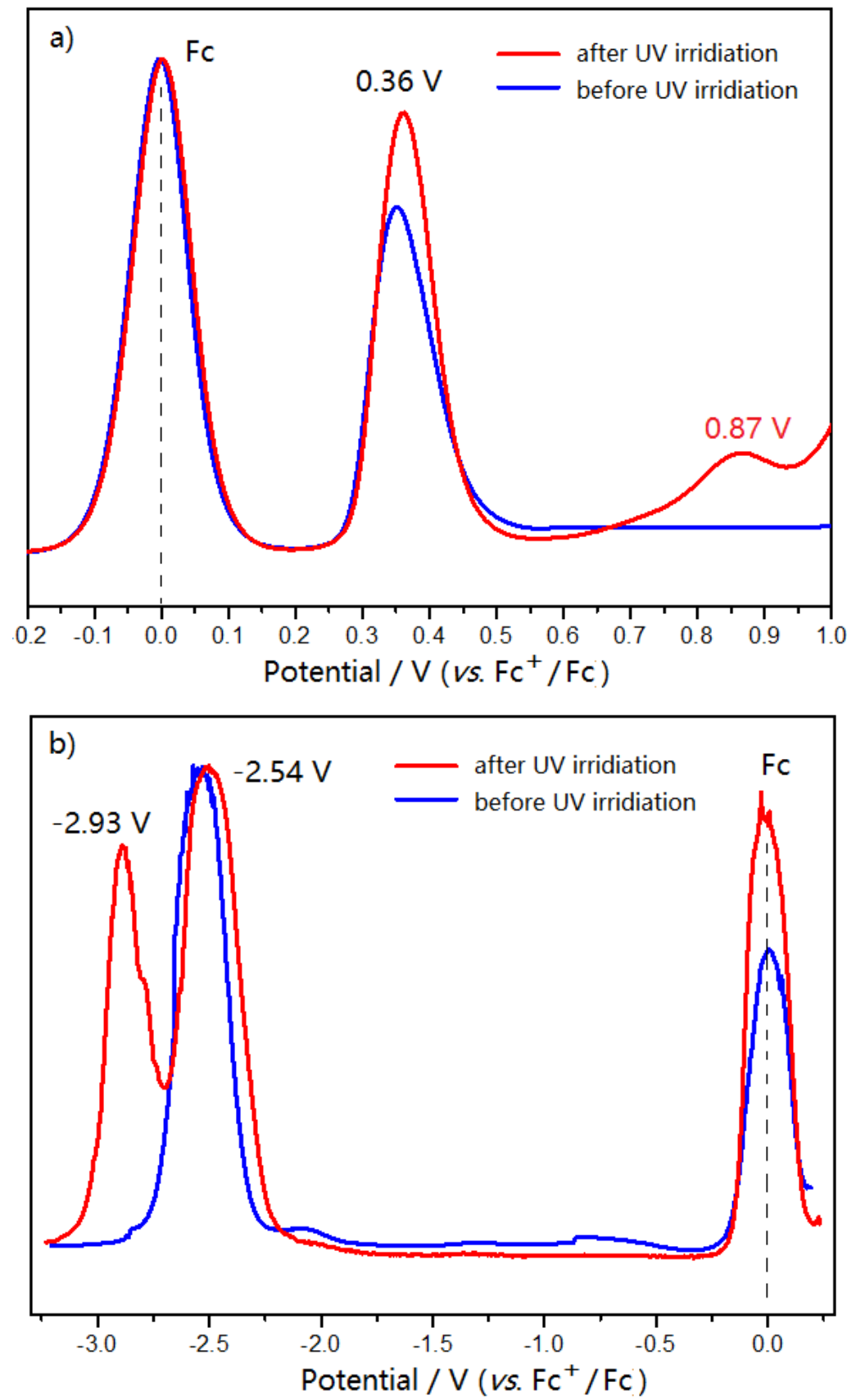

Figure S43. DVP electrochemistry of 4 before and after UV irradiation: a) oxidation in $\mathrm{CH}_{2} \mathrm{Cl}_{2}$ and $\left.b\right)$ reduction in THF with $\left[\mathrm{Bu}_{4} \mathrm{~N}\right]\left[\mathrm{PF}_{6}\right](c=0.1 \mathrm{M})$ as the electrolyte), $v=$ $100 \mathrm{mV} / \mathrm{s}$. 